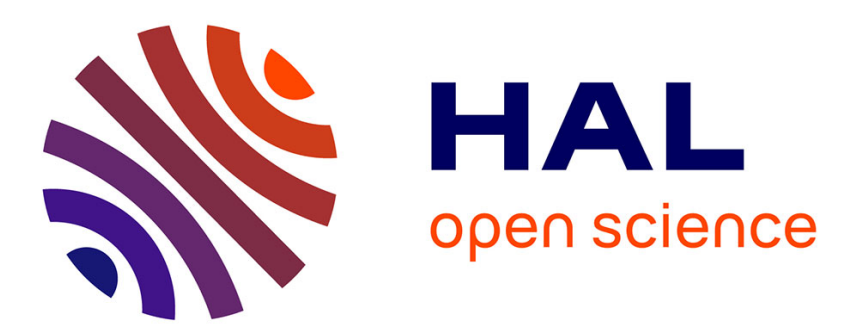

\title{
Finite volume method for general multifluid flows governed by the interface Stokes problem Stella Krell
}

\section{To cite this version:}

Stella Krell. Finite volume method for general multifluid flows governed by the interface Stokes problem. Mathematical Models and Methods in Applied Sciences, 2012, 22 (5), pp.1150025. 10.1142/S0218202511500254 . hal-00473783v2

\section{HAL Id: hal-00473783 \\ https://hal.science/hal-00473783v2}

Submitted on 25 Sep 2010

HAL is a multi-disciplinary open access archive for the deposit and dissemination of scientific research documents, whether they are published or not. The documents may come from teaching and research institutions in France or abroad, or from public or private research centers.
L'archive ouverte pluridisciplinaire HAL, est destinée au dépôt et à la diffusion de documents scientifiques de niveau recherche, publiés ou non, émanant des établissements d'enseignement et de recherche français ou étrangers, des laboratoires publics ou privés. 


\title{
FINITE VOLUME METHOD FOR GENERAL MULTIFLUID FLOWS GOVERNED BY THE INTERFACE STOKES PROBLEM
}

\author{
STELLA KRELL*
}

\begin{abstract}
We study the approximation of solutions to the steady Stokes problem with a discontinuous viscosity coefficient (interface Stokes problem) in the 2D "Discrete Duality Finite Volume" (DDFV) framework. In order to take into account the discontinuities of the viscosity and to prevent consistency defect in the scheme, we propose to modify the definition of the numerical fluxes on the edges of the mesh where the discontinuity occurs. We first show how to design our modified scheme, called m-DDFV, and we analyze its well-posedness and its convergence properties. Finally, we provide numerical results which confirm that the m-DDFV scheme significantly improves the convergence rate of the usual DDFV method for Stokes problems.
\end{abstract}

Key words. Interface Stokes problem, discontinuous coefficients, DDFV methods.

\section{Introduction.}

1.1. Interface Stokes model. In this paper, we are concerned with the finite volume approximation of solutions to the steady interface Stokes problem with homogeneous Dirichlet boundary conditions: Find a velocity $\mathbf{u}: \Omega \rightarrow \mathbb{R}^{2}$ and a pressure $p: \Omega \rightarrow \mathbb{R}$ such that:

$$
\operatorname{div}(-\varphi(\mathbf{u}, p))=\mathbf{f}, \quad \operatorname{div}(\mathbf{u})=0, \text { in } \Omega, \quad \mathbf{u}=0, \text { on } \partial \Omega, \quad \int_{\Omega} p(x) \mathrm{d} x=0 .
$$

where $\Omega$ is a polygonal connected open bounded subset of $\mathbb{R}^{2}$, the total stress tensor is denoted by $\varphi(\mathbf{u}, p)=2 \eta(x) \mathrm{Du}-p \mathrm{Id}$, with $\mathrm{Du}=\frac{1}{2}\left(\nabla \mathbf{u}+{ }^{t} \nabla \mathbf{u}\right), \mathbf{f}$ is a function in $\left(L^{2}(\Omega)\right)^{2}$ and the viscosity $\eta \in L^{\infty}(\Omega)$ satisfies:

$$
0<\underline{\mathrm{C}}_{\eta} \leq \eta(x) \leq \overline{\mathrm{C}}_{\eta}, \text { for a.e. } x \in \Omega,
$$

where $\underline{\mathrm{C}}_{\eta}$ and $\overline{\mathrm{C}}_{\eta}$ are two positive constants. For simplicity we will only consider here the case of homogeneous Dirichlet boundary conditions, we emphasize the fact that our framework naturally allows to take into account non-homogeneous Dirichlet boundary conditions.

REMARK 1.1. Here we note the stress tensor by $\varphi$ instead of the usual notation $\sigma$ since, in the finite volume framework, $\sigma$ traditionally denotes an edge of the mesh.

The existence and uniqueness of a solution $(\mathbf{u}, p) \in\left(H_{0}^{1}(\Omega)\right)^{2} \times L^{2}(\Omega)$ of (1.1) is classical using the Lax-Milgram Theorem and the Nečas Lemma (see for instance [6, 16, 27, 25]).

In particular, this study allows to take into account a viscosity constant per sub-domains $\Omega_{1}, \Omega_{2}$ such that $\Omega_{1} \cap \Omega_{2}=\emptyset$ and $\bar{\Omega}=\overline{\Omega_{1}} \cup \overline{\Omega_{2}}$. On the interface $\Gamma=\partial \Omega_{1} \cap \partial \Omega_{2}$ between the sub-domains, we have the following condition

$$
[\mathbf{u}]_{\left.\right|_{\Gamma}}=0 \quad \text { and } \quad[\varphi(\mathbf{u}, p) \overrightarrow{\mathbf{n}}]_{\left.\right|_{\Gamma}}=0 \text {, on } \Gamma,
$$

where $\overrightarrow{\mathbf{n}}$ is an unit normal vector to $\Gamma$ oriented from $\Omega_{1}$ to $\Omega_{2}$ and $[a]_{\left.\right|_{\Gamma}}=\left(a_{\left.\right|_{\Omega_{1}}}-a_{\left.\right|_{\Omega_{2}}}\right){ }_{\left.\right|_{\Gamma}}$ denotes the jump of $a$ across $\Gamma$. Since the viscosity is discontinuous across the interface $\Gamma$, the pressure may have jumps. More precisely, we have $[p]_{\left.\right|_{\Gamma}}=[2 \eta \operatorname{Du} \overrightarrow{\mathbf{n}} \cdot \overrightarrow{\mathbf{n}}]_{\left.\right|_{\Gamma}}$ on $\Gamma$ see [20]. Thus, our scheme must consider the possible jumps of the pressure and of the velocity gradient. The corresponding regularity of the solution is then (for more details see [24])

$$
\begin{aligned}
& \mathbf{u} \in\left\{\mathbf{v} \in\left(H_{0}^{1}(\Omega)\right)^{2}, \quad \mathbf{v}_{\left.\right|_{\Omega_{i}}} \in\left(H^{2}\left(\Omega_{i}\right)\right)^{2} \text { for } i=1,2\right\}, \text { for the velocity, } \\
& p \in\left\{q \in L^{2}(\Omega), \quad q_{\left.\right|_{\Omega_{i}}} \in H^{1}\left(\Omega_{i}\right), \text { for } i=1,2\right\}, \text { for the pressure. }
\end{aligned}
$$

${ }^{*}$ Université de Provence, Laboratoire d'Analyse, Topologie et Probabilités, 39 rue F. Joliot-Curie, 13453 Marseille Cedex 13, France (krellecmi.univ-mrs.fr) 
In many numerical simulations, two phase flows are modeled by a single set of conservation laws for the whole computational domain. Such an approach leads to Navier-Stokes equations with discontinuous density and viscosity coefficients. Thus the Stokes equations with discontinuous viscosity (1.1) can be considered as a reasonable first step for the study of highly viscous two phase flows.

1.2. The DDFV method. Different methods of gradient reconstruction for cell-centered finite volume methods have been proposed since the last ten years to handle anisotropic heterogeneous scalar diffusion problem on distorted meshes. In all cases, the crucial feature is that the summation-by-parts procedure permits to reconstruct a whole two dimensional discrete gradient, starting from two point finite differences. Many of them have been compared in the benchmark of the FVCA5 conference [18], for scalar diffusion problems, see also $[8,10,11,14,19]$ for more details.

We consider here the class of schemes called "Discrete Duality Finite Volume" (DDFV for short). The DDFV method has been first introduced and studied in [10,19] to approximate the solution of the Laplace equation on a large class of 2D meshes including non-conformal and distorted meshes, without "orthogonality" assumptions required by classical finite volume methods. Basically, it consists in defining a full discrete gradient from finite differences in two independent directions. This discrete gradient (see Definition 2.1) is located around the edges of the mesh and his dual operator, the discrete divergence (see Definition 2.2) on the centers and the vertices of the mesh.

All the notation used in this introduction are defined in Section 2.

1.2.1. The DDFV method for the Stokes problem. Finite volume approximation of Stokes problems is a current research topic, we refer to [9, 12, 15, 2, 3] for the description and the analysis of the main available schemes up to now. All these works deal with a constant viscosity on the whole domain. We propose here a staggered method: the discrete unknowns (the components of the velocity and the pressure) are located on different nodes. The most celebrated staggered scheme is the MAC scheme [17, 23] on cartesian grids. Actually, for a cartesian grid and constant viscosity, the scheme we propose here is equivalent (except possibly on the boundary) to two uncoupled MAC schemes written on two different staggered meshes.

The first reason why the DDFV method is considered here, is the large class of 2D general meshes we can use. The second one is: since the viscous part of the momentum conservation law is not a Laplace operator, we have to address the problem of the reconstruction of the full velocity gradient and its symmetric part on the whole domain. The DDFV strategy for the Stokes problem is the following: the approximate velocity $\mathbf{u}^{\mathcal{T}}$ is defined on the centers and the vertices of the mesh, and the approximate pressure $p^{\mathfrak{D}}$ on the edges of the mesh, that is where the discrete velocity gradient exists. Remark that the edges are naturally associated to a family of quadrangles called diamond cells (see Fig. 2.2(a)).

In a previous work [21], we propose the following construction of the scheme in the case of smooth viscosity. We integrate the momentum conservation law of the problem (1.1) on the interior center cells $\mathfrak{M}$ and the interior vertex cells $\mathfrak{M}^{*}$. The mass conservation equation is directly approached on the diamond cells. The velocity is imposed to be equal to zero on the boundary of the domain, which is denoted by $\mathbf{u}^{\mathcal{T}} \in \mathbb{E}_{0}$ (see (2.3)). Finally, the integral of the pressure is imposed to be equal to zero. Unfortunately, the corresponding scheme is only proved to be well-posed for particular classes of mesh see [9]. Indeed, the well-posedness result relies on a discrete inf-sup condition, which is still an open problem for general meshes. To overcome this difficulty, we propose in [21] to add in the mass conservation equation a stabilization term $-\lambda h_{\mathfrak{D}}^{2} \Delta^{\mathfrak{D}} p^{\mathfrak{D}}$, corresponding to a finite volume approximation of the Laplace operator (see Definition 2.9), inspired by the well-known Brezzi-Pitkäranta method 
in the finite element framework [7]. The stabilized DDFV scheme can then be written as follows:

$$
\begin{array}{ll}
\text { Find } \mathbf{u}^{\mathcal{T}} \in \mathbb{E}_{0} \text { and } p^{\mathfrak{D}} \in \mathbb{R}^{\mathfrak{D}} \text { such that, } \\
\operatorname{div}_{\mathfrak{M}}\left(-2 \eta^{\mathfrak{D}} \mathrm{D}^{\mathfrak{D}} \mathbf{u}^{\mathcal{T}}+p^{\mathfrak{D}} \mathrm{Id}\right)=\mathbf{f}^{\mathfrak{M}}, & \operatorname{div}^{\mathfrak{M}}{ }^{*}\left(-2 \eta^{\mathfrak{D}} \mathrm{D}^{\mathfrak{D}} \mathbf{u}^{\mathcal{T}}+p^{\mathfrak{D}} \mathrm{Id}\right)=\mathbf{f}^{\mathfrak{M}}, \\
\operatorname{div}^{\mathfrak{D}}\left(\mathbf{u}^{\mathcal{T}}\right)-\lambda h_{\mathfrak{D}}^{2} \Delta^{\mathfrak{D}} p^{\mathfrak{D}}=0, & \sum_{\mathcal{D} \in \mathfrak{D}} m_{\mathcal{D}} p^{\mathcal{D}}=0,
\end{array}
$$

where $\lambda>0$ is given and is a stabilisation parameter. This stabilized DDFV scheme is then proved to be well-posed for general 2D meshes. Furthermore, we showed the convergence of such schemes and error estimates in the case where the viscosity and the exact solution are assumed to be smooth enough (see [21]). We proved the first order convergence of the scheme (1.4) in the $L^{2}(\Omega)$-norm for the velocity gradient, as well as for the velocity and for the pressure. These results have been extended to the 3D case in [22]. In the case where $\eta$ presents discontinuities, our numerical results in [21] show that the scheme is still convergent but the error analysis is no more valid and, actually, we numerically observe a loss of accuracy of the method in that case.

1.2.2. Consideration on the discontinuities of the viscosity. Even for scalar diffusion problems, it is known that such discontinuities in the coefficients imply a consistency defect in the numerical fluxes of usual finite volume schemes. It is needed to modify the scheme in order to take into account the jumps of the coefficients of the problem and then to recover the optimal first order convergence rate. As in the scalar case [5], we need to introduce a modified gradient operator (see Definition 2.5) and finally define a modified approximate viscous stress tensor $\mathrm{D}_{\mathfrak{D}}^{\eta, \mathcal{N}} \mathbf{u}^{\mathcal{T}}$ (see Definition 2.7) on each diamond cell. We derive a modified DDFV scheme, referred to as m-DDFV, that consists in replacing $\eta^{\mathfrak{D}} \mathrm{D}^{\mathfrak{D}} \mathbf{u}^{\mathcal{T}}$ (resp. $\left.-\lambda h_{\mathfrak{D}}^{2} \Delta^{\mathfrak{D}} p^{\mathfrak{D}}\right)$ by $\mathrm{D}_{\mathfrak{D}}^{\eta, \mathcal{N}} \mathbf{u}^{\mathcal{T}}$, (resp. $\left.-\lambda h_{\mathfrak{D}}^{2} \Delta^{\mathfrak{D}}\left(p^{\mathfrak{D}}, \mathrm{D}^{\mathfrak{D}} \mathbf{u}^{\mathcal{T}}\right)\right)$ as follows:

$$
\begin{array}{ll}
\text { Find } \mathbf{u}^{\mathcal{T}} \in \mathbb{E}_{0} \text { and } p^{\mathfrak{D}} \in \mathbb{R}^{\mathfrak{D}} \text { such that, } \\
\operatorname{div}^{\mathfrak{M}}\left(-2 \mathrm{D}_{\mathfrak{D}}^{\eta, \mathcal{N}} \mathbf{u}^{\mathcal{T}}+p^{\mathfrak{D}} \mathrm{Id}\right)=\mathbf{f}^{\mathfrak{M}} \\
\operatorname{div}^{\mathfrak{D}}\left(\mathbf{u}^{\mathcal{T}}\right)-\lambda h_{\mathfrak{D}}^{2} \Delta^{\mathfrak{D}}\left(p^{\mathfrak{D}}, \mathrm{D}^{\mathfrak{D}} \mathbf{u}^{\mathcal{T}}\right)=0, \quad \operatorname{div}^{\mathfrak{M}}{ }^{*}\left(-2 \mathrm{D}_{\mathfrak{D}}^{\eta, \mathcal{N}} \mathbf{u}^{\mathcal{T}}+p^{\mathfrak{D}} \mathrm{Id}\right)=\mathbf{f}^{\mathfrak{M}},
\end{array}
$$

Note that this m-DDFV scheme has the same number of unknowns as the standard DDFV scheme (1.4). The aim of this work is first to explain the derivation of this new scheme. Then we show an existence and uniqueness result which relies on a discrete Korn inequality on the modified discrete operators (see Theorem 4.2) which is not just an extension of the one proved in [21]. We finally provide a first order error estimate of the scheme (1.5) in the $L^{2}(\Omega)$-norm for the velocity gradient and for the pressure. Furthermore, we numerically observe the real benefit of this construction. We want to emphasize that, despite quite intricate notations and construction, the implementation of m-DDFV schemes is in fact easy. It is essentially the same as that for the DDFV scheme (see Section 2.4.5) and the computational costs of the two methods are almost the same.

1.3. Outline. This paper is organized as follows. In Section 2, we recall the DDFV framework for the finite volume approximation of Stokes problems on unstructured 2D grids and we introduce the modified discrete operators (see Section 2.4). Then, we describe the m-DDFV stabilized scheme in Section 3. In Section 4, we present the main results of discrete functional analysis necessary for the theoretical study of the finite volume method. These results include properties of discrete operators proved in [21] but also properties of the modified discrete operators, including an appropriate discrete Korn inequality (see Theorem 4.2). We prove the stability and well-posedness of the scheme in Section 5. Then, in Section 6, we prove error estimates (see Theorem 6.1). Finally theoretical error estimates are illustrated with numerical results, in Section 7. 


\section{The DDFV framework.}

\subsection{The meshes and notation.}

The meshes. We recall here the main notation and definitions taken from [1]. A DDFV mesh $\mathcal{T}$ is constituted by a primal mesh $\mathfrak{M} \cup \partial \mathfrak{M}$ and a dual mesh $\mathfrak{M}^{*} \cup \partial \mathfrak{M}^{*}$. An example for square locally refined primal mesh is given in Fig. 2.1.
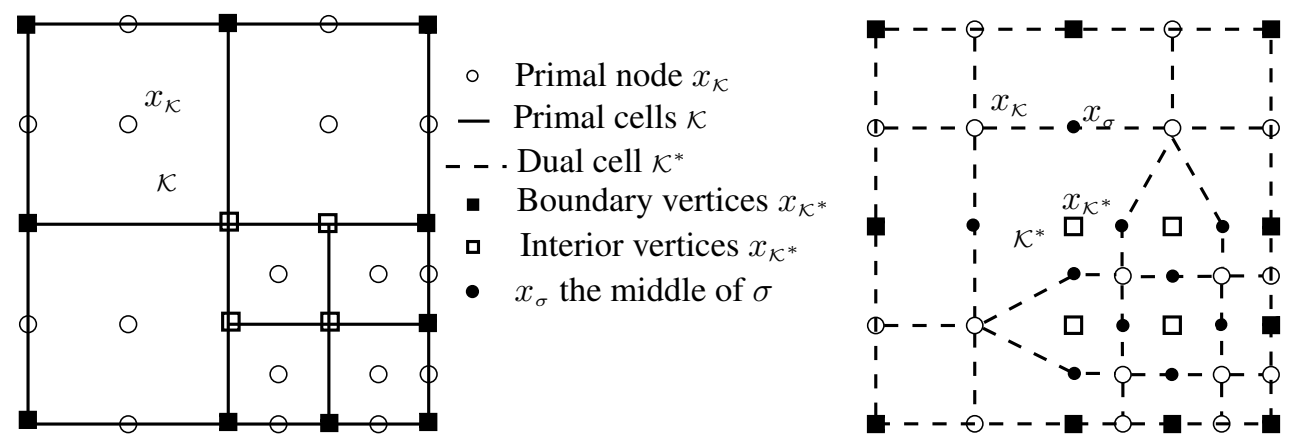

FIG. 2.1. The mesh $\mathcal{T}$. (Left) The primal mesh $\mathfrak{M} \cup \partial \mathfrak{M}$. (Right) The dual mesh $\mathfrak{M}^{*} \cup \partial \mathfrak{M}^{*}$.

The interior primal mesh $\mathfrak{M}$ is a set of disjoint open polygonal control volumes $\mathcal{K} \subset \Omega$ such that $\cup \overline{\mathcal{K}}=\bar{\Omega}$. We denote by $\partial \mathfrak{M}$ the set of edges of the control volumes in $\mathfrak{M}$ included in $\partial \Omega$, which we consider as degenerate control volumes. To each control volume and degenerate control volume $\mathcal{K} \in \mathfrak{M} \cup \partial \mathfrak{M}$, we associate a point $x_{\mathcal{K}} \in \mathcal{K}$. For each degenerate control volume $\mathcal{K} \in \partial \mathfrak{M}$, we choose the point $x_{\mathcal{K}}$ to be the midpoint of the control volume $\mathcal{K}$. This family of points is denoted by $X=\left\{x_{\mathcal{K}}, \mathcal{K} \in \mathfrak{M} \cup \partial \mathfrak{M}\right\}$.

For all control volumes $\mathcal{K}$ and $\mathcal{L}$, we assume that $\partial \mathcal{K} \cap \partial \mathcal{L}$ is either empty or a common vertex or an edge of the primal mesh denoted by $\sigma=\mathcal{K} \mid \mathcal{L}$. We note by $\mathcal{E}$ the set of such edges. We also note $\sigma^{*}$ the segment $\left[x_{\mathcal{K}}, x_{\mathcal{L}}\right]$ and $\mathcal{E}^{*}$ the set of such segments. To each edge $\sigma \in \mathcal{E}$, we associate a point $x_{\sigma}$ such that $x_{\sigma}$ belongs to the interior of $\sigma$. We introduce, for each edge $\sigma \in \mathcal{E}$, two different angles: $\alpha_{\mathcal{K}}$ the angle between $\overrightarrow{x_{\mathcal{K}} x_{\sigma}}$ and $\sigma, \alpha_{\mathcal{L}}$ the angle between $\overrightarrow{x_{\mathcal{L}} x_{\sigma}}$ and $\sigma$, (see Fig. 2.2(a)).

Let $X^{*}$ denote the set of the vertices of the primal control volumes in $\mathfrak{M}$ that we split into $X^{*}=X_{\text {int }}^{*} \cup X_{\text {ext }}^{*}$ where $X_{\text {int }}^{*} \cap \partial \Omega=\emptyset$ and $X_{\text {ext }}^{*} \subset \partial \Omega$. With any point $x_{\mathcal{K}^{*}} \in X_{\text {int }}^{*}$ (resp. $x_{\mathcal{K}^{*}} \in X_{\text {ext }}^{*}$ ), we associate the polygon $\mathcal{K}^{*} \in \mathfrak{M}^{*}$ (resp. $\mathcal{K}^{*} \in \partial \mathfrak{M}^{*}$ ) whose sides are $\left\{\left[x_{\mathcal{K}}, x_{\sigma}\right]\right.$ such that $\left.x_{\mathcal{K}} \in X, x_{\mathcal{K}^{*}} \in \overline{\mathcal{K}} \cap \bar{\sigma}, \mathcal{K} \in \mathfrak{M}, \sigma \in \mathcal{E}\right\}$ (resp. $\left\{\left[x_{\mathcal{K}^{*}}, x_{\sigma}\right]\right.$ such that $\sigma \in$ $\partial \mathfrak{M}$ and $\left.x_{\mathcal{K}^{*}} \in \bar{\sigma}\right\} \cup\left\{\left[x_{\mathcal{K}}, x_{\sigma}\right]\right.$ such that $\left.x_{\mathcal{K}} \in X, x_{\mathcal{K}^{*}} \in \overline{\mathcal{K}} \cap \bar{\sigma}, \mathcal{K} \in \mathfrak{M}, \sigma \in \mathcal{E}\right\}$ ) sorted with respect to the clockwise order of the corresponding control volumes. This defines the set $\mathfrak{M}^{*} \cup \partial \mathfrak{M}^{*}$ of dual control volumes.

CRITERION 2.1. For each $\sigma \in \mathcal{E}$, we usually choose for $x_{\sigma}$ the middle point of the edge $\sigma$. In that case, dual cells are called barycentric dual cells. For each $\sigma \in \mathcal{E}$, we can define the two angles $\alpha_{\mathcal{K}}$ and $\alpha_{\mathcal{L}}$ as shown in Fig. 2.2(a). We specify a criterion $\epsilon_{0}>0$ such that if the angles are too close $\left|\alpha_{\mathcal{K}}-\alpha_{\mathcal{L}}\right|<\epsilon_{0}$, then $x_{\sigma}$ is finally chosen to be the intersection of the primal edge $\sigma$ and the segment $\sigma^{*}$.

We modify some dual cells in order to have either the same angles like for the direct dual mesh (see $[1,5,10,21])$ or the angles distant from $\epsilon_{0}$. This technical assumption plays a role in Definition 2.5 of the modified discrete gradient, in the discrete Korn inequality (see Theorem 4.2) and in the consistency errors analysis (see Lemma 6.4 and Corollary 6.1). The reason is that those three results rely on the estimate given in Lemma 2.2, which can be seen to blow up as soon as the angles $\alpha_{\mathcal{K}}$ and $\alpha_{\mathcal{L}}$ are too close but distinct. 
Given the primal and dual control volumes, we define the diamond cells $\mathcal{D}_{\sigma, \sigma^{*}}$ being the quadrangles whose diagonals are a primal edge $\sigma=\mathcal{K} \mid \mathcal{L}=\left[x_{\mathcal{K}^{*}}, x_{\mathcal{L}^{*}}\right]$ and a corresponding segment $\sigma^{*}=\left[x_{\mathcal{K}}, x_{\mathcal{L}}\right]$, (see Fig. 2.2(a)). Note that the diamond cells are not necessarily convex. If $\sigma \in \mathcal{E} \cap \partial \bar{\Omega}$, the quadrangle $\mathcal{D}_{\sigma, \sigma^{*}}$ degenerates into a triangle. The set of the diamond cells is denoted by $\mathfrak{D}$ and we have $\bar{\Omega}=\underset{\mathcal{D} \in \mathfrak{D}}{\cup} \overline{\mathcal{D}}$.

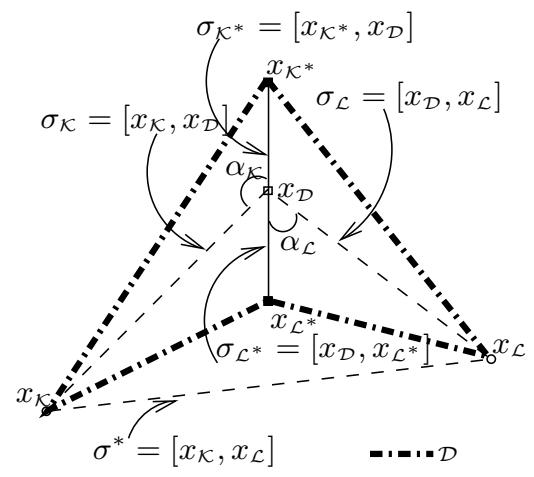

(a) Notation in the diamond cell.

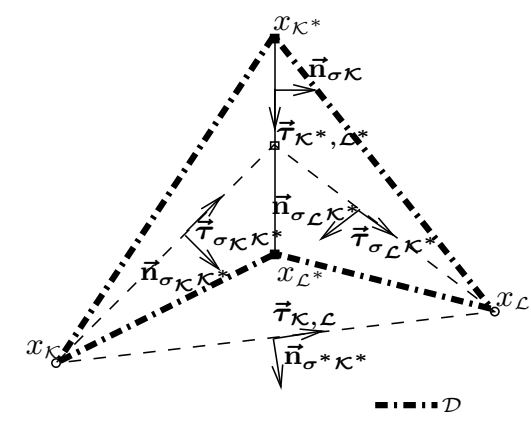

(b) Direct orthonormal basis on the diamond cell.

FIG. 2.2. Diamond cells.

Notation. We recall here the main notation taken from [21]. For any primal control volume $\mathcal{K} \in \mathfrak{M} \cup \partial \mathfrak{M}$, we note $m_{\mathcal{K}}$ its Lebesgue measure, $d_{\mathcal{K}}$ its diameter, $\mathcal{E}_{\mathcal{K}}$ the set of its edges (if $\mathcal{K} \in \mathfrak{M}$ ), or the one-element set $\{\mathcal{K}\}$ if $\mathcal{K} \in \partial \mathfrak{M}, \mathfrak{D}_{\mathcal{K}}=\left\{\mathcal{D}_{\sigma, \sigma^{*}} \in \mathfrak{D}, \sigma \in \mathcal{E}_{\mathcal{K}}\right\}$, $B_{\mathcal{K}}:=B\left(x_{\mathcal{K}}, \rho_{\mathcal{K}}\right) \cap \partial \Omega \subset \overline{\mathcal{K}}$ the open ball of radius $\rho_{\mathcal{K}}>0$ for $\mathcal{K} \in \partial \mathfrak{M}, m_{B_{\mathcal{K}}}$ its measure, the value $\rho_{\mathcal{K}}$ is chosen such that the inclusion is verified. We will also use corresponding dual notation for any dual cells $\mathcal{K}^{*} \in \mathfrak{M}^{*} \cup \partial \mathfrak{M}^{*}: m_{\mathcal{K}^{*}}, \mathcal{E}_{\mathcal{K}^{*}}, \mathfrak{D}_{\mathcal{K}^{*}}, d_{\mathcal{K}^{*}}, B_{\mathcal{K}^{*}}, m_{B_{\mathcal{K}^{*}}}, \rho_{\mathcal{K}^{*}}$.

For a diamond cell $\mathcal{D}=\mathcal{D}_{\sigma, \sigma^{*}}$ whose vertices are $\left(x_{\mathcal{K}}, x_{\mathcal{K}^{*}}, x_{\mathcal{L}}, x_{\mathcal{L}^{*}}\right)$, we note $x_{\mathcal{D}}=x_{\sigma}$ the center of the diamond cell $\mathcal{D}, h_{\mathcal{D}}$ its diameter, $m_{\sigma}$ the length of the primal edge $\sigma, m_{\sigma^{*}}$ the length of $\sigma^{*}$ and $m_{\mathcal{D}}$ its measure. We introduce for each diamond cell in Fig. 2.2(b) the two direct orthonormal basis $\left(\overrightarrow{\boldsymbol{\tau}}_{\mathcal{K}^{*}, \mathcal{L}^{*}}, \overrightarrow{\mathbf{n}}_{\sigma \mathcal{K}}\right)$ and $\left(\overrightarrow{\mathbf{n}}_{\sigma^{*} \mathcal{K}^{*}}, \overrightarrow{\boldsymbol{\tau}}_{\mathcal{K}, \mathcal{L}}\right)$, where $\overrightarrow{\mathbf{n}}_{\sigma \mathcal{K}}$ the unit vector normal to $\sigma$ oriented from $x_{\mathcal{K}}$ to $x_{\mathcal{L}}, \overrightarrow{\mathbf{n}}_{\sigma^{*} \mathcal{K}^{*}}$ the unit vector normal to $\sigma^{*}$ oriented from $x_{\mathcal{K}^{*}}$ to $x_{\mathcal{L}^{*}}, \overrightarrow{\boldsymbol{\tau}}_{\mathcal{K}, \mathcal{L}}$ the unit vector parallel to $\sigma^{*}$ oriented from $x_{\mathcal{K}}$ to $x_{\mathcal{L}}$ and $\overrightarrow{\boldsymbol{\tau}}_{\mathcal{K}^{*}, \mathcal{L}^{*}}$ the unit vector parallel to $\sigma$ oriented from $x_{\mathcal{K}^{*}}$ to $x_{\mathcal{L}^{*}}$. We also note for each diamond cell $\mathfrak{s}$ its sides (for

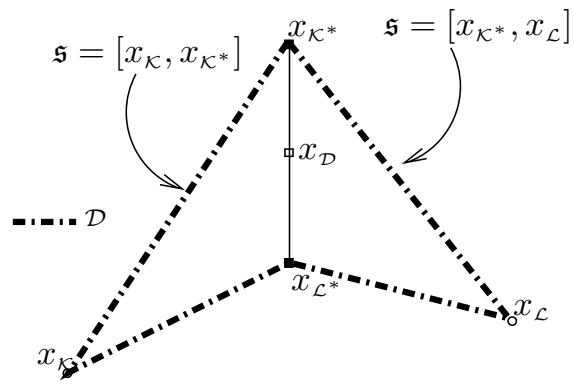

(a) A diamond and its sides.

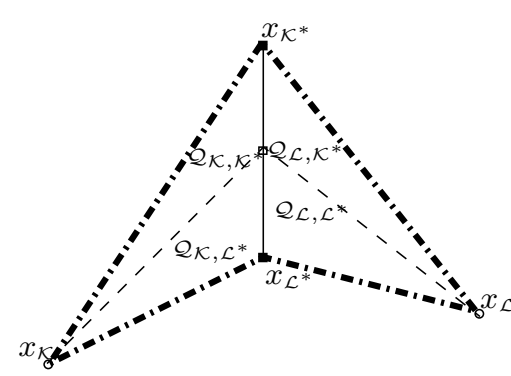

(b) Quarter diamond cells.

FIG. 2.3. Diamond cells.

example $\mathfrak{s}=\left[x_{\mathcal{K}}, x_{\mathcal{K}^{*}}\right]$ see Fig. 2.3(a)), $\mathcal{E}_{\mathcal{D}}=\{\mathfrak{s}, \mathfrak{s} \subset \partial \mathcal{D}$ and $\mathfrak{s} \not \subset \partial \Omega\}$ the set of interior 
sides of $\mathcal{D}, m_{\mathfrak{s}}$ the length of a diamond side $\mathfrak{s}, \overrightarrow{\mathbf{n}}_{\mathfrak{s} \mathcal{D}}$ the unit vector normal to $\mathfrak{s}=\mathcal{D} \mid \mathcal{D}^{\prime}$ oriented from $\mathcal{D}$ to $\mathcal{D}^{\prime}$ and $\mathfrak{S}=\left\{\mathfrak{s} \in \mathcal{E}_{\mathcal{D}}, \forall \mathcal{D} \in \mathfrak{D}\right\}$ the set of interior sides of all diamond cells in $\mathfrak{D}$.

Since we use here the barycentric dual cells, we introduce other notation in comparison to the notation in [21]. For a diamond cell $\mathcal{D}$, we note $\sigma_{\mathcal{K}}\left(\right.$ resp. $\left.\sigma_{\mathcal{L}}\right)$ the segment $\left[x_{\mathcal{K}}, x_{\mathcal{D}}\right]$ (resp. $\left.\left[x_{\mathcal{D}}, x_{\mathcal{L}}\right]\right), \sigma_{\mathcal{K}^{*}}\left(\right.$ resp. $\left.\sigma_{\mathcal{L}^{*}}\right)$ the segment $\left[x_{\mathcal{K}^{*}}, x_{\mathcal{D}}\right]$ (resp. $\left.\left[x_{\mathcal{D}}, x_{\mathcal{L}^{*}}\right]\right), m_{\varsigma}$ the length of $\varsigma$ and $x_{\varsigma}$ the middle point of the segment $\varsigma$ for each $\varsigma \in\left\{\sigma_{\mathcal{K}}, \sigma_{\mathcal{L}}, \sigma_{\mathcal{K}^{*}}, \sigma_{\mathcal{L}^{*}}\right\}$. We introduce in Fig. 2.2(b) the two other direct orthonormal basis $\left(\overrightarrow{\mathbf{n}}_{\sigma_{\mathcal{K}} \mathcal{K}^{*}}, \vec{\tau}_{\sigma_{\mathcal{K}} \mathcal{K}^{*}}\right)$ and $\left(\overrightarrow{\mathbf{n}}_{\sigma_{\mathcal{L}} \mathcal{K}^{*}}, \vec{\tau}_{\sigma_{\mathcal{L}^{\mathcal{K}}}}\right)$, where $\overrightarrow{\mathbf{n}}_{\sigma_{\mathcal{K}} \mathcal{K}^{*}}$ the unit vector normal to $\sigma_{\mathcal{K}}$ oriented from $x_{\mathcal{K}^{*}}$ to $x_{\mathcal{L}^{*}}, \overrightarrow{\mathbf{n}}_{\sigma_{\mathcal{L}} \mathcal{K}^{*}}$ the unit vector normal to $\sigma_{\mathcal{L}}$ oriented from $x_{\mathcal{K}^{*}}$ to $x_{\mathcal{L}^{*}}, \vec{\tau}_{\sigma_{\mathcal{K}} \mathcal{K}^{*}}$ the unit vector parallel to $\sigma_{\mathcal{K}}$ oriented from $x_{\mathcal{K}}$ to $x_{\mathcal{D}}$ and $\vec{\tau}_{\sigma_{\mathcal{L}} \mathcal{K}^{*}}$ the unit vector parallel to $\sigma_{\mathcal{L}}$ oriented from $x_{\mathcal{D}}$ to $x_{\mathcal{L}}$. Remark that we have $m_{\sigma^{*}} \overrightarrow{\mathbf{n}}_{\sigma^{*} \mathcal{K}^{*}}=m_{\sigma_{\mathcal{K}}} \overrightarrow{\mathbf{n}}_{\sigma_{\mathcal{K}} \mathcal{K}^{*}}+m_{\sigma_{\mathcal{L}}} \overrightarrow{\mathbf{n}}_{\sigma_{\mathcal{L}} \mathcal{K}^{*}}$, for any $\mathcal{D} \in \mathfrak{D}$.

We distinguish the interior diamond cells and the boundary diamond cells: $\mathfrak{D}_{\text {ext }}=\{\mathcal{D} \in$ $\mathfrak{D}, \mathcal{D} \cap \partial \Omega \neq \emptyset\}, \mathfrak{D}_{\text {int }}=\mathfrak{D} \backslash \mathfrak{D}_{\text {ext }}$. For all $\mathcal{D} \in \mathfrak{D}_{\text {ext }}$, we define the length between $x_{\mathcal{K}^{*}}$ (resp. $x_{\mathcal{L}^{*}}$ ) and $x_{\mathcal{L}}$ by $d_{\mathcal{K}^{*}, \mathcal{L}}\left(\right.$ resp. $d_{\mathcal{L}^{*}, \mathcal{L}}$ ). Thus, for all $\mathcal{D} \in \mathfrak{D}_{\text {ext }}$, we have $m_{\sigma_{\mathcal{K}^{*}}}=d_{\mathcal{K}^{*}, \mathcal{L}}$ and $m_{\sigma_{\mathcal{L}^{*}}}=d_{\mathcal{L}^{*}, \mathcal{L}}$.

To each diamond cell $\mathcal{D} \in \mathfrak{D}$, we associate quarter diamond cells as follows $\mathcal{Q}_{p, d}=$ $\overline{\mathcal{D}} \cap \bar{p} \cap \bar{d}$, such that $p \cap \mathcal{D} \neq \emptyset$ and $d \cap \mathcal{D} \neq \emptyset$, for $p \in\{\mathcal{K}, \mathcal{L}\}$ and $d \in\left\{\mathcal{K}^{*}, \mathcal{L}^{*}\right\}$, as shown in Fig. 2.3(b). If $\mathcal{D} \in \mathfrak{D}_{\text {int }}$, we have $\overline{\mathcal{D}}=\overline{\mathcal{Q}_{\mathcal{K}, \mathcal{K}^{*}}} \cup \overline{\mathcal{Q}_{\mathcal{K}, \mathcal{L}^{*}}} \cup \overline{\mathcal{Q}_{\mathcal{L}, \mathcal{K}^{*}}} \cup \overline{\mathcal{Q}_{\mathcal{L}, \mathcal{L}^{*}}}$ and if $\mathcal{D} \in \mathfrak{D}_{\text {ext }}$, we have $\overline{\mathcal{D}}=\overline{\mathcal{Q}_{\mathcal{K}, \mathcal{K}^{*}}} \cup \overline{\mathcal{Q}_{\mathcal{K}, \mathcal{L}^{*}}}$. The set of the quarter diamonds in the domain is denoted by $\mathfrak{Q}=\underset{\mathcal{D} \in \mathfrak{D}}{\cup} \mathfrak{Q}_{\mathcal{D}}$. For $\mathcal{Q} \in \mathfrak{Q}$, we note by $m_{\mathcal{Q}}$ its measure and $h_{\mathcal{Q}}$ its diameter. We also define the set $\mathcal{E}_{\mathcal{Q}}=\left\{\sigma_{\mathcal{K}}, \sigma_{\mathcal{L}}, \sigma_{\mathcal{K}^{*}}, \sigma_{\mathcal{L}^{*}}\right\}$, for all $\mathcal{Q} \in \mathfrak{Q}$. Remark that $m_{\mathcal{Q}}$ is, for instance for $\mathcal{Q}=\mathcal{Q}_{\mathcal{K}, \mathcal{K}^{*}}$, equal to $\frac{1}{2} \sin \left(\alpha_{\mathcal{K}}\right) m_{\sigma_{\mathcal{K}}} m_{\sigma_{\mathcal{K}^{*}}}$.

ASSUMPTION 2.1. An important assumption for our analysis is that each DDFV mesh $\mathcal{T}$ is conforming with respect to the discontinuities of the viscosity. We assume that the viscosity $\eta$ is Lipschitz continuous on each quarter diamond cell: there exists $\mathrm{C}_{\eta}>0$ such that:

$$
\left|\eta(x)-\eta\left(x^{\prime}\right)\right| \leq \mathrm{C}_{\eta}\left|x-x^{\prime}\right|, \quad \forall x, x^{\prime} \in \overline{\mathcal{Q}}, \quad \text { for all } \mathcal{Q} \in \mathfrak{Q} \text {. }
$$

We note $\eta_{\mathcal{Q}}=\frac{1}{m_{\mathcal{Q}}} \int_{\mathcal{Q}} \eta(s) \mathrm{d} s$, for all $\mathcal{Q} \in \mathfrak{Q}$. We always have $\underline{\mathrm{C}}_{\eta} \leq \eta_{\mathcal{Q}} \leq \overline{\mathrm{C}}_{\eta}$, for all $\mathcal{Q} \in \mathfrak{Q}$.

This assumption imposes to know where the discontinuity occurs before building the mesh of the domain. Of course, in real non-stationary situations this is not possible and it would be interesting to extend our analysis to the case of immersed interfaces. However, one can see that the present work can be adapted to the case of the linear elasticity equations for which this assumption seems much more realistic.

We denote by $\mathcal{M}_{m, n}(\mathbb{R})$ the set of real $m \times n$ matrices (we note $\mathcal{M}_{n}(\mathbb{R})$ when $m=$ $n$ ). In the sequel, $\|\cdot\|_{2}$ stands for the natural $L^{2}(\Omega)$-norm when we consider scalar valued and vector valued functions and for the Frobenius norm when we consider matrix valued functions:

$$
\|\xi\|_{2}^{2}=\int_{\Omega}\|\xi(x)\|_{\mathcal{F}}^{2} \mathrm{~d} x, \text { with }\|\xi\|_{\mathcal{F}}^{2}=(\xi: \xi), \quad \forall \xi \in L^{2}\left(\Omega, \mathcal{M}_{2}(\mathbb{R})\right)
$$

where $(\xi: \widetilde{\xi})=\sum_{1 \leq i, j \leq 2} \xi_{i, j} \widetilde{\xi}_{i, j}=\operatorname{Tr}\left({ }^{t} \xi \widetilde{\xi}\right), \quad \forall \xi, \widetilde{\xi} \in \mathcal{M}_{2}(\mathbb{R})$.

REMARK 2.1. The matrix norm $\|\cdot\|_{\mathcal{F}}$ satisfies $\left\|\frac{A+{ }^{t} A}{2}\right\|\left\|_{\mathcal{F}} \leq\right\| A \|_{\mathcal{F}}$, for all $A \in \mathcal{M}_{2}(\mathbb{R})$.

Mesh regularity measurement. Let $\operatorname{size}(\mathcal{T})$ be the maximum of the diameters of the diamond cells in $\mathfrak{D}$. To measure how flat the diamond cells can be, we note $\alpha_{\mathcal{T}}$ the unique real in $\left.] 0, \frac{\pi}{2}\right]$ such that $\sin \left(\alpha_{\mathcal{T}}\right):=\min _{\mathcal{D} \in \mathfrak{D}}\left(\left|\sin \left(\alpha_{\mathcal{K}}\right)\right|,\left|\sin \left(\alpha_{\mathcal{L}}\right)\right|\right)$. We introduce a positive number $\operatorname{reg}(\mathcal{T})$ that quantifies the regularity of a given mesh and is useful to perform the convergence 
analysis of finite volume schemes:

$$
\operatorname{reg}(\mathcal{T}):=\max \left(\frac{1}{\sin \left(\alpha_{\mathcal{T}}\right)}, \max _{\mathcal{D} \in \mathcal{D}} \max _{\mathcal{Q} \in \mathfrak{Q}_{\mathcal{D}}} \frac{h_{\mathcal{D}}}{\min _{\sigma \in \partial \mathcal{Q}} m_{\sigma}}, \max _{\mathcal{K} \in \mathfrak{M}_{\mathcal{D}} \in \mathcal{D} \mathcal{K}} \frac{d_{\mathcal{K}}}{h_{\mathcal{D}}}, \max _{\mathcal{K}^{*} \in \mathfrak{M}^{*} \cup \partial \mathfrak{M}^{*} \mathcal{D} \in \mathfrak{P}_{\mathcal{K}^{*}}} \frac{d_{\mathcal{K}^{*}}}{h_{\mathcal{D}}}\right) .
$$

The number $\operatorname{reg}(\mathcal{T})$ should be uniformly bounded when $\operatorname{size}(\mathcal{T}) \rightarrow 0$ for the convergence to hold. For instance, there exists a constant $C$ depending on $\operatorname{reg}(\mathcal{T})$ such that

$$
\frac{h_{\mathcal{D}}}{\sqrt{m_{\mathcal{D}}}} \leq \frac{h_{\mathcal{D}}}{\sqrt{m_{\mathcal{Q}}}} \leq C, \forall \mathcal{Q} \in \mathfrak{Q}_{\mathcal{D}}, \quad h_{\mathcal{D}} \leq C \min \left(m_{\sigma}, m_{\sigma^{*}}\right), \forall \mathcal{D}_{\sigma, \sigma^{*}} \in \mathfrak{D} .
$$

2.2. Unknowns and discrete projections. The DDFV method associates to any primal cell $\mathcal{K} \in \mathfrak{M} \cup \partial \mathfrak{M}$ an unknown value $\mathbf{u}_{\mathcal{K}} \in \mathbb{R}^{2}$ for the velocity, to any dual cell $\mathcal{K}^{*} \in$ $\mathfrak{M}^{*} \cup \partial \mathfrak{M}^{*}$ an unknown value $\mathbf{u}_{\mathcal{K}^{*}} \in \mathbb{R}^{2}$ for the velocity and to any diamond cell $\mathcal{D} \in \mathfrak{D}$ an unknown value $p^{\mathcal{D}} \in \mathbb{R}$ for the pressure. These unknowns are collected in the families :

$$
\mathbf{u}^{\mathcal{T}}=\left(\left(\mathbf{u}_{\mathcal{K}}\right)_{\mathcal{K} \in(\mathfrak{M} \cup \partial \mathfrak{M})},\left(\mathbf{u}_{\mathcal{K}^{*}}\right)_{\mathcal{K}^{*} \in\left(\mathfrak{M}^{*} \cup \partial \mathfrak{M}^{*}\right)}\right) \in\left(\mathbb{R}^{2}\right)^{\mathcal{T}}, \quad p^{\mathfrak{D}}=\left(\left(p^{\mathcal{D}}\right)_{\mathcal{D} \in \mathfrak{D}}\right) \in \mathbb{R}^{\mathfrak{D}}
$$

We specify a discrete subset of $\left(\mathbb{R}^{2}\right)^{\mathcal{T}}$ needed to take into account the Dirichlet boundary conditions:

$$
\mathbb{E}_{0}=\left\{\mathbf{v}^{\mathcal{T}} \in\left(\mathbb{R}^{2}\right)^{\mathcal{T}} \text { s. t. } \mathbf{v}_{\mathcal{K}}=0, \forall \mathcal{K} \in \partial \mathfrak{M} \text { and } \mathbf{v}_{\mathcal{K}^{*}}=0, \forall \mathcal{K}^{*} \in \partial \mathfrak{M}^{*}\right\} .
$$

We define an interior mean-value projection for any integrable vector function $\mathbf{v}$ on $\Omega$ :

$$
\widetilde{\mathbb{P}}_{\boldsymbol{m}}^{\mathfrak{m}} \mathbf{v}=\left(\left(\frac{1}{m_{\mathcal{K}}} \int_{\mathcal{K}} \mathbf{v}(x) \mathrm{d} x\right)_{\mathcal{K} \in \mathfrak{M}}\right), \widetilde{\mathbb{P}}_{\boldsymbol{m}}^{\mathfrak{M}^{*}} \mathbf{v}=\left(\left(\frac{1}{m_{\mathcal{K}^{*}}} \int_{\mathcal{K}^{*}} \mathbf{v}(x) \mathrm{d} x\right)_{\mathcal{K}^{*} \in \mathfrak{M}^{*}}\right) .
$$

We also note the mean-value projection for any integrable vector function $\mathbf{v}$ on $\bar{\Omega}$ as follows

$$
\mathbb{P}_{\boldsymbol{m}}^{\mathcal{I}} \mathbf{v}=\left(\widetilde{\mathbb{P}}_{\boldsymbol{m}}^{\mathfrak{m}} \mathbf{v},\left(\frac{1}{m_{B_{\mathcal{K}}}} \int_{B_{\mathcal{K}}} \mathbf{v}(x) \mathrm{d} x\right)_{\mathcal{K} \in \partial \mathfrak{M}}, \widetilde{\mathbb{P}}_{\boldsymbol{m}}^{\mathfrak{M}^{*}} \mathbf{v},\left(\frac{1}{m_{B_{\mathcal{K}^{*}}}} \int_{B_{\mathcal{K}^{*}}} \mathbf{v}(x) \mathrm{d} x\right)_{\mathcal{K}^{*} \in \partial \mathfrak{M}^{*}}\right)
$$

In particular, the mean-value projection $\mathbb{P}_{m}^{\mathcal{T}} \mathbf{v}$ is well defined for any vector field $\mathbf{v}$ lying in $\left(H^{1}(\Omega)\right)^{2}$.

2.3. Discrete operators. We recall the discrete operators introduced in [21].

DEFINITION 2.1 (Discrete gradient). We define a consistent approximation of the gradient operator $\nabla^{\mathfrak{D}}: \mathbf{u}^{\mathcal{T}} \in\left(\mathbb{R}^{2}\right)^{T^{\mathcal{T}}} \mapsto\left(\nabla^{\mathcal{D}} \mathbf{u}^{\mathcal{T}}\right)_{\mathcal{D} \in \mathfrak{D}} \in\left(\mathcal{M}_{2}(\mathbb{R})\right)^{\mathfrak{D}}$, as follows:

$$
\nabla^{\mathcal{D}} \mathbf{u}^{\mathcal{T}}=\frac{1}{\sin \left(\alpha_{\mathcal{D}}\right)}\left[\frac{\mathbf{u}_{\mathcal{L}}-\mathbf{u}_{\mathcal{K}}}{m_{\sigma^{*}}} \otimes \overrightarrow{\mathbf{n}}_{\sigma \mathcal{K}}+\frac{\mathbf{u}_{\mathcal{L}^{*}}-\mathbf{u}_{\mathcal{K}^{*}}}{m_{\sigma}} \otimes \overrightarrow{\mathbf{n}}_{\sigma^{*} \mathcal{K}^{*}}\right] .
$$

where $\otimes$ represents the tensor product.

DEFINITION 2.2 (Discrete divergence). We define a consistent approximation of the divergence operator applied to discrete tensor fields denoted by $\operatorname{div}^{\mathcal{T}}:\left(\mathcal{M}_{2}(\mathbb{R})\right)^{\mathfrak{D}} \mapsto\left(\mathbb{R}^{2}\right)^{\mathcal{T}}$ such that $\operatorname{div}^{\mathcal{T}} \xi^{\mathcal{D}}=\left(\operatorname{div}^{\mathfrak{M}} \xi^{\mathcal{D}}, \operatorname{div}^{\partial \mathfrak{M}} \xi^{\mathfrak{D}}, \operatorname{div}^{\mathfrak{M}{ }^{*}} \xi^{\mathcal{D}}, \operatorname{div}^{\partial \mathfrak{M}^{*}} \xi^{\mathfrak{D}}\right)$, for $\xi^{\mathcal{D}} \in\left(\mathcal{M}_{2}(\mathbb{R})\right)^{\mathfrak{D}}$, with $\operatorname{div}^{\mathfrak{M}} \xi^{\mathfrak{D}}=\left(\operatorname{div}^{\mathcal{\kappa}} \xi^{\mathfrak{D}}\right)_{\mathcal{K} \in \mathfrak{M}^{\prime}}, \operatorname{div}^{\partial \mathfrak{M}} \xi^{\mathcal{D}}=0, \operatorname{div}^{\mathfrak{M} *} \xi^{\mathfrak{D}}=\left(\operatorname{div}^{\mathcal{K}^{*}} \xi^{\mathfrak{D}}\right)_{\mathcal{K}^{*} \in \mathfrak{M}^{*}}, \operatorname{div}^{\partial \mathfrak{M}^{*}} \xi^{\mathcal{D}}=$ 


$$
\begin{aligned}
\left(\operatorname{div}^{\mathcal{K}^{*}} \xi^{\mathfrak{D}}\right)_{\mathcal{K}^{*} \in \partial \mathfrak{M} *} \text { and: } & \\
\operatorname{div}^{\mathcal{K}} \xi^{\mathfrak{D}} & =\frac{1}{m_{\mathcal{K}}} \sum_{\sigma \in \partial \mathcal{K}} m_{\sigma} \xi^{\mathcal{D}} \overrightarrow{\mathbf{n}}_{\sigma \mathcal{K}}, \forall \mathcal{K} \in \mathfrak{M}, \\
\operatorname{div}^{\mathcal{K}^{*}} \xi^{\mathfrak{D}} & =\frac{1}{m_{\mathcal{K}^{*}}} \sum_{\sigma * \partial \mathcal{K}^{*}} m_{\sigma^{*}} \xi^{\mathcal{D}} \overrightarrow{\mathbf{n}}_{\sigma^{*} \mathcal{K}^{*}}, \forall \mathcal{K}^{*} \in \mathfrak{M}^{*}, \\
\operatorname{div}^{\mathcal{K}^{*}} \xi^{\mathfrak{D}} & =\frac{1}{m_{\mathcal{K}^{*}}}\left(\sum_{\mathcal{D}_{\sigma, \sigma^{*}} \in \mathfrak{D}_{\mathcal{K}^{*}}} m_{\sigma^{*}} \xi^{\mathcal{D}} \overrightarrow{\mathbf{n}}_{\sigma^{*} \mathcal{K}^{*}}+\sum_{\mathcal{D}_{\sigma, \sigma^{*}} \in \mathfrak{D}_{\mathcal{K}^{*} \cap \mathfrak{D}_{e x t}}} d_{\mathcal{K}^{*}, \mathcal{L}} \xi^{\mathcal{D}} \overrightarrow{\mathbf{n}}_{\sigma \mathcal{K}}\right), \forall \mathcal{K}^{*} \in \partial \mathfrak{M}^{*} .
\end{aligned}
$$

Using the barycentric dual mesh, we also can write the discrete divergence like in [9]

$$
\operatorname{div}^{\mathcal{K}^{*}} \xi^{\mathfrak{D}}=\frac{1}{m_{\mathcal{K}^{*}}} \sum_{\sigma^{*} \in \partial \mathcal{K}^{*}}\left(m_{\sigma_{\mathcal{K}}} \xi^{\mathcal{D}} \overrightarrow{\mathbf{n}}_{\sigma_{\mathcal{K}} \mathcal{K}^{*}}+m_{\sigma_{\mathcal{L}}} \xi^{\mathcal{D}} \overrightarrow{\mathbf{n}}_{\sigma_{\mathcal{L}} \mathcal{K}^{*}}\right), \forall \mathcal{K}^{*} \in \mathfrak{M}^{*}
$$

Thanks to the discrete gradient we can define a discrete strain rate tensor and a discrete divergence of a vector field in $\left(\mathbb{R}^{2}\right)^{\mathcal{T}}$.

DEFINITION 2.3 (Discrete strain rate tensor). We define a discrete strain rate tensor of a vector field in $\left(\mathbb{R}^{2}\right)^{\mathcal{T}}, \mathrm{D}^{\mathfrak{D}}: \mathbf{u}^{\mathcal{T}} \in\left(\mathbb{R}^{2}\right)^{\mathcal{T}} \mapsto\left(\mathrm{D}^{\mathcal{D}} \mathbf{u}^{\mathcal{T}}\right)_{\mathcal{D} \in \mathfrak{D}} \in\left(\mathcal{M}_{2}(\mathbb{R})\right)^{\mathfrak{D}}$, with $\mathrm{D}^{\mathcal{D}} \mathbf{u}^{\mathcal{T}}=$ $\frac{\nabla^{\mathcal{D}} \mathbf{u}^{\mathcal{T}}+{ }^{t}\left(\nabla^{\mathcal{D}} \mathbf{u}^{\mathcal{T}}\right)}{2}$, for all $\mathcal{D} \in \mathfrak{D}$.

DEFINITION 2.4. We define a discrete divergence of a vector field in $\left(\mathbb{R}^{2}\right)^{\mathcal{T}}$, div ${ }^{\mathfrak{D}}$ : $\mathbf{u}^{\mathcal{T}} \in\left(\mathbb{R}^{2}\right)^{\mathcal{T}} \mapsto\left(\operatorname{div}^{\mathcal{D}} \mathbf{u}^{\mathcal{T}}\right)_{\mathcal{D} \in \mathcal{D}} \in \mathbb{R}^{\mathcal{D}}$, with $\operatorname{div}^{\mathcal{D}} \mathbf{u}^{\mathcal{T}}=\operatorname{Tr}\left(\nabla^{\mathcal{D}} \mathbf{u}^{\mathcal{T}}\right)$, for all $\mathcal{D} \in \mathfrak{D}$.

2.4. Local modification of the discrete strain rate tensor. The point we are concerned with in this paper is that the DDFV scheme (1.4) suffers from a loss of consistency in the case where $\eta$ presents discontinuities. More precisely, we present a way to recover the consistency of the fluxes even when $\eta$ jumps across the primal and dual edges of the mesh.

We observe that, at the continuous level, the normal component of the stress tensor $\varphi(\mathbf{u}, p)=2 \eta \mathrm{Du}-p \mathrm{Id}$ is continuous in a weak sense across all primal and dual edges of the mesh. For instance, we have

$$
\int_{\sigma_{\mathcal{K}^{*}}} \varphi_{\mid \overline{\mathcal{Q}}_{\mathcal{K}, \mathcal{K}^{*}}}(\mathbf{u}, p) \overrightarrow{\mathbf{n}}_{\sigma \mathcal{K}} \mathrm{d} s=\int_{\sigma_{\mathcal{K}^{*}}} \varphi_{\mid \overline{\mathcal{Q}}_{\mathcal{L}, \mathcal{K}^{*}}}(\mathbf{u}, p) \overrightarrow{\mathbf{n}}_{\sigma \mathcal{K}} \mathrm{d} s .
$$

We need to ensure an equivalent continuity property at the discrete level. We express a discrete stress tensor $\varphi_{\mathcal{Q}}$ as follows $\varphi_{\mathcal{Q}}=2 \eta_{\mathcal{Q}} \mathrm{D}_{\mathcal{Q}}^{\mathcal{N}} \mathbf{u}^{\mathcal{T}}-p^{\mathcal{Q}}$ Id on quarter diamond cells, (see Definition 2.8) thanks to additional unknowns $p^{\mathfrak{Q}_{\mathcal{D}}}=\left(p^{\mathcal{Q}}\right)_{\mathcal{Q} \in \mathfrak{Q}_{\mathcal{D}}}$ and a modified strain rate tensor $\mathrm{D}_{\mathcal{Q}}^{\mathcal{N}}$ (see Definition 2.6). The additional unknowns will be algebraically eliminated on each diamond cell (see Section 2.4.3). Thus the number of unknowns of the m-DDFV scheme is the same as for the DDFV scheme.

2.4.1. Scalar diffusion problems. We first recall the principle of the method proposed in [5] for scalar diffusion problems. The discrete gradient $\nabla^{\mathcal{D}} u^{\mathcal{T}}$ can be understood as the gradient of the unique affine function $\Pi_{\mathcal{D}} u^{\mathcal{T}}$ on $\mathcal{D}$ whose value at the middle of each side of the diamond $\mathcal{D}$ is the mean value between the two unknowns associated to the extremities of this segment (this construction is summed up in Fig. 2.4). The modified discrete gradient $\nabla_{\mathcal{Q}}^{\mathcal{N}} u^{\mathcal{T}}$ is chosen to be constant on all the quarter diamond cells $\mathcal{Q} \in \mathfrak{Q}$. It is the gradient of a function $\widetilde{\Pi}_{\mathcal{D}} u^{\mathcal{T}}$ which is affine on each $\mathcal{Q} \in \mathfrak{Q}_{\mathcal{D}}$, which coincides with $\Pi_{\mathcal{D}} u^{\mathcal{T}}$ in the middle of each side of $\mathcal{D}$ and which is continuous at each point $x_{\sigma_{\mathcal{K}}}, x_{\sigma_{\mathcal{L}}}, x_{\sigma_{\mathcal{K}^{*}}}, x_{\sigma_{\mathcal{L}^{*}}}$. The modified discrete gradient can be expressed as $\nabla^{\mathcal{D}} u^{\mathcal{T}}+B_{\mathcal{Q}} \delta^{\mathcal{D}}$ where $\delta^{\mathcal{D}}={ }^{t}\left(\delta_{\mathcal{K}}, \delta_{\mathcal{L}}, \delta_{\mathcal{K}^{*}}, \delta_{\mathcal{L}^{*}}\right) \in \mathbb{R}^{4}$ is a set of four unknowns that are $\widetilde{\Pi}_{\mathcal{D}} u^{\mathcal{T}}(y)-\Pi_{\mathcal{D}} u^{\mathcal{T}}(y)$ for each $y \in\left\{x_{\sigma_{\mathcal{K}}}, x_{\sigma_{\mathcal{L}}}, x_{\sigma_{\mathcal{K}^{*}}}, x_{\sigma_{\mathcal{L}^{*}}}\right\}$ 

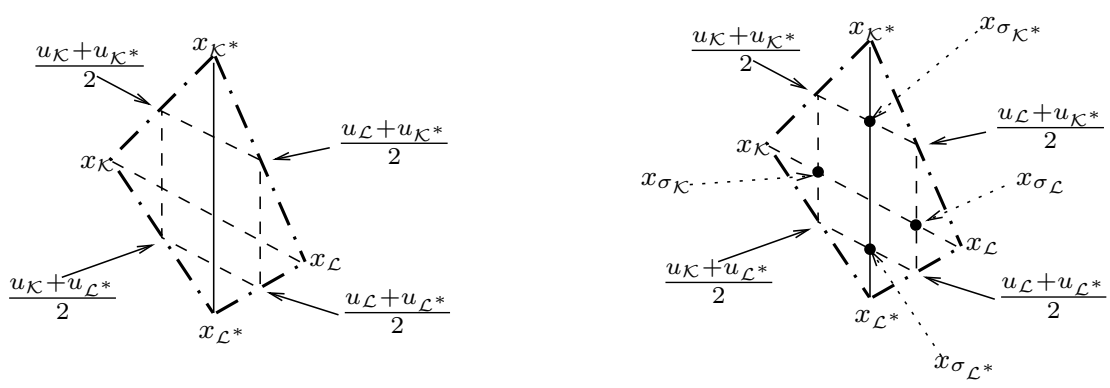

FIG. 2.4. The affine functions $\Pi_{\mathcal{D}} u^{\mathcal{T}}$ and $\widetilde{\Pi}_{\mathcal{D}} u^{\mathcal{T}}$ on $\mathcal{D}$.

and $\left(B_{\mathcal{Q}}\right)_{\mathcal{Q} \in \mathfrak{Q}_{\mathcal{D}}}$ is a family of matrices in $\mathcal{M}_{2,4}(\mathbb{R})$ which can be explicitly computed and depend on the geometry of $\mathcal{D}$. Remark that the modified discrete gradient depends on the artificial unknowns $\delta^{\mathcal{D}}$, which can be determined. This construction, valid for an interior diamond, can be extended to the case where $\mathcal{D} \in \mathfrak{D}_{\text {ext }}$ with $\delta^{\mathcal{D}}=\left(\delta_{\mathcal{K}}\right) \in \mathbb{R}$. If we note $n_{\mathcal{D}}=4$ if $\mathcal{D} \in \mathfrak{D}_{\text {int }}$ and $n_{\mathcal{D}}=1$ if $\mathcal{D} \in \mathfrak{D}_{\text {ext }}$, then that $\delta^{\mathcal{D}}$ is a vector in $\mathbb{R}^{n_{\mathcal{D}}}$.

2.4.2. Modified operators in the vector-valued case. We propose here to adapt the above framework to the vector case. We will now work with $\delta^{\mathcal{D}}={ }^{t}\left(\delta_{\mathcal{K}}, \delta_{\mathcal{L}}, \delta_{\mathcal{K}^{*}}, \delta_{\mathcal{L}^{*}}\right)$ lying in $\mathcal{M}_{n_{\mathcal{D}}, 2}$ instead of a vector in $\mathbb{R}^{n_{\mathcal{D}}}$ and the family of matrix $B_{\mathcal{Q}}$ is the same.

DEFINITION 2.5 (Discrete gradient on quarter diamonds). A discrete gradient of a vector field of $\left(\mathbb{R}^{2}\right)^{\mathcal{T}}, \nabla_{\mathfrak{Q}}^{\mathcal{N}}:\left(\mathbb{R}^{2}\right)^{\mathcal{T}} \rightarrow\left(\nabla_{\mathcal{Q}}^{\mathcal{N}} \mathbf{u}^{\mathcal{T}}\right)_{\mathcal{Q} \in \mathfrak{Q}} \in\left(\mathcal{M}_{2}(\mathbb{R})\right)^{\mathfrak{Q}}$, is a set of tensors defined by $\nabla_{\mathcal{Q}}^{\mathcal{N}} \mathbf{u}^{\mathcal{T}}=\nabla^{\mathcal{D}} \mathbf{u}^{\mathcal{T}}+{ }^{t} \delta^{\mathcal{D} t} B_{\mathcal{Q}}$, for any $\mathcal{D} \in \mathfrak{D}$ and for any $\mathcal{Q} \in \mathfrak{Q}_{\mathcal{D}}$, with $\delta^{\mathcal{D}} \in\left(\mathcal{M}_{n_{\mathcal{D}}, 2}(\mathbb{R})\right)^{\mathfrak{D}}$ the artificial set of unknowns and $\left(B_{\mathcal{Q}}\right)_{\mathcal{Q} \in \mathfrak{Q}_{\mathcal{D}}}$ the set of matrices in $\mathcal{M}_{2, n_{\mathcal{D}}}(\mathbb{R})$ defined as follows:

- $\forall \mathcal{D} \in \mathfrak{D}_{\text {int }}$, we take $\delta^{\mathcal{D}}={ }^{t}\left(\delta_{\mathcal{K}}, \delta_{\mathcal{L}}, \delta_{\mathcal{K}^{*}}, \delta_{\mathcal{L}^{*}}\right) \in \mathcal{M}_{n_{\mathcal{D}}, 2}(\mathbb{R})$ and four matrices $B_{\mathcal{Q}}$ :

$$
\begin{aligned}
& B_{\mathcal{Q}_{\mathcal{K}, \mathcal{K}^{*}}}=\left(\frac{m_{\sigma_{\mathcal{K}}} \overrightarrow{\mathbf{n}}_{\sigma_{\mathcal{K}} \mathcal{K}^{*}}}{m_{\mathcal{Q}_{\mathcal{K}, \mathcal{K}^{*}}}}, 0, \frac{m_{\sigma_{\mathcal{K}^{*}}} \overrightarrow{\mathbf{n}}_{\sigma \mathcal{K}}}{m_{\mathcal{Q}_{\mathcal{K}, \mathcal{K}^{*}}}}, 0\right), B_{\mathcal{Q}_{\mathcal{K}, \mathcal{L}^{*}}}=\left(-\frac{m_{\sigma_{\mathcal{K}}} \overrightarrow{\mathbf{n}}_{\sigma_{\mathcal{K}^{\mathcal{K}}}}}{m_{\mathcal{Q}_{\mathcal{K}, \mathcal{L}^{*}}}}, 0,0, \frac{m_{\sigma_{\mathcal{L}^{*}}} \overrightarrow{\mathbf{n}}_{\sigma \mathcal{K}}}{m_{\mathcal{Q}_{\mathcal{K}, \mathcal{L}^{*}}}}\right), \\
& B_{\mathcal{Q}_{\mathcal{L}, \mathcal{L}^{*}}}=\left(0,-\frac{m_{\sigma_{\mathcal{L}}} \overrightarrow{\mathbf{n}}_{\sigma_{\mathcal{L}} \mathcal{K}^{*}}}{m_{\mathcal{Q}_{\mathcal{L}, \mathcal{L}^{*}}}}, 0,-\frac{m_{\sigma_{\mathcal{L}^{*}}} \overrightarrow{\mathbf{n}}_{\sigma \mathcal{K}}}{m_{\mathcal{Q}_{\mathcal{L}, \mathcal{L}^{*}}}}\right), B_{\mathcal{Q}_{\mathcal{L}, \mathcal{K}^{*}}}=\left(0, \frac{m_{\sigma_{\mathcal{L}}} \overrightarrow{\mathbf{n}}_{\sigma_{\mathcal{L}} \mathcal{K}^{*}}}{m_{\mathcal{Q}_{\mathcal{L}, \mathcal{K}^{*}}}},-\frac{m_{\sigma_{\mathcal{K}^{*}}} \overrightarrow{\mathbf{n}}_{\sigma \mathcal{K}}}{m_{\mathcal{Q}_{\mathcal{L}, \mathcal{K}^{*}}}}, 0\right) .
\end{aligned}
$$

- $\forall \mathcal{D} \in \mathfrak{D}_{\text {ext }}$, there is only two non-degenerate quarter diamonds in $\mathfrak{Q}_{\mathcal{D}}$, we take $\delta^{\mathcal{D}}=\left(\delta_{\mathcal{K}}\right) \in \mathcal{M}_{n_{\mathcal{D}}, 2}(\mathbb{R})$ and the two corresponding matrices $B_{\mathcal{Q}}$ are given by:

$$
B_{\mathcal{Q}_{\mathcal{K}, \mathcal{K}^{*}}}=\left(\frac{m_{\sigma_{\mathcal{K}}} \overrightarrow{\mathbf{n}}_{\sigma_{\mathcal{K}^{\mathcal{K}}}}}{m_{\mathcal{Q}_{\mathcal{K}, \mathcal{K}^{*}}}}\right), \quad B_{\mathcal{Q}_{\mathcal{K}, \mathcal{L}^{*}}}=\left(-\frac{m_{\sigma_{\mathcal{K}}} \overrightarrow{\mathbf{n}}_{\sigma_{\mathcal{K}} \mathcal{K}^{*}}}{m_{\mathcal{Q}_{\mathcal{K}, \mathcal{L}^{*}}}}\right) .
$$

Thanks to the modified discrete gradient, we can define a modified symmetric operator a modified discrete strain rate tensor as follows.

DEFINITION 2.6 (Discrete strain rate tensor on quarter diamonds). A discrete strain rate tensor of a vector field of $\left(\mathbb{R}^{2}\right)^{\mathcal{T}}, \mathrm{D}_{\mathfrak{Q}}^{\mathcal{N}}:\left(\mathbb{R}^{2}\right)^{\mathcal{T}} \rightarrow\left(\mathrm{D}_{\mathcal{Q}}^{\mathcal{N}} \mathbf{u}^{\mathcal{T}}\right)_{\mathcal{Q} \in \mathfrak{Q}} \in\left(\mathcal{M}_{2}(\mathbb{R})\right)^{\mathfrak{Q}}$, is a set of tensor defined by: $\mathrm{D}_{\mathcal{Q}}^{\mathcal{N}} \mathbf{u}^{\mathcal{T}}=\frac{1}{2}\left(\nabla_{\mathcal{Q}}^{\mathcal{N}} \mathbf{u}^{\mathcal{T}}+{ }^{t}\left(\nabla_{\mathcal{Q}}^{\mathcal{N}} \mathbf{u}^{\mathcal{T}}\right)\right)$, for any $\mathcal{Q} \in \mathfrak{Q}$. It can be also written as $\mathrm{D}_{\mathcal{Q}}^{\mathcal{N}} \mathbf{u}^{\mathcal{T}}=\mathrm{D}^{\mathcal{D}} \mathbf{u}^{\mathcal{T}}+\frac{1}{2}\left({ }^{t} \delta^{\mathcal{D} t} B_{\mathcal{Q}}+B_{\mathcal{Q}} \delta^{\mathcal{D}}\right)$, for any $\mathcal{D} \in \mathfrak{D}$ and for any $\mathcal{Q} \in \mathfrak{Q}_{\mathcal{D}}$.

Furthermore, we easily see from the formulas above that $\sum_{\mathcal{Q} \in \mathfrak{Q}_{\mathcal{D}}} m_{\mathcal{Q}} B_{\mathcal{Q}}=0$ for any diamond cell $\mathcal{D}$. Hence the following straightforward result holds

LEMMA 2.1. For all $\mathcal{D} \in \mathfrak{D}$, for any $\xi \in \mathcal{M}_{2}(\mathbb{R})$, for any $\delta \in \mathcal{M}_{2, n_{\mathcal{D}}}(\mathbb{R})$, we have

$$
\xi=\frac{1}{m_{\mathcal{D}}} \sum_{\mathcal{Q} \in \mathfrak{Q}_{\mathcal{D}}} m_{\mathcal{Q}}\left(\xi+\frac{1}{2}\left(B_{\mathcal{Q}} \delta+{ }^{t} \delta^{t} B_{\mathcal{Q}}\right)\right) .
$$


Even if we do not yet determine the value of $\delta^{\mathcal{D}}$, this Lemma implies that the operators $\mathrm{D}^{\mathcal{D}}$ and $\mathrm{D}_{\mathcal{Q}}^{\mathcal{N}}, \nabla^{\mathcal{D}}$ and $\nabla_{\mathcal{Q}}^{\mathcal{N}}$ satisfy the following identities:

$$
\mathrm{D}^{\mathcal{D}} \mathbf{u}^{\mathcal{T}}=\frac{1}{m_{\mathcal{D}}} \sum_{\mathcal{Q} \in \mathfrak{Q}_{\mathcal{D}}} m_{\mathcal{Q}} \mathrm{D}_{\mathcal{Q}}^{\mathcal{N}} \mathbf{u}^{\mathcal{T}}, \quad \nabla^{\mathcal{D}} \mathbf{u}^{\mathcal{T}}=\frac{1}{m_{\mathcal{D}}} \sum_{\mathcal{Q} \in \mathfrak{Q}_{\mathcal{D}}} m_{\mathcal{Q}} \nabla_{\mathcal{Q}}^{\mathcal{N}} \mathbf{u}^{\mathcal{T}}, \quad \forall \mathcal{D} \in \mathfrak{D} .
$$

Thanks to the modified discrete strain rate tensor, we can define a modified viscous stress tensor and a complete discrete stress tensor as follows.

DEFinition 2.7 (Discrete viscous stress tensor on quarter diamonds). A modified discrete viscous stress tensor of a vector field of $\left(\mathbb{R}^{2}\right)^{\mathcal{T}}, \mathrm{D}_{\mathfrak{D}}^{\eta, \mathcal{N}}: \mathbf{u}^{\mathcal{T}} \in\left(\mathbb{R}^{2}\right)^{\mathcal{T}} \rightarrow\left(\mathrm{D}_{\mathcal{D}}^{\eta, \mathcal{N}} \mathbf{u}^{\mathcal{T}}\right)_{\mathcal{D} \in \mathfrak{D}} \in$ $\left(\mathcal{M}_{2}(\mathbb{R})\right)^{\mathfrak{D}}$, is defined by $\mathrm{D}_{\mathcal{D}}^{\eta, \mathcal{N}} \mathbf{u}^{\mathcal{T}}=\frac{1}{m_{\mathcal{D}}} \sum_{\mathcal{Q} \in \mathfrak{Q}_{\mathcal{D}}} m_{\mathcal{Q}} \eta_{\mathcal{Q}} \mathrm{D}_{\mathcal{Q}}^{\mathcal{N}} \mathbf{u}^{\mathcal{T}}$, for any $\mathcal{D} \in \mathfrak{D}$.

DEFINITION 2.8. We define a discrete stress tensor $\varphi_{\mathcal{Q}}$, for all $\mathcal{Q} \in \mathfrak{Q}_{\mathcal{D}}$ and for all $\mathcal{D} \in \mathfrak{D}$, by the formula: $\varphi_{\mathcal{Q}}\left(\mathrm{D}^{\mathcal{D}} \mathbf{u}^{\mathcal{T}}, \delta^{\mathcal{D}}, p^{\mathfrak{Q}_{\mathcal{D}}}\right)=2 \eta_{\mathcal{Q}} \mathrm{D}^{\mathcal{D}} \mathbf{u}^{\mathcal{T}}+\eta_{\mathcal{Q}}\left(B_{\mathcal{Q}} \delta^{\mathcal{D}}+{ }^{t} \delta^{\mathcal{D} t} B_{\mathcal{Q}}\right)-p^{\mathcal{Q}} \mathrm{Id}$.

2.4.3. Determination of the additional unknowns. On each diamond cell $\mathcal{D}$, we have $3 n_{\mathcal{D}}$ additional unknowns $\left(\delta^{\mathcal{D}}, p^{\mathfrak{Q}_{\mathcal{D}}}\right)$ that can be eliminated by imposing the conservativity of the numerical fluxes on all the diagonals of $\mathcal{D}$. The discrete counterpart of the conservativity condition (2.6) reads for any $\mathcal{D} \in \mathfrak{D}_{\text {int }}, \mathcal{D}=\mathcal{Q}_{\mathcal{K}, \mathcal{K}^{*}} \cup \mathcal{Q}_{\mathcal{K}, \mathcal{L}^{*}} \cup \mathcal{Q}_{\mathcal{L}, \mathcal{K}^{*}} \cup \mathcal{Q}_{\mathcal{L}, \mathcal{L}^{*}}$ :

$$
\left\{\begin{array}{l}
\varphi_{\mathcal{Q}_{\mathcal{K}, \mathcal{K}^{*}}}\left(\mathrm{D}^{\mathcal{D}} \mathbf{u}^{\mathcal{T}}, \delta^{\mathcal{D}}, p_{\mathcal{Q}_{\mathcal{K}, \mathcal{K}^{*}}}\right) \overrightarrow{\mathbf{n}}_{\sigma \mathcal{K}}=\varphi_{\mathcal{Q}_{\mathcal{L}, \mathcal{K}^{*}}}\left(\mathrm{D}^{\mathcal{D}} \mathbf{u}^{\mathcal{T}}, \delta^{\mathcal{D}}, p_{\mathcal{Q}_{\mathcal{L}, \mathcal{K}^{*}}}\right) \overrightarrow{\mathbf{n}}_{\sigma \mathcal{K}}, \\
\varphi_{\mathcal{Q}_{\mathcal{K}, \mathcal{L}^{*}}}\left(\mathrm{D}^{\mathcal{D}} \mathbf{u}^{\mathcal{T}}, \delta^{\mathcal{D}}, p_{\mathcal{Q}_{\mathcal{K}, \mathcal{L}^{*}}}\right) \overrightarrow{\mathbf{n}}_{\sigma \mathcal{K}}=\varphi_{\mathcal{Q}_{\mathcal{L}, \mathcal{L}^{*}}}\left(\mathrm{D}^{\mathcal{D}} \mathbf{u}^{\mathcal{T}}, \delta^{\mathcal{D}}, p_{\mathcal{Q}_{\mathcal{L}, \mathcal{L}^{*}}}\right) \overrightarrow{\mathbf{n}}_{\sigma \mathcal{K}}, \\
\varphi_{\mathcal{Q}_{\mathcal{K}, \mathcal{K}^{*}}}\left(\mathrm{D}^{\mathcal{D}} \mathbf{u}^{\mathcal{T}}, \delta^{\mathcal{D}}, p_{\mathcal{Q}_{\mathcal{K}, \mathcal{K}^{*}}}\right) \overrightarrow{\mathbf{n}}_{\sigma_{\mathcal{K}} \mathcal{K}^{*}}=\varphi_{\mathcal{Q}_{\mathcal{K}, \mathcal{L}^{*}}}\left(\mathrm{D}^{\mathcal{D}} \mathbf{u}^{\mathcal{T}}, \delta^{\mathcal{D}}, p_{\mathcal{Q}_{\mathcal{K}, \mathcal{L}^{*}}}\right) \overrightarrow{\mathbf{n}}_{\sigma_{\mathcal{K}} \mathcal{K}^{*}}, \\
\varphi_{\mathcal{Q}_{\mathcal{L}, \mathcal{K}^{*}}}\left(\mathrm{D}^{\mathcal{D}} \mathbf{u}^{\mathcal{T}}, \delta^{\mathcal{D}}, p_{\mathcal{Q}_{\mathcal{L}, \mathcal{K}^{*}}}\right) \overrightarrow{\mathbf{n}}_{\sigma_{\mathcal{L}} \mathcal{K}^{*}}=\varphi_{\mathcal{Q}_{\mathcal{L}, \mathcal{L}^{*}}}\left(\mathrm{D}^{\mathcal{D}} \mathbf{u}^{\mathcal{T}}, \delta^{\mathcal{D}}, p_{\mathcal{Q}_{\mathcal{L}, \mathcal{L}^{*}}}\right) \overrightarrow{\mathbf{n}}_{\sigma_{\mathcal{L}} \mathcal{K}^{*}},
\end{array}\right.
$$

and for any $\mathcal{D} \in \mathfrak{D}_{\text {ext }}, \quad \mathcal{D}=\mathcal{Q}_{\mathcal{K}, \mathcal{K}^{*}} \cup \mathcal{Q}_{\mathcal{K}, \mathcal{L}^{*}}$ :

$$
\varphi_{\mathcal{Q}_{\mathcal{K}, \mathcal{K}^{*}}}\left(\mathrm{D}^{\mathcal{D}} \mathbf{u}^{\mathcal{T}}, \delta^{\mathcal{D}}, p_{\mathcal{Q}_{\mathcal{K}, \mathcal{K}^{*}}}\right) \overrightarrow{\mathbf{n}}_{\sigma_{\mathcal{K}} \mathcal{K}^{*}}=\varphi_{\mathcal{Q}_{\mathcal{K}, \mathcal{L}^{*}}}\left(\mathrm{D}^{\mathcal{D}} \mathbf{u}^{\mathcal{T}}, \delta^{\mathcal{D}}, p_{\mathcal{Q}_{\mathcal{K}, \mathcal{L}^{*}}}\right) \overrightarrow{\mathbf{n}}_{\sigma_{\mathcal{K}} \mathcal{K}^{*}}
$$

It gives $2 n_{\mathcal{D}}$ equations, thus the linear system is underdetermined. We will add other conditions, remembering that we consider incompressible flows so the velocity satisfies divu $=0$. In the DDFV scheme, we add a stabilization term in order to prove its well-posedness. Thus, at the discrete level we do not have $\operatorname{div}^{\mathfrak{D}} \mathbf{u}^{\mathcal{T}}$ equal to zero. Nevertheless, we want the following equality to be verified $\operatorname{Tr}\left(\nabla_{\mathcal{Q}}^{\mathcal{N}} \mathbf{u}^{\mathcal{T}}\right)=\operatorname{div}^{\mathcal{D}} \mathbf{u}^{\mathcal{T}}$, for any $\mathcal{Q} \in \mathfrak{Q}_{\mathcal{D}}$ and for any $\mathcal{D} \in \mathfrak{D}$. As a result, we impose that

$$
\operatorname{Tr}\left({ }^{t} \delta^{\mathcal{D} t} B_{\mathcal{Q}}\right)=0, \quad \forall \mathcal{Q} \in \mathfrak{Q}_{\mathcal{D}}
$$

Since $\sum_{\mathcal{Q} \in \mathfrak{Q}_{\mathcal{D}}} m_{\mathcal{Q}} B_{\mathcal{Q}}=0$, we have that these equations are linked and so we add that

$$
\sum_{\mathcal{Q} \in \mathfrak{Q}_{\mathcal{D}}} m_{\mathcal{Q}} p^{\mathcal{Q}}=m_{\mathcal{D}} p^{\mathcal{D}}
$$

Note that the existence of $\left(\delta^{\mathcal{D}}, p^{\mathfrak{Q}_{\mathcal{D}}}\right)$ is not a straightforward adaptation of the proof in [5], since we use the discrete strain rate tensor and not the full discrete gradient. We need to first study the overdetermined linear system: for $F_{\mathcal{Q}} \in \mathcal{M}_{2}(\mathbb{R})$ given, can we find $\delta^{\mathcal{D}} \in$ $\mathcal{M}_{n_{\mathcal{D}}, 2}(\mathbb{R})$ such that

$$
{ }^{t} \delta^{\mathcal{D} t} B_{\mathcal{Q}}+B_{\mathcal{Q}} \delta^{\mathcal{D}}=F_{\mathcal{Q}}, \quad \forall \mathcal{Q} \in \mathfrak{Q}_{\mathcal{D}}
$$

PROPOSITION 2.1. If $F_{\mathcal{Q}}=0$, for all $\mathcal{Q} \in \mathfrak{Q}_{\mathcal{D}}$, the solutions of (2.12) are generated by $\delta_{0} \in \mathcal{M}_{n_{\mathcal{D}}, 2}(\mathbb{R}):$ 
- $\delta_{0}=0$ when $\alpha_{\mathcal{K}} \neq \alpha_{\mathcal{L}}$, (angles defined on Fig. 2.2(a)).

- $\delta_{0}=\left(-\frac{\overrightarrow{\mathbf{n}}_{\sigma \mathcal{K}}}{m_{\sigma_{\mathcal{K}}}}, \frac{\overrightarrow{\mathbf{n}}_{\sigma \mathcal{K}}}{m_{\sigma_{\mathcal{L}}}}, \frac{\overrightarrow{\mathbf{n}}_{\sigma^{*} \mathcal{K}^{*}}}{m_{\sigma_{\mathcal{K}^{*}}}},-\frac{\overrightarrow{\mathbf{n}}_{\sigma^{*} \mathcal{K}^{*}}}{m_{\sigma_{\mathcal{L}^{*}}}}\right)$ when $\alpha_{\mathcal{K}}=\alpha_{\mathcal{L}}$.

PROPOSITION 2.2. Under the following assumptions

$$
\left\{\begin{array}{l}
F_{\mathcal{Q}} \text { is symmetric for all } \mathcal{Q} \in \mathfrak{Q}_{\mathcal{D}} \text { and } \sum_{\mathcal{Q} \in \mathfrak{Q}_{\mathcal{D}}} m_{\mathcal{Q}} F_{\mathcal{Q}}=0, \\
m_{\mathcal{Q}_{\mathcal{K}, \mathcal{K}^{*}}}{ }^{t} \overrightarrow{\boldsymbol{\tau}}_{\mathcal{K}, \mathcal{L}} F_{\mathcal{Q}_{\mathcal{K}, \mathcal{K}^{*}}} \vec{\tau}_{\mathcal{K}, \mathcal{L}}+m_{\mathcal{Q}_{\mathcal{K}, \mathcal{L}^{*}}}{ }^{t} \overrightarrow{\boldsymbol{\tau}}_{\mathcal{K}, \mathcal{L}} F_{\mathcal{Q}_{\mathcal{K}, \mathcal{L}^{*}}} \vec{\tau}_{\mathcal{K}, \mathcal{L}}=0,
\end{array}\right.
$$

- When $\alpha_{\mathcal{K}}=\alpha_{\mathcal{L}}$, the system (2.12) admits a solution (non unique) if we have the additional assumption:

$$
m_{\mathcal{Q}_{\mathcal{K}, \mathcal{K}^{*}}}{ }^{t} \overrightarrow{\boldsymbol{\tau}}_{\mathcal{K}^{*}, \mathcal{L}^{*}} F_{\mathcal{Q}_{\mathcal{K}, \mathcal{K}^{*}}} \vec{\tau}_{\mathcal{K}^{*}, \mathcal{L}^{*}}+m_{\mathcal{Q}_{\mathcal{L}, \mathcal{K}^{*}}}{ }^{t} \overrightarrow{\boldsymbol{\tau}}_{\mathcal{K}^{*}, \mathcal{L}^{*}} F_{\mathcal{Q}_{\mathcal{L}, \mathcal{K}^{*}}} \vec{\tau}_{\mathcal{K}^{*}, \mathcal{L}^{*}}=0 .
$$

The solution is unique if we impose the orthogonality condition $\left(\delta^{\mathcal{D}}: \delta_{0}\right)=0$.

- When $\alpha_{\mathcal{K}} \neq \alpha_{\mathcal{L}}$, the system (2.12) admits an unique solution. Notice that we obviously have $\left(\delta^{\mathcal{D}}: \delta_{0}\right)=0$, since, in that case, we let $\delta_{0}=0$.

Proposition 2.3. For any $\mathcal{D} \in \mathfrak{D}$, the conditions (2.8) or (2.9), with (2.10)-(2.11) and $\left(\delta^{\mathcal{D}}: \delta_{0}\right)=0$ are equivalent to

$$
\begin{aligned}
& \sum_{\mathcal{Q} \in \mathfrak{Q}_{\mathcal{D}}} m_{\mathcal{Q}} \varphi_{\mathcal{Q}}\left(\mathrm{D}^{\mathcal{D}} \mathbf{u}^{\mathcal{T}}, \delta^{\mathcal{D}}, p^{\mathcal{Q}}\right) B_{\mathcal{Q}}=0, \\
& \operatorname{Tr}\left({ }^{t} \delta^{\mathcal{D} t} B_{\mathcal{Q}}\right)=0, \forall \mathcal{Q} \in \mathfrak{Q}_{\mathcal{D}}, \quad \sum_{\mathcal{Q} \in \mathfrak{Q}_{\mathcal{D}}} m_{\mathcal{Q}} p^{\mathcal{Q}}=m_{\mathcal{D}} p^{\mathcal{D}}, \\
& \left(\delta^{\mathcal{D}}: \delta_{0}\right)=0,
\end{aligned}
$$

where $\delta_{0}$ is defined in Proposition 2.1.

We are now able to prove the existence and uniqueness of a suitable choice for $\left(\delta^{\mathcal{D}}, p^{\mathfrak{Q}_{\mathcal{D}}}\right) \in$ $\mathcal{M}_{n_{\mathcal{D}}, 2}(\mathbb{R}) \times \mathbb{R}^{n_{\mathcal{D}}}$.

THEOREM 2.1. For any $\mathcal{D} \in \mathfrak{D}$ and for any $\left(\mathrm{D}^{\mathcal{D}} \mathbf{u}^{\mathcal{T}}, p^{\mathcal{D}}\right) \in \mathcal{M}_{2}(\mathbb{R}) \times \mathbb{R}$, there exists $a$ unique pair $\left(\delta^{\mathcal{D}}, p^{\mathfrak{Q}_{\mathcal{D}}}\right) \in \mathcal{M}_{n_{\mathcal{D}}, 2}(\mathbb{R}) \times \mathbb{R}^{n_{\mathcal{D}}}$ satisfying $(2.15)$.

Proof. We only give the proof for $\mathcal{D} \in \mathfrak{D}_{\text {int }}$ (so that $n_{\mathcal{D}}=4$ ), since the case of boundary diamond cells can be treated in the same way. We can write the system (2.15) like a linear rectangle system $A X=b$ with $A \in \mathcal{M}_{14,12}(\mathbb{R})$ and $b \in \mathbb{R}^{14}$, written as follows:

$$
b=\left(\begin{array}{c}
2\left(\eta_{\mathcal{Q}_{\mathcal{K}, \mathcal{K}^{*}}}-\eta_{\mathcal{Q}_{\mathcal{L}, \mathcal{K}^{*}}}\right) \mathrm{D}^{\mathcal{D}} \mathbf{u}^{\mathcal{T}} \overrightarrow{\mathbf{n}}_{\sigma \mathcal{K}} \\
2\left(\eta_{\mathcal{Q}_{\mathcal{K}, \mathcal{L}^{*}}}-\eta_{\mathcal{Q}_{\mathcal{L}, \mathcal{L}^{*}}}\right) \mathrm{D}^{\mathcal{D}} \mathbf{u}^{\mathcal{T}} \overrightarrow{\mathbf{n}}_{\sigma \mathcal{K}} \\
2\left(\eta_{\mathcal{Q}_{\mathcal{K}, \mathcal{K}^{*}}}-\eta_{\mathcal{Q}_{\mathcal{K}, \mathcal{L}^{*}}}\right) \mathrm{D}^{\mathcal{D}} \mathbf{u}^{\mathcal{T}} \overrightarrow{\mathbf{n}}_{\sigma \mathcal{K}} \mathcal{K}^{*} \\
2\left(\eta_{\mathcal{Q}_{\mathcal{L}, \mathcal{K}^{*}}}-\eta_{\mathcal{Q}_{\mathcal{L}, \mathcal{L}^{*}}}\right) \mathrm{D}^{\mathcal{D}} \mathbf{u}^{\mathcal{T}} \overrightarrow{\mathbf{n}}_{\sigma_{\mathcal{K}} \mathcal{K}^{*}} \\
0 \\
0 \\
0 \\
0 \\
m_{\mathcal{D}} p^{\mathcal{D}} \\
0
\end{array}\right) \in \mathbb{R}^{14} .
$$

We are interested in the kernel of $A$. We assume that $\mathrm{D}^{\mathcal{D}} \mathbf{u}^{\mathcal{T}}$ and $p^{\mathcal{D}}$ are zero, thus the second member $b$ is zero. Right-multiplying (2.15a) by $\delta^{\mathcal{D}}$ and taking the trace, it gives

$$
\sum_{\mathcal{Q} \in \mathfrak{Q}_{\mathcal{D}}} m_{\mathcal{Q}}\left(\varphi_{\mathcal{Q}}\left(\mathrm{D}^{\mathcal{D}} \mathbf{u}^{\mathcal{T}}, \delta^{\mathcal{D}}, p^{\mathcal{Q}}\right): B_{\mathcal{Q}} \delta^{\mathcal{D}}\right)=0
$$

Using Definition 2.8 of $\varphi_{\mathcal{Q}}$ and the fact it is a symmetric matrix, we have

$$
\sum_{\mathcal{Q} \in \mathfrak{Q}_{\mathcal{D}}} m_{\mathcal{Q}}\left(2 \eta_{\mathcal{Q}} \mathrm{D}^{\mathcal{D}} \mathbf{u}^{\mathcal{T}}+\eta_{\mathcal{Q}}\left(B_{\mathcal{Q}} \delta^{\mathcal{D}}+{ }^{t} \delta^{\mathcal{D} t} B_{\mathcal{Q}}\right)-p^{\mathcal{Q}} \operatorname{Id}: B_{\mathcal{Q}} \delta^{\mathcal{D}}+{ }^{t} \delta^{\mathcal{D} t} B_{\mathcal{Q}}\right)=0
$$


Furthermore we have $\left(\mathrm{Id}: B_{\mathcal{Q}} \delta^{\mathcal{D}}+{ }^{t} \delta^{\mathcal{D} t} B_{\mathcal{Q}}\right)=\operatorname{Tr}\left(B_{\mathcal{Q}} \delta^{\mathcal{D}}\right)=0$, it implies that

$$
\sum_{\mathcal{Q} \in \mathfrak{Q}_{\mathcal{D}}} m_{\mathcal{Q}}\left(2 \eta_{\mathcal{Q}} \mathrm{D}^{\mathcal{D}} \mathbf{u}^{\mathcal{T}}+\eta_{\mathcal{Q}}\left(B_{\mathcal{Q}} \delta^{\mathcal{D}}+{ }^{t} \delta^{\mathcal{D} t} B_{\mathcal{Q}}\right): B_{\mathcal{Q}} \delta^{\mathcal{D}}\right)=0
$$

Thanks to $\mathrm{D}^{\mathcal{D}} \mathbf{u}^{\mathcal{T}}=0$, we get

$$
\sum_{\mathcal{Q} \in \mathfrak{Q}_{\mathcal{D}}} m_{\mathcal{Q}} \eta_{\mathcal{Q}}\left({ }^{t} \delta^{\mathcal{D} t} B_{\mathcal{Q}}+B_{\mathcal{Q}} \delta^{\mathcal{D}}: B_{\mathcal{Q}} \delta^{\mathcal{D}}\right)=0
$$

Remarking that the Frobenius scalar product of a symmetric and antisymmetric matrix is equal to zero, we deduce $\sum_{\mathcal{Q} \in \mathfrak{Q}_{\mathcal{D}}} m_{\mathcal{Q}} \eta_{\mathcal{Q}}\left\|^{t} \delta^{\mathcal{D} t} B_{\mathcal{Q}}+B_{\mathcal{Q}} \delta^{\mathcal{D}}\right\|_{\mathcal{F}}^{2}=0$. Therefore, it implies

$$
{ }^{t} \delta^{\mathcal{D} t} B_{\mathcal{Q}}+B_{\mathcal{Q}} \delta^{\mathcal{D}}=0, \quad \forall \mathcal{Q} \in \mathfrak{Q}_{\mathcal{D}}
$$

Using the fact that $\left(\delta^{\mathcal{D}}: \delta_{0}\right)=0$, Proposition 2.1 implies that $\delta^{\mathcal{D}}=0$. Furthermore the condition (2.8) reduces to

$$
\begin{aligned}
& \left(p_{\mathcal{Q}_{\mathcal{K}, \mathcal{K}^{*}}}-p_{\mathcal{Q}_{\mathcal{L}, \mathcal{K}^{*}}}\right) \overrightarrow{\mathbf{n}}_{\sigma \mathcal{K}}=0, \quad\left(p_{\mathcal{Q}_{\mathcal{K}, \mathcal{L}^{*}}}-p_{\mathcal{Q}_{\mathcal{L}, \mathcal{L}^{*}}}\right) \overrightarrow{\mathbf{n}}_{\sigma \mathcal{K}}=0, \\
& \left(p_{\mathcal{Q}_{\mathcal{K}, \mathcal{K}^{*}}}-p_{\mathcal{Q}_{\mathcal{K}, \mathcal{L}^{*}}}\right) \overrightarrow{\mathbf{n}}_{\sigma_{\mathcal{K}} \mathcal{K}^{*}}=0, \quad\left(p_{\mathcal{Q}_{\mathcal{L}, \mathcal{K}^{*}}}-p_{\mathcal{Q}_{\mathcal{L}, \mathcal{L}^{*}}}\right) \overrightarrow{\mathbf{n}}_{\sigma_{\mathcal{L}} \mathcal{K}^{*}}=0 .
\end{aligned}
$$

We obtain that $p_{\mathcal{Q}_{\mathcal{K}, \mathcal{K}^{*}}}=p_{\mathcal{Q}_{\mathcal{L}, \mathcal{K}^{*}}}=p_{\mathcal{Q}_{\mathcal{K}, \mathcal{L}^{*}}}=p_{\mathcal{Q}_{\mathcal{L}, \mathcal{L}^{*}}}$ and thanks to $(2.11)$, we get $p^{\mathfrak{Q}_{\mathcal{D}}}=0$. It remains to study the kernel of the adjoint of the matrix $A$. We need to differentiate two cases.

- Case $\alpha_{\mathcal{K}} \neq \alpha_{\mathcal{L}}$. We observe that the kernel of the adjoint $\operatorname{Ker}^{t} A=\operatorname{Span} X_{1}$ where:

$$
X_{1}={ }^{t}\left(0, \cdots, 0, m_{\mathcal{Q}_{\mathcal{K}, \mathcal{K}^{*}}}, m_{\mathcal{Q}_{\mathcal{K}, \mathcal{L}^{*}}}, m_{\mathcal{Q}_{\mathcal{L}, \mathcal{K}^{*}}}, m_{\mathcal{Q}_{\mathcal{L}, \mathcal{L}^{*}}}, 0,0\right) \in \mathbb{R}^{14} .
$$

We immediately get that $\left(X_{1}, b\right)=0$, where $b$ is given by (2.16). So that we have $b \in$ $\left(\operatorname{Ker}^{t} A\right)^{\perp}=\operatorname{Im} A$ and we deduce the existence of $\left(\delta^{\mathcal{D}}, p^{\mathfrak{Q}_{\mathcal{D}}}\right)$.

- Case $\alpha_{\mathcal{K}}=\alpha_{\mathcal{L}}$. We determine the kernel of the adjoint $\operatorname{Ker}^{t} A=\operatorname{Span}\left(X_{1}, X_{2}\right)$ where $X_{1}$ is given above and

$$
X_{2}={ }^{t}\left(-{ }^{t} \overrightarrow{\mathbf{n}}_{\sigma_{\mathcal{K}} \mathcal{K}^{*}},{ }^{t} \overrightarrow{\mathbf{n}}_{\sigma_{\mathcal{K}} \mathcal{K}^{*}},{ }^{t} \overrightarrow{\mathbf{n}}_{\sigma \mathcal{K}},-{ }^{t} \overrightarrow{\mathbf{n}}_{\sigma \mathcal{K}}, 0, \cdots, 0\right) \in \mathbb{R}^{14} .
$$

We have to prove once again that $b \in \operatorname{Im} A=\left(\operatorname{Ker}^{t} A\right)^{\perp}$. We still have $\left(X_{1}, b\right)=0$. We just have to prove that $\left(X_{2}, b\right)=0$, thus we compute

$$
\begin{aligned}
\left(X_{2}, b\right)= & -2\left(\eta_{\mathcal{Q}_{\mathcal{K}, \mathcal{K}^{*}}}-\eta_{\mathcal{Q}_{\mathcal{L}, \mathcal{K}^{*}}}\right)\left(\mathrm{D}^{\mathcal{D}} \mathbf{u}^{\mathcal{T}} \overrightarrow{\mathbf{n}}_{\sigma \mathcal{K}}, \overrightarrow{\mathbf{n}}_{\sigma_{\mathcal{K}} \mathcal{K}^{*}}\right)+2\left(\eta_{\mathcal{Q}_{\mathcal{K}, \mathcal{L}^{*}}}-\eta_{\mathcal{Q}_{\mathcal{L}, \mathcal{L}^{*}}}\right)\left(\mathrm{D}^{\mathcal{D}} \mathbf{u}^{\mathcal{T}} \overrightarrow{\mathbf{n}}_{\sigma \mathcal{K}}, \overrightarrow{\mathbf{n}}_{\sigma_{\mathcal{K}} \mathcal{K}^{*}}\right) \\
& +2\left(\eta_{\mathcal{Q}_{\mathcal{K}, \mathcal{K}^{*}}}-\eta_{\mathcal{Q}_{\mathcal{K}, \mathcal{L}^{*}}}\right)\left(\mathrm{D}^{\mathcal{D}} \mathbf{u}^{\mathcal{T}} \overrightarrow{\mathbf{n}}_{\sigma_{\mathcal{K}} \mathcal{K}^{*}}, \overrightarrow{\mathbf{n}}_{\sigma \mathcal{K}}\right)-2\left(\eta_{\mathcal{Q}_{\mathcal{L}, \mathcal{K}^{*}}}-\eta_{\mathcal{Q}_{\mathcal{L}, \mathcal{L}^{*}}}\right)\left(\mathrm{D}^{\mathcal{D}} \mathbf{u}^{\mathcal{T}} \overrightarrow{\mathbf{n}}_{\sigma_{\mathcal{K}} \mathcal{K}^{*}}, \overrightarrow{\mathbf{n}}_{\sigma \mathcal{K}}\right) .
\end{aligned}
$$

Using the fact that $\mathrm{D}^{\mathcal{D}} \mathbf{u}^{\mathcal{T}}$ is symmetric, ie $\left(\mathrm{D}^{\mathcal{D}} \mathbf{u}^{\mathcal{T}} \overrightarrow{\mathbf{n}}_{\sigma \mathcal{K}}, \overrightarrow{\mathbf{n}}_{\sigma_{\mathcal{K}} \mathcal{K}^{*}}\right)=\left(\mathrm{D}^{\mathcal{D}} \mathbf{u}^{\mathcal{T}} \overrightarrow{\mathbf{n}}_{\sigma_{\mathcal{K}} \mathcal{K}^{*}}, \overrightarrow{\mathbf{n}}_{\sigma \mathcal{K}}\right)$, we deduce $\left(X_{2}, b\right)=0$. Therefore $b \in \operatorname{Im} A$, we deduce the existence of $\left(\delta^{\mathcal{D}}, p^{\mathfrak{Q}_{\mathcal{D}}}\right)$.

From now on, the artificial unknowns $\left(\delta^{\mathcal{D}}, p^{\mathfrak{Q}_{\mathcal{D}}}\right)$ are determined, they linearly depend on $\left(\mathrm{D}^{\mathcal{D}} \mathbf{u}^{\mathcal{T}}, p^{\mathcal{D}}\right)$. Thus the modified discrete gradient $\nabla_{\mathfrak{Q}}^{\mathcal{N}}$ and the modified discrete strain rate tensor $\mathrm{D}_{\mathfrak{Q}}^{\mathcal{N}}$ are completely determined for all $\mathcal{Q} \in \mathfrak{Q}_{\mathcal{D}}$ and $\mathcal{D} \in \mathfrak{D}$ :

$$
\begin{aligned}
& \nabla_{\mathcal{Q}}^{\mathcal{N}} \mathbf{u}^{\mathcal{T}}=\nabla^{\mathcal{D}} \mathbf{u}^{\mathcal{T}}+{ }^{t} \delta^{\mathcal{D}}\left(\mathrm{D}^{\mathcal{D}} \mathbf{u}^{\mathcal{T}}, p^{\mathcal{D}}\right)^{t} B_{\mathcal{Q}} \\
& \mathrm{D}_{\mathcal{Q}}^{\mathcal{N}} \mathbf{u}^{\mathcal{T}}=\mathrm{D}^{\mathcal{D}} \mathbf{u}^{\mathcal{T}}+B_{\mathcal{Q}} \delta^{\mathcal{D}}\left(\mathrm{D}^{\mathcal{D}} \mathbf{u}^{\mathcal{T}}, p^{\mathcal{D}}\right)+{ }^{t} \delta^{\mathcal{D}}\left(\mathrm{D}^{\mathcal{D}} \mathbf{u}^{\mathcal{T}}, p^{\mathcal{D}}\right){ }^{t} B_{\mathcal{Q}}, \\
& \operatorname{Tr}\left(\nabla_{\mathcal{Q}}^{\mathcal{N}} \mathbf{u}^{\mathcal{T}}\right)=\operatorname{div}^{\mathcal{D}}\left(\mathbf{u}^{\mathcal{T}}\right) .
\end{aligned}
$$




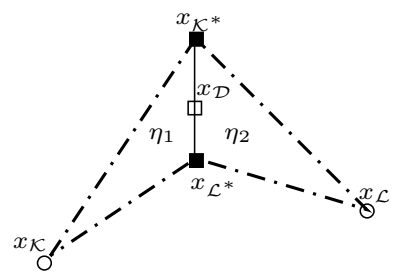

FIG. 2.5. The viscosity on $\mathcal{D}$ when $\mathcal{D} \cap \Gamma \neq \emptyset$.

2.4.4. Example of the artificial unknowns. Let us illustrate the value of the artificial unknowns $\left(\delta^{\mathcal{D}}, p^{\mathfrak{Q}_{\mathcal{D}}}\right)$ in the case where $\eta$ is constant per sub-domains, equal to $\eta_{1}$ on $\Omega_{1}$ and to $\eta_{2}$ on $\Omega_{2}$. For $\mathcal{D} \cap \Gamma \neq \emptyset$ (see Fig. 2.5), the solution $\left(\delta^{\mathcal{D}}, p^{\mathfrak{Q}_{\mathcal{D}}}\right)$ is equal to

$$
\begin{aligned}
& \delta_{\mathcal{K}}=\delta_{\mathcal{L}}=0, \quad \delta_{\mathcal{K}^{*}}=\delta_{\mathcal{L}^{*}}=-\frac{m_{\mathcal{Q}_{\mathcal{K}, \mathcal{K}^{*}}} m_{\mathcal{Q}_{\mathcal{L}, \mathcal{K}^{*}}}\left(\eta_{1}-\eta_{2}\right) \mathrm{D}^{\mathcal{D}} \mathbf{u}^{\boldsymbol{\tau}} \overrightarrow{\mathbf{n}}_{\sigma \mathcal{K}} \cdot \overrightarrow{\boldsymbol{\tau}}_{\mathcal{K}, \mathcal{L}}}{\eta_{2} m_{\mathcal{Q}_{\mathcal{K}, \mathcal{K}^{*}}}+\eta_{1} m_{\mathcal{Q}_{\mathcal{L}, \mathcal{K}^{*}}}} \vec{\tau}_{\mathcal{K}, \mathcal{L}} \\
& p_{\mathcal{Q}_{\mathcal{K}, \mathcal{K}^{*}}}=p^{\mathcal{D}}+2\left(\eta_{1}-\eta_{2}\right) \mathrm{D}^{\mathcal{D}} \mathbf{u}^{\mathcal{T}} \overrightarrow{\mathbf{n}}_{\sigma \mathcal{K}} \cdot \overrightarrow{\mathbf{n}}_{\sigma \mathcal{K}} \frac{m_{\mathcal{Q}_{\mathcal{L}, \mathcal{K}^{*}}}}{m_{\mathcal{Q}_{\mathcal{K}, \mathcal{K}^{*}}}+m_{\mathcal{Q}_{\mathcal{L}, \mathcal{K}^{*}}}}, \quad p_{\mathcal{Q}_{\mathcal{K}, \mathcal{L}^{*}}}=p_{\mathcal{Q}_{\mathcal{K}, \mathcal{K}^{*}}}, \\
& p_{\mathcal{Q}_{\mathcal{L}, \mathcal{K}^{*}}}=p^{\mathcal{D}}+2\left(\eta_{2}-\eta_{1}\right) \mathrm{D}^{\mathcal{D}} \mathbf{u}^{\mathcal{T}} \overrightarrow{\mathbf{n}}_{\sigma \mathcal{K}} \cdot \overrightarrow{\mathbf{n}}_{\sigma \mathcal{K}} \frac{m_{\mathcal{Q}_{\mathcal{K}, \mathcal{K}^{*}}}}{m_{\mathcal{Q}_{\mathcal{K}, \mathcal{K}^{*}}}+m_{\mathcal{Q}_{\mathcal{L}, \mathcal{K}^{*}}}}, \quad p_{\mathcal{Q}_{\mathcal{L}, \mathcal{L}^{*}}}=p_{\mathcal{Q}_{\mathcal{L}, \mathcal{K}^{*}}} .
\end{aligned}
$$

In that case, if we note the discrete strain rate tensor by $\mathrm{D}^{\mathcal{D}} \mathbf{u}^{\mathcal{T}}=\left(\begin{array}{ll}\alpha & \gamma \\ \gamma & \beta\end{array}\right)$, the modified discrete viscous stress tensor is equal to $\mathrm{D}_{\mathcal{D}}^{\eta, \mathcal{N}} \mathbf{u}^{\mathcal{T}}=\left(\begin{array}{cc}\frac{m_{\sigma_{\mathcal{K}} \eta_{1}+m_{\sigma} \mathcal{L} \eta_{2}}}{m_{\sigma_{\mathcal{K}}}+m_{\sigma} \mathcal{L}} \alpha & \frac{\left(m_{\sigma_{\mathcal{K}}}+m_{\sigma_{\mathcal{L}}}\right) \eta_{1} \eta_{2}}{m_{\sigma_{\mathcal{L}}} \eta_{1}+m_{\sigma_{\mathcal{K}}} \eta_{2}} \gamma \\ \frac{\left(m_{\left.\sigma_{\mathcal{K}}+m_{\sigma_{\mathcal{L}}}\right) \eta_{1} \eta_{2}}\right.}{m_{\sigma_{\mathcal{L}} \eta_{1}+m_{\sigma_{\mathcal{K}}} \eta_{2}}} \gamma & \frac{m_{\sigma_{\mathcal{K}}} \eta_{1}+m_{\sigma_{\mathcal{L}}} \eta_{2}}{m_{\sigma_{\mathcal{K}}}+m_{\sigma_{\mathcal{L}}}} \beta\end{array}\right)$ in the basis $\left(\overrightarrow{\mathbf{n}}_{\sigma \mathcal{K}}, \overrightarrow{\boldsymbol{\tau}}_{\mathcal{K}^{*}, \mathcal{L}^{*}}\right)$. We notice that $\mathrm{D}_{\mathcal{D}}^{\eta, \mathcal{N}} \mathbf{u}^{\mathcal{T}}$ is not proportional to $\mathrm{D}^{\mathcal{D}} \mathbf{u}^{\mathcal{T}}$. Diagonal terms are multiplied with the arithmetical mean of the viscosities where the off-diagonal terms are multiplied by the harmonic mean of the vicosities.

2.4.5. Implementation. We want to emphasize at this point that the implementation of the m-DDFV scheme is easy. To solve the linear system (3.1) which reads $\mathcal{A}\left(u^{\mathcal{T}}, p^{\mathfrak{D}}, \delta^{\mathfrak{D}}, p^{\mathfrak{Q}}\right)=$ $b$, we first calculate, for each diamond cell $\mathcal{D} \in \mathfrak{D}$, the pseudo-inverse the $12 \times 14$ matrix involved in (2.15). Thus the twelve artificial unknowns $\left(\delta^{\mathcal{D}}, p^{\mathfrak{Q}_{\mathcal{D}}}\right)$ can be expressed as a linear function of $\mathbf{u}^{\mathcal{T}}$ and $p^{\mathfrak{D}}$ let say $\left(\delta^{\mathcal{D}}, p^{\mathfrak{Q}_{\mathcal{D}}}\right)=f^{\mathcal{D}}\left(\mathbf{u}^{\mathcal{T}}, p^{\mathfrak{D}}\right)$, (see Section 2.4.4). This first procedure has little cost and can be easily vectorized/parallelized, since it is a local (per diamond) computation which has only to be done once at the beginning of the resolution. The second step consists then to rewrite the m-DDFV scheme (3.1) in term on the unknowns $\mathbf{u}^{\mathcal{T}}$ and $p^{\mathfrak{D}}$ thanks to the functions $f^{\mathcal{D}}$ and the modified fluxes which reads $\mathcal{A}_{\text {new }}\left(u^{\mathcal{T}}, p^{\mathfrak{D}}\right)=b$. The matrix $\mathcal{A}_{\text {new }}$ is then assembled diamond cell per diamond cell just like the scheme (1.4).

2.4.6. Properties of the artificial unknowns. First of all, we prove estimates between $B_{\mathcal{Q}} \delta^{\mathcal{D}}$ and $B_{\mathcal{Q}} \delta^{\mathcal{D}}+{ }^{t} \delta^{\mathcal{D} t} B_{\mathcal{Q}}$ that can be seen as a local Korn inequality on a diamond for the velocity artificial unknowns. Like in the proof of the existence of $\delta^{\mathcal{D}}$, the two cases $\alpha_{\mathcal{K}}=\alpha_{\mathcal{L}}$ and $\alpha_{\mathcal{K}} \neq \alpha_{\mathcal{L}}$ have to be investigated. The following Lemma is proved in Section 9.1.

LEMMA 2.2. For all $\mathcal{D} \in \mathfrak{D}$, for all $\delta^{\mathcal{D}} \in \mathcal{M}_{n_{\mathcal{D}}, 2}(\mathbb{R})$ such that $\left(\delta^{\mathcal{D}}: \delta_{0}\right)=0$, there exists $C_{1}>0$, depending only on $\operatorname{reg}(\mathcal{T})$ and $\sin \left(\epsilon_{0}\right)$, such that

$$
\sum_{\mathcal{Q} \in \mathfrak{Q}_{\mathcal{D}}} m_{\mathcal{Q}}\left\|B_{\mathcal{Q}} \delta^{\mathcal{D}}\right\|_{\mathcal{F}}^{2} \leq C_{1} \sum_{\mathcal{Q} \in \mathfrak{Q}_{\mathcal{D}}} m_{\mathcal{Q}}\left\|B_{\mathcal{Q}} \delta^{\mathcal{D}}+{ }^{t} \delta^{\mathcal{D} t} B_{\mathcal{Q}}\right\|_{\mathcal{F}}^{2},
$$

where $\delta_{0}$ is defined in Proposition 2.1. 
We bring out the form of artificial pressure unknowns $p^{\mathfrak{Q}_{\mathcal{D}}}$ in the following result proved in Section 9.2.

LEMmA 2.3. For any $\mathcal{D} \in \mathfrak{D}$, any $\left(\mathrm{D}^{\mathcal{D}} \mathbf{u}^{\mathcal{T}}, p^{\mathcal{D}}\right) \in \mathcal{M}_{2}(\mathbb{R}) \times \mathbb{R}$, there exists $C_{2}>0$, depending only on $\operatorname{reg}(\mathcal{T})$ and $\overline{\mathrm{C}}_{\eta}$, and a linear function $\alpha_{\mathfrak{s}, \mathcal{D}}$ such that the solution $\left(\delta^{\mathcal{D}}, p^{\mathfrak{Q}_{\mathcal{D}}}\right)$ of (2.15) with $\left(\mathrm{D}^{\mathcal{D}} \mathbf{u}^{\mathcal{T}}, p^{\mathcal{D}}\right)$, as the following form

$$
p^{\mathcal{Q}}=p^{\mathcal{D}}+\alpha_{\mathfrak{s}, \mathcal{D}}\left(\mathrm{D}^{\mathcal{D}} \mathbf{u}^{\mathcal{T}}\right), \quad \text { where } \mathfrak{s}=\partial \mathcal{D} \cap \partial \mathcal{Q},
$$

with $\left|\alpha_{\mathfrak{s}, \mathcal{D}}\left(q^{\mathcal{D}}\right)\right|^{2} \leq C_{2}\left\|q^{\mathcal{D}}\right\|_{\mathcal{F}}^{2}$, for any $q^{\mathcal{D}} \in \mathcal{M}_{2}(\mathbb{R})$.

2.5. Inner products and norms. We define the four following inner products

$$
\begin{aligned}
\llbracket \mathbf{v}^{\mathcal{T}}, \mathbf{u}^{\mathcal{T}} \rrbracket_{\mathcal{T}} & =\frac{1}{2}\left(\sum_{\mathcal{K} \in \mathfrak{M}} m_{\mathcal{K}} \mathbf{u}_{\mathcal{K}} \cdot \mathbf{v}_{\mathcal{K}}+\sum_{\mathcal{K}^{*} \in \mathfrak{M}^{*} \cup \partial \mathfrak{M}^{*}} m_{\mathcal{K}^{*}} \mathbf{u}_{\mathcal{K}^{*}} \cdot \mathbf{v}_{\mathcal{K}^{*}}\right), \quad \forall \mathbf{u}^{\mathcal{T}}, \mathbf{v}^{\mathcal{T}} \in\left(\mathbb{R}^{2}\right)^{\mathcal{T}}, \\
\left(p^{\mathfrak{D}}, q^{\mathfrak{D}}\right)_{\mathfrak{D}} & =\sum_{\mathcal{D} \in \mathfrak{D}} m_{\mathcal{D}} p^{\mathcal{D}} q^{\mathcal{D}}, \quad \forall p^{\mathfrak{D}}, q^{\mathfrak{D}} \in \mathbb{R}^{\mathfrak{D}}, \\
\left(\xi^{\mathfrak{D}}: \phi^{\mathfrak{D}}\right)_{\mathfrak{D}} & =\sum_{\mathcal{D} \in \mathfrak{D}} m_{\mathcal{D}}\left(\xi^{\mathcal{D}}: \phi^{\mathcal{D}}\right), \quad \forall \xi^{\mathfrak{D}}, \phi^{\mathfrak{D}} \in\left(\mathcal{M}_{2}(\mathbb{R})\right)^{\mathfrak{D}}, \\
\left(\xi^{\mathfrak{Q}}: \phi^{\mathfrak{Q}}\right)_{\mathfrak{Q}} & =\sum_{\mathcal{Q} \in \mathfrak{Q}} m_{\mathcal{Q}}\left(\xi^{\mathcal{Q}}: \phi^{\mathcal{Q}}\right), \quad \forall \xi^{\mathfrak{Q}}, \phi^{\mathfrak{Q}} \in\left(\mathcal{M}_{2}(\mathbb{R})\right)^{\mathfrak{Q}},
\end{aligned}
$$

and the corresponding norms:

$$
\begin{array}{ll}
\left\|\mathbf{u}^{\mathcal{T}}\right\|_{2}=\llbracket \mathbf{u}^{\mathcal{T}}, \mathbf{u}^{\mathcal{T}} \rrbracket_{\mathcal{T}}^{\frac{1}{2}}, & \forall \mathbf{u}^{\mathcal{T}} \in\left(\mathbb{R}^{2}\right)^{\mathcal{T}}, \\
\left\|p^{\mathfrak{D}}\right\|_{2}=\left(p^{\mathfrak{D}}, p^{\mathfrak{D}}\right)_{\mathfrak{D}}^{\frac{1}{2}}, \quad \forall p^{\mathfrak{D}} \in \mathbb{R}^{\mathfrak{D}}, \quad\left\|\xi^{\mathfrak{D}}\right\|_{2}=\left(\xi^{\mathfrak{D}}: \xi^{\mathfrak{D}}\right)_{\mathfrak{D}}^{\frac{1}{2}}, \quad \forall \xi^{\mathfrak{D}} \in\left(\mathcal{M}_{2}(\mathbb{R})\right)^{\mathfrak{D}}, \\
\left\|q^{\mathfrak{Q}}\right\|_{2}=\left(q^{\mathfrak{Q}}, q^{\mathfrak{Q}}\right)_{\mathfrak{Q}}^{\frac{1}{2}}, \quad \forall q^{\mathfrak{Q}} \in \mathbb{R}^{\mathfrak{Q}}, \quad\left\|\xi^{\mathfrak{Q}}\right\|_{2}=\left(\xi^{\mathfrak{Q}}: \xi^{\mathfrak{Q}}\right)_{\mathfrak{Q}}^{\frac{1}{2}}, \quad \forall \xi^{\mathfrak{Q}} \in\left(\mathcal{M}_{2}(\mathbb{R})\right)^{\mathfrak{Q}} .
\end{array}
$$

2.6. Preparation of the stabilization procedure. We define a second order discrete difference operator as follows.

DEFINITION 2.9. We define a second order discrete difference operator, denoted by $\Delta^{\mathfrak{D}}: p^{\mathfrak{D}} \in \mathbb{R}^{\mathfrak{D}} \mapsto \Delta^{\mathfrak{D}} p^{\mathfrak{D}} \in \mathbb{R}^{\mathfrak{D}}$, as follows:

$$
\Delta^{\mathcal{D}} p^{\mathfrak{D}}=\frac{1}{m_{\mathcal{D}}} \sum_{\mathfrak{s}=\mathcal{D} \mid \mathcal{D}^{\prime} \in \mathcal{E}_{\mathcal{D}}} \frac{h_{\mathcal{D}}^{2}+h_{\mathcal{D}^{\prime}}^{2}}{h_{\mathcal{D}}^{2}}\left(p^{\mathcal{D}^{\prime}}-p^{\mathcal{D}}\right), \quad \forall \mathcal{D} \in \mathfrak{D} .
$$

It is a non consistent approximation of the Laplace operator. Related to this operator, we define a mesh dependent semi-norm $|\cdot|_{h}$ over $\mathbb{R}^{\mathfrak{D}}$ by:

DEFINITION 2.10. We define a discrete semi-norm for any $p^{\mathfrak{D}} \in \mathbb{R}^{\mathfrak{D}}$ :

$$
\left|p^{\mathfrak{D}}\right|_{h}^{2}=\sum_{\mathfrak{s}=\mathcal{D} \mid \mathcal{D}^{\prime} \in \mathfrak{S}}\left(h_{\mathcal{D}}^{2}+h_{\mathcal{D}^{\prime}}^{2}\right)\left(p^{\mathcal{D}^{\prime}}-p^{\mathcal{D}}\right)^{2} .
$$

The semi-norm $|p|_{h}$ is the discrete counterpart of $\operatorname{size}(\mathcal{T})|\nabla p|_{2}$. We have that (see [21, Remark 3.6])

$$
-\left(h_{\mathfrak{D}}^{2} \Delta^{\mathfrak{D}} p^{\mathfrak{D}}, p^{\mathfrak{D}}\right)_{\mathfrak{D}}=\left|p^{\mathfrak{D}}\right|_{h}^{2}, \quad \forall p^{\mathfrak{D}} \in \mathbb{R}^{\mathfrak{D}} .
$$

Now we can define the new stabilization term, that considers the jumps of the pressure on quarter diamond cells.

DEFINITION 2.11. We define a second order discrete difference operator, denoted by $\underline{\Delta}^{\mathfrak{D}}: p^{\mathfrak{Q}} \in \mathbb{R}^{\mathfrak{Q}} \mapsto \underline{\Delta}^{\mathfrak{D}} p^{\mathfrak{Q}} \in \mathbb{R}^{\mathfrak{D}}$, as follows (see Fig. 2.6):

$$
\underline{\Delta}^{\mathcal{D}} p^{\mathfrak{Q}}=\frac{1}{m_{\mathcal{D}}} \sum_{\substack{\mathfrak{s}=\mathcal{Q}\left|\mathcal{Q}^{\prime} \\=\mathcal{D}\right| \mathcal{D}^{\prime} \in \mathcal{E}_{\mathcal{D}}}} \frac{h_{\mathcal{D}}^{2}+h_{\mathcal{D}^{\prime}}^{2}}{h_{\mathcal{D}}^{2}}\left(p^{\mathcal{Q}^{\prime}}-p^{\mathcal{Q}}\right), \quad \forall \mathcal{D} \in \mathfrak{D} .
$$




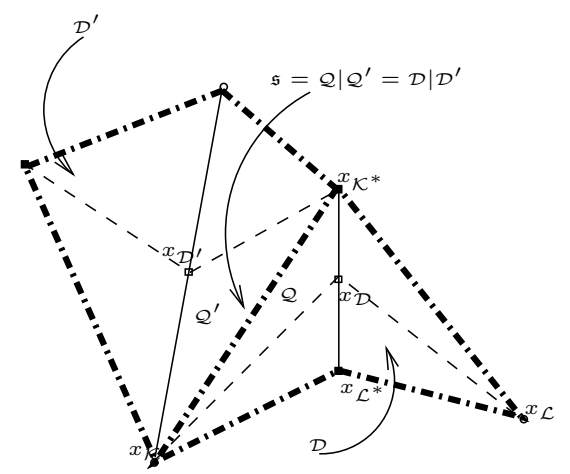

FIG. 2.6. A diamond cell $\mathcal{D}$ and its neighbouring diamond cell $\mathcal{D}^{\prime}$

It is also a non consistent approximation of the Laplace operator. Note that we do not need a consistent approximation of the Laplace operator. In fact, a consistent approximation based on a two-point flux formula would require the diamond mesh to verify an orthogonality constraint as, for instance, in the case of admissible meshes [13], which has no reason to hold here. An other operator uses the function $\alpha_{\mathfrak{s}, \mathcal{D}}$ introduced in Lemma 2.3 as follows.

DEFINITION 2.12. We define a second order discrete difference operator, denoted by $\Delta_{\alpha}^{\mathfrak{D}}: q^{\mathfrak{D}} \in\left(\mathcal{M}_{2}(\mathbb{R})\right)^{\mathfrak{D}} \mapsto \Delta_{\alpha}^{\mathfrak{D}} q^{\mathfrak{D}} \in \mathbb{R}^{\mathfrak{D}}$, as follows:

$$
\Delta_{\alpha}^{\mathcal{D}} q^{\mathfrak{D}}=\frac{1}{m_{\mathcal{D}}} \sum_{\mathfrak{s}=\mathcal{D} \mid \mathcal{D}^{\prime} \in \mathcal{E}_{\mathcal{D}}} \frac{h_{\mathcal{D}}^{2}+h_{\mathcal{D}^{\prime}}^{2}}{h_{\mathcal{D}}^{2}}\left(\alpha_{\mathfrak{s}, \mathcal{D}^{\prime}}\left(q^{\mathcal{D}^{\prime}}\right)-\alpha_{\mathfrak{s}, \mathcal{D}}\left(q^{\mathcal{D}}\right)\right), \forall \mathcal{D} \in \mathfrak{D},
$$

where $\alpha_{\mathfrak{s}, \mathcal{D}}$ is the function defined in Lemma 2.3.

DEFINITION 2.13. We define a discrete semi-norm for any $q^{\mathfrak{D}} \in\left(\mathcal{M}_{2}(\mathbb{R})\right)^{\mathfrak{D}}$ :

$$
\left|q^{\mathfrak{D}}\right|_{\alpha, h}^{2}:=\sum_{\mathfrak{s}=\mathcal{D} \mid \mathcal{D}^{\prime} \in \mathfrak{S}}\left(h_{\mathcal{D}}^{2}+h_{\mathcal{D}^{\prime}}^{2}\right)\left(\alpha_{\mathfrak{s}, \mathcal{D}^{\prime}}\left(q^{\mathcal{D}^{\prime}}\right)-\alpha_{\mathfrak{s}, \mathcal{D}}\left(q^{\mathcal{D}}\right)\right)^{2},
$$

where $\alpha_{\mathfrak{s}, \mathcal{D}}$ is the function defined in Lemma 2.3.

Thanks to the property $\left|\alpha_{\mathfrak{s}, \mathcal{D}}\left(q^{\mathcal{D}}\right)\right|^{2} \leq C_{2}\left\|q^{\mathcal{D}}\right\|_{\mathcal{F}}^{2}$ and relation (2.2), the Cauchy-Schwarz inequality implies that

$$
\left|q^{\mathfrak{D}}\right|_{\alpha, h}^{2} \leq C_{3}\left\|q^{\mathfrak{D}}\right\|_{2}^{2}, \quad \forall q^{\mathfrak{D}} \in\left(\mathcal{M}_{2}(\mathbb{R})\right)^{\mathfrak{D}},
$$

with $C_{3}=8 C_{2} \operatorname{reg}(\mathcal{T})^{2}\left(1+\operatorname{reg}(\mathcal{T})^{2}\right)$. Lemma 2.3, Definitions 2.9 and 2.12 imply that

$$
\forall \mathcal{D} \in \mathfrak{D}, \quad \underline{\Delta}^{\mathcal{D}} p^{\mathfrak{Q}}=\Delta^{\mathcal{D}}\left(p^{\mathfrak{D}}\right)+\Delta_{\alpha}^{\mathcal{D}}\left(\mathrm{D}^{\mathfrak{D}} \mathbf{u}^{\mathcal{T}}\right) .
$$

3. DDFV schemes for the Stokes equation. The principle to get the modified DDFV scheme is the following: we integrate the momentum conservation law of the problem (1.1) on the interior primal mesh $\mathfrak{M}$ and the interior dual mesh $\mathfrak{M}^{*}$. The mass conservation equation is directly approached on the diamond mesh using the discrete operator $\operatorname{div}^{\mathfrak{D}}$ and the new stabilization term. We impose on $\partial \mathfrak{M}$ and on $\partial \mathfrak{M}^{*}$ the Dirichlet boundary conditions. Finally, the integral of the pressure is imposed to be equal to zero. The differences with the scheme (1.4) introduced in [21] are in the viscous stress tensor and the stabilization term, which takes now into account the jumps of the viscosity and the pressure. We replace $\eta^{\mathfrak{D}} \mathrm{D}^{\mathfrak{D}} \mathbf{u}^{\mathcal{T}}$ (resp. $-\lambda h_{\mathfrak{D}}^{2} \Delta^{\mathfrak{D}} p^{\mathfrak{D}}$ ) by $\mathrm{D}_{\mathfrak{D}}^{\eta, \mathcal{N}} \mathbf{u}^{\mathcal{T}}$ (resp. $-\lambda h_{\mathfrak{D}}^{2} \Delta^{\mathfrak{D}} p^{\mathfrak{Q}}$ ) as follows:

$$
\begin{cases}\text { Find } \mathbf{u}^{\mathcal{T}} \in \mathbb{E}_{0} \text { and } p^{\mathfrak{D}} \in \mathbb{R}^{\mathfrak{D}} \text { such that, } & \\ \operatorname{div}^{\mathfrak{M}}\left(-2 \mathrm{D}_{\mathfrak{D}}^{\eta, \mathcal{N}} \mathbf{u}^{\mathcal{T}}+p^{\mathfrak{D}} \mathrm{Id}\right)=\mathbf{f}^{\mathfrak{M}}, & \operatorname{div}^{\mathfrak{M} *}\left(-2 \mathrm{D}_{\mathfrak{D}}^{\eta, \mathcal{N}} \mathbf{u}^{\mathcal{T}}+p^{\mathfrak{D}} \mathrm{Id}\right)=\mathbf{f}^{\mathfrak{M} *}, \\ \operatorname{div}^{\mathfrak{D}}\left(\mathbf{u}^{\mathcal{T}}\right)-\lambda h_{\mathfrak{D}}^{2} \underline{\Delta}^{\mathfrak{D}} p^{\mathfrak{Q}}=0, & \sum_{\mathcal{D} \in \mathfrak{D}} m_{\mathcal{D}} p^{\mathcal{D}}=0,\end{cases}
$$


where $\lambda>0$ is given, $\mathbf{f}^{\mathfrak{M}}=\widetilde{\mathbb{P}}_{m}^{\mathfrak{m}} \mathbf{f}$ and $\mathbf{f}^{\mathfrak{M}^{*}}=\widetilde{\mathbb{P}}_{m}^{\mathfrak{m} *} \mathbf{f}$ (the projections are defined by (2.4)), and for any $\mathcal{D} \in \mathfrak{D},\left(\delta^{\mathcal{D}}, p^{\mathfrak{Q}_{\mathcal{D}}}\right) \in \mathcal{M}_{n_{\mathcal{D}}, 2}(\mathbb{R}) \times \mathbb{R}^{n_{\mathcal{D}}}$ satisfying (2.15).

If we take the old stabilization term $-\lambda h_{\mathfrak{D}}^{2} \Delta^{\mathfrak{D}} p^{\mathfrak{D}}$ instead of $-\lambda h_{\mathfrak{D}}^{2} \Delta^{\mathfrak{D}} p^{\mathfrak{Q}}$, the scheme is still well-posed but we did not succeed in proving first order error estimates, since we have take into account the jumps of pressure. The numerical tests also bring out the difference of these two stabilization term and show that the new form of the stabilization term actually improves the results.

4. Results on discrete operators. In this section, we present several results on the discrete operators. In Section 4.1, we focus on the modified and standard discrete strain rate tensor. The main result is the discrete Korn inequality for the modified one (see Theorem 4.2). Its proof consists in using the discrete Korn inequality proved in [21] for the standard discrete strain rate tensor and Lemma 2.2 that can be seen as a local Korn inequality for the velocity unknowns. Then in Section 4.2, we rewrite the discrete Stokes formula and finally we sum up results of [21].

\subsection{Discrete strain rate tensor.}

4.1.1. Estimations of the discrete strain rate tensor. We recall results proved in [21], and extend them on the quarter diamond cells. The first one is a consequence of Remark 2.1.

Proposition 4.1. For all $\mathbf{u}^{\mathcal{T}} \in\left(\mathbb{R}^{2}\right)^{T}$, we get

$$
\left\|\mathrm{D}^{\mathcal{D}} \mathbf{u}^{\mathcal{T}}\right\|_{2} \leq\left\|\nabla^{\mathcal{D}} \mathbf{u}^{\mathcal{T}}\right\|_{2} \quad \text { and } \quad\left\|\mathrm{D}_{\mathfrak{Q}}^{\mathcal{N}} \mathbf{u}^{\mathcal{T}}\right\|_{2} \leq\left\|\nabla_{\mathfrak{Q}}^{\mathcal{N}} \mathbf{u}^{\mathcal{T}}\right\|_{2} .
$$

The discrete strain rate tensor and the modified one can be compared as follows.

Lemma 4.1. Assume that $\eta$ satisfies (1.2). There exists a constant $C_{4}>0$, depending only on $\underline{\mathrm{C}}_{\eta}$ and $\overline{\mathrm{C}}_{\eta}$, such that for all $\mathbf{u}^{\mathcal{T}} \in\left(\mathbb{R}^{2}\right)^{T}$ :

$$
\left\|\mathrm{D}^{\mathfrak{D}} \mathbf{u}^{\mathcal{T}}\right\|_{2} \leq\left\|\mathrm{D}_{\mathfrak{2}}^{\mathcal{N}} \mathbf{u}^{\mathcal{T}}\right\|_{2} \leq C_{4}\left\|\mathrm{D}^{\mathfrak{D}} \mathbf{u}^{\mathcal{T}}\right\|_{2} .
$$

Proof. First estimate. Let $\mathcal{D} \in \mathfrak{D}$. The estimate is just a consequence of property of the matrix $B_{\mathcal{Q}}$ that is $\sum_{\mathcal{Q} \in \mathfrak{Q}_{\mathcal{D}}} m_{\mathcal{Q}} B_{\mathcal{Q}}=0$. Then we have $\left\|\mathrm{D}_{\mathfrak{Q}}^{\mathcal{N}} \mathbf{u}^{\mathcal{T}}\right\|_{2}^{2}=\left\|\mathrm{D}^{\mathcal{D}} \mathbf{u}^{\mathcal{T}}\right\|_{2}^{2}+\frac{1}{4} \| B_{\mathcal{Q}} \delta^{\mathcal{D}}+$ ${ }^{t} \delta^{\mathcal{D} t} B_{\mathcal{Q}} \|_{2}^{2}$, which concludes the first estimate.

Second estimate. Let $\mathcal{D} \in \mathfrak{D}$. The equality 2.17 gives

$$
\sum_{\mathcal{Q} \in \mathfrak{Q}_{\mathcal{D}}} m_{\mathcal{Q}} \eta_{\mathcal{Q}}\left(2 \mathrm{D}^{\mathcal{D}} \mathbf{u}^{\mathcal{T}}+B_{\mathcal{Q}} \delta^{\mathcal{D}}+{ }^{t} \delta^{\mathcal{D} t} B_{\mathcal{Q}}: B_{\mathcal{Q}} \delta^{\mathcal{D}}+{ }^{t} \delta^{\mathcal{D} t} B_{\mathcal{Q}}\right)=0 .
$$

Definition 2.6 of $\mathrm{D}_{\mathcal{Q}}^{\mathcal{N}} \mathbf{u}^{\mathcal{T}}$ implies that

$$
\sum_{\mathcal{Q} \in \mathfrak{Q}_{\mathcal{D}}} m_{\mathcal{Q}} \eta_{\mathcal{Q}}\left\|\mathrm{D}_{\mathcal{Q}}^{\mathcal{N}} \mathbf{u}^{\mathcal{T}}\right\|_{\mathcal{F}}^{2}=\sum_{\mathcal{Q} \in \mathfrak{Q}_{\mathcal{D}}} m_{\mathcal{Q}} \eta_{\mathcal{Q}}\left(\mathrm{D}_{\mathcal{Q}}^{\mathcal{N}} \mathbf{u}^{\mathcal{T}}: \mathrm{D}^{\mathcal{D}} \mathbf{u}^{\mathcal{T}}\right)
$$

Thanks to the inequality (1.2), we get

$$
\underline{\mathrm{C}}_{\eta} \sum_{\mathcal{Q} \in \mathfrak{Q}_{\mathcal{D}}} m_{\mathcal{Q}}\left\|\mathrm{D}_{\mathcal{Q}}^{\mathcal{N}} \mathbf{u}^{\mathcal{T}}\right\|_{\mathcal{F}}^{2} \leq \overline{\mathrm{C}}_{\eta} \sum_{\mathcal{Q} \in \mathfrak{Q}_{\mathcal{D}}} m_{\mathcal{Q}}\left(\mathrm{D}_{\mathcal{Q}}^{\mathcal{N}} \mathbf{u}^{\mathcal{T}}: \mathrm{D}^{\mathcal{D}} \mathbf{u}^{\mathcal{T}}\right) .
$$

Applying Cauchy-Schwarz inequality, we obtain

$$
\underline{\mathrm{C}}_{\eta}^{2} \sum_{\mathcal{Q} \in \mathfrak{Q}_{\mathcal{D}}} m_{\mathcal{Q}}\left\|\mathrm{D}_{\mathcal{Q}}^{\mathcal{N}} \mathbf{u}^{\mathcal{T}}\right\|_{\mathcal{F}}^{2} \leq \overline{\mathrm{C}}_{\eta}^{2} m_{\mathcal{D}}\left\|\mathrm{D}^{\mathcal{D}} \mathbf{u}^{\mathcal{T}}\right\|_{\mathcal{F}}^{2} .
$$

Noting $C_{4}=\frac{\overline{\mathrm{C}}_{\eta}}{\underline{\mathrm{C}}_{\eta}}$, we get the result. 
4.1.2. Discrete Korn inequality. In this section, we recall the discrete Korn inequality which is already known and prove a new one for the modified operators.

THEOREM 4.1 (Discrete Korn inequality on diamond cells, [21, Theorem 5.1]). For all $\mathbf{u}^{\mathcal{T}} \in \mathbb{E}_{0}$, we have

$$
\left\|\nabla^{\mathfrak{D}} \mathbf{u}^{\mathcal{T}}\right\|_{2} \leq \sqrt{2}\left\|\mathrm{D}^{\mathfrak{D}} \mathbf{u}^{\mathcal{T}}\right\|_{2}
$$

THEOREM 4.2 (Discrete Korn inequality on quarter diamond cells). Assume that $\eta$ satisfies (1.2). There exists $C_{5}>0$ depending only on $\underline{\mathrm{C}}_{\eta}, \overline{\mathrm{C}}_{\eta}, \operatorname{reg}(\mathcal{T})$ and $\sin \left(\epsilon_{0}\right)$ such that:

$$
\left\|\nabla_{\mathfrak{Q}}^{\mathcal{N}} \mathbf{u}^{\mathcal{T}}\right\|_{2} \leq C_{5}\left\|\mathrm{D}_{\mathfrak{Q}}^{\mathcal{N}} \mathbf{u}^{\mathcal{T}}\right\|_{2}, \quad \forall \mathbf{u}^{\mathcal{T}} \in \mathbb{E}_{0}
$$

Proof. The equality (4.1) implies that

$$
\sum_{\mathcal{Q} \in \mathfrak{Q}_{\mathcal{D}}} m_{\mathcal{Q}} \eta_{\mathcal{Q}}\left\|B_{\mathcal{Q}} \delta^{\mathcal{D}}+{ }^{t} \delta^{\mathcal{D} t} B_{\mathcal{Q}}\right\|_{\mathcal{F}}^{2}=-\sum_{\mathcal{Q} \in \mathfrak{Q}_{\mathcal{D}}} m_{\mathcal{Q}} \eta_{\mathcal{Q}}\left(2 \mathrm{D}^{\mathcal{D}} \mathbf{u}^{\mathcal{T}}: B_{\mathcal{Q}} \delta^{\mathcal{D}}+{ }^{t} \delta^{\mathcal{D} t} B_{\mathcal{Q}}\right) .
$$

Cauchy-Schwarz inequality and (1.2) imply that

$$
\begin{aligned}
& \underline{\mathrm{C}}_{\eta} \sum_{\mathcal{Q} \in \mathfrak{Q}_{\mathcal{D}}} m_{\mathcal{Q}}\left\|B_{\mathcal{Q}} \delta^{\mathcal{D}}+{ }^{t} \delta^{\mathcal{D} t} B_{\mathcal{Q}}\right\|_{\mathcal{F}}^{2} \\
& \quad \leq \overline{\mathrm{C}}_{\eta}\left(m_{\mathcal{D}}\left\|\mathrm{D}^{\mathcal{D}} \mathbf{u}^{\mathcal{T}}\right\|_{\mathcal{F}}^{2}\right)^{\frac{1}{2}}\left(\sum_{\mathcal{Q} \in \mathfrak{Q}_{\mathcal{D}}} m_{\mathcal{Q}}\left\|B_{\mathcal{Q}} \delta^{\mathcal{D}}+{ }^{t} \delta^{\mathcal{D} t} B_{\mathcal{Q}}\right\|_{\mathcal{F}}^{2}\right)^{\frac{1}{2}} .
\end{aligned}
$$

It follows that

$$
\sum_{\mathcal{Q} \in \mathfrak{Q}_{\mathcal{D}}} m_{\mathcal{Q}}\left\|B_{\mathcal{Q}} \delta^{\mathcal{D}}+{ }^{t} \delta^{\mathcal{D} t} B_{\mathcal{Q}}\right\|_{\mathcal{F}}^{2} \leq \frac{\overline{\mathrm{C}}_{\eta}^{2}}{\underline{\mathrm{C}}_{\eta}^{2}} m_{\mathcal{D}}\left\|\mathrm{D}^{\mathcal{D}} \mathbf{u}^{\mathcal{T}}\right\|_{\mathcal{F}}^{2}
$$

Thanks to Lemma 2.2, we deduce

$$
\sum_{\mathcal{Q} \in \mathfrak{Q}_{\mathcal{D}}} m_{\mathcal{Q}}\left\|B_{\mathcal{Q}} \delta^{\mathcal{D}}\right\|_{\mathcal{F}}^{2} \leq C_{1} \frac{\overline{\mathrm{C}}_{\eta}^{2}}{\underline{\mathrm{C}}_{\eta}^{2}} m_{\mathcal{D}}\left\|\mathrm{D}^{\mathcal{D}} \mathbf{u}^{\mathcal{T}}\right\|_{\mathcal{F}}^{2}
$$

Furthermore, it gives

$$
\sum_{\mathcal{Q} \in \mathfrak{Q}_{\mathcal{D}}} m_{\mathcal{Q}}\left\|\nabla_{\mathcal{Q}}^{\mathcal{N}} \mathbf{u}^{\mathcal{T}}\right\|_{\mathcal{F}}^{2} \leq 2 m_{\mathcal{D}}\left\|\nabla^{\mathcal{D}} \mathbf{u}^{\mathcal{T}}\right\|_{\mathcal{F}}^{2}+2 C_{1} \frac{\overline{\mathrm{C}}_{\eta}^{2}}{\underline{\mathrm{C}}_{\eta}^{2}} m_{\mathcal{D}}\left\|\mathrm{D}^{\mathcal{D}} \mathbf{u}^{\mathcal{T}}\right\|_{\mathcal{F}}^{2} .
$$

Using the discrete Korn inequality Theorem 4.1 and than Lemma 4.1, we conclude

$$
\left\|\nabla_{\mathfrak{Q}}^{\mathcal{N}} \mathbf{u}^{\mathcal{T}}\right\|_{2}^{2} \leq 4\left(1+C_{1} \frac{\overline{\mathrm{C}}_{\eta}^{2}}{\underline{\mathrm{C}}_{\eta}^{2}}\right)\left\|\mathrm{D}^{\mathfrak{D}} \mathbf{u}^{\mathcal{T}}\right\|_{2}^{2} \leq 4\left(1+C_{1} \frac{\overline{\mathrm{C}}_{\eta}^{2}}{\underline{\mathrm{C}}_{\eta}^{2}}\right)\left\|\mathrm{D}_{\mathfrak{Q}}^{\mathcal{N}} \mathbf{u}^{\mathcal{T}}\right\|_{2}^{2} .
$$

Using Lemma 4.1, these two discrete Korn inequalities allow us to compare the discrete gradient and the modified one, as follows. It does not seem possible to show this result directly, that is without using Korn inequalities.

LEMMA 4.2. Assume that $\eta$ satisfies (1.2). There exists a constant $C_{6}>0$, depending only on $\underline{\mathrm{C}}_{\eta}, \overline{\mathrm{C}}_{\eta}, \operatorname{reg}(\mathcal{T})$ and $\sin \left(\epsilon_{0}\right)$ such that for all $\mathbf{u}^{\mathcal{T}} \in \mathbb{E}_{0}$ :

$$
\left\|\nabla^{\mathfrak{D}} \mathbf{u}^{\mathcal{T}}\right\|_{2} \leq\left\|\nabla_{\mathfrak{Q}}^{\mathcal{N}} \mathbf{u}^{\mathcal{T}}\right\|_{2} \leq C_{6}\left\|\nabla^{\mathfrak{D}} \mathbf{u}^{\mathcal{T}}\right\|_{2} .
$$


4.2. Discrete Stokes formula. The discrete gradient and discrete divergence for a vectorvalued function are known to satisfy a discrete Stokes formula, as follows.

THEOREM 4.3 (Discrete Stokes formula [21, Theorem 3.1]). We have

$$
\llbracket \operatorname{div}^{\mathcal{T}} \xi^{\mathfrak{D}}, \mathbf{v}^{\mathcal{T}} \rrbracket_{\mathcal{T}}=-\left(\xi^{\mathfrak{D}}: \nabla^{\mathfrak{D}} \mathbf{v}^{\mathcal{T}}\right)_{\mathfrak{D}}, \quad \forall\left(\xi^{\mathfrak{D}}, \mathbf{v}^{\mathcal{T}}\right) \in\left(\mathcal{M}_{2}(\mathbb{R})\right)^{\mathfrak{D}} \times \mathbb{E}_{0} .
$$

Since we have introduced modified discrete operators on the quarter diamond cells, we want to rewrite the discrete Stokes formula for the specific tensor $\mathrm{D}_{\mathfrak{D}}^{\eta, \mathcal{N}} \mathbf{u}^{\mathcal{T}}$ (see Definition 2.7).

THEOREM 4.4. We have, for all $\left(\mathbf{u}^{\mathcal{T}}, \mathbf{v}^{\mathcal{T}}\right) \in\left(\mathbb{R}^{2}\right)^{\mathcal{T}} \times \mathbb{E}_{0}$

$$
\llbracket \operatorname{div}^{\mathcal{T}}\left(\mathrm{D}_{\mathfrak{D}}^{\eta, \mathcal{N}} \mathbf{u}^{\mathcal{T}}\right), \mathbf{v}^{\mathcal{T}} \rrbracket_{\mathcal{T}}=-\left(\eta^{\mathfrak{Q}} \mathrm{D}_{\mathfrak{Q}}^{\mathcal{N}} \mathbf{u}^{\mathcal{T}}: \nabla_{\mathfrak{Q}}^{\mathcal{N}} \mathbf{v}^{\mathcal{T}}\right)_{\mathfrak{Q}}
$$

Proof. The first discrete Stokes formula 4.3 gives

$\llbracket \operatorname{div}^{\mathcal{T}}\left(\mathrm{D}_{\mathfrak{D}}^{\eta, \mathcal{N}} \mathbf{u}^{\mathcal{T}}\right), \mathbf{v}^{\mathcal{T}} \rrbracket_{\mathcal{T}}=-\left(\mathrm{D}_{\mathfrak{D}}^{\eta, \mathcal{N}} \mathbf{u}^{\mathcal{T}}: \nabla^{\mathfrak{D}} \mathbf{v}^{\mathcal{T}}\right)_{\mathfrak{D}}=-\sum_{\mathcal{D} \in \mathfrak{D}} \sum_{\mathcal{Q} \in \mathfrak{Q}_{\mathcal{D}}} m_{\mathcal{Q}} \eta_{\mathcal{Q}}\left(\mathrm{D}_{\mathcal{Q}}^{\mathcal{N}} \mathbf{u}^{\mathcal{T}}: \nabla^{\mathcal{D}} \mathbf{v}^{\mathcal{T}}\right)$.

Thanks to Theorem 2.1, there exists a unique $\left(\widehat{\delta^{\mathcal{D}}}, \widehat{p^{\mathfrak{Q D}^{\mathcal{D}}}}\right) \in \mathcal{M}_{n_{\mathcal{D}}, 2}(\mathbb{R}) \times \mathbb{R}^{n_{\mathcal{D}}}$ satisfying equations (2.15), with $\mathrm{D}^{\mathcal{D}} \mathbf{v}^{\mathcal{T}}$ and $p^{\mathfrak{D}}$. Using the symmetry of $\mathrm{D}^{\mathcal{D}} \mathbf{v}^{\mathcal{T}}$, we have

$$
\left(\mathrm{D}_{\mathfrak{D}}^{\eta, \mathcal{N}} \mathbf{u}^{\mathcal{T}}: \nabla^{\mathfrak{D}} \mathbf{v}^{\mathcal{T}}\right)_{\mathfrak{D}}=\sum_{\mathcal{D} \in \mathfrak{D}} \sum_{\mathcal{Q} \in \mathfrak{Q}_{\mathcal{D}}} m_{\mathcal{Q}} \eta_{\mathcal{Q}}\left(\mathrm{D}_{\mathcal{Q}}^{\mathcal{N}} \mathbf{u}^{\mathcal{T}}: \mathrm{D}_{\mathcal{Q}}^{\mathcal{N}} \mathbf{v}^{\mathcal{T}}-\frac{1}{2}\left({ }^{t} \widehat{\delta^{\mathcal{D}}} t B_{\mathcal{Q}}-B_{\mathcal{Q}} \widehat{\delta^{\mathcal{D}}}\right)\right) .
$$

Right-multiplying (2.15a) by $\widehat{\delta^{\mathcal{D}}}$ and applying the trace operator, we get

$$
\sum_{\mathcal{Q} \in \mathfrak{Q}_{\mathcal{D}}} m_{\mathcal{Q}}(\underbrace{2 \eta_{\mathcal{Q}} \mathrm{D}^{\mathcal{D}} \mathbf{u}^{\mathcal{T}}+\eta_{\mathcal{Q}}\left(B_{\mathcal{Q}} \delta^{\mathcal{D}}+{ }^{t} \delta^{\mathcal{D} t} B_{\mathcal{Q}}\right)-p^{\mathcal{Q}} \mathrm{Id}}_{=\varphi_{\mathcal{Q}}\left(\mathrm{D}^{\mathcal{D}} \mathbf{u}^{\mathcal{T}}, \delta^{\mathcal{D}}, p^{\mathfrak{Q} \mathcal{D}}\right)}: B_{\mathcal{Q}} \widehat{\delta^{\mathcal{D}}}+{ }^{t} \widehat{\delta^{\mathcal{D}} t} B_{\mathcal{Q}})=0
$$

since $\varphi_{\mathcal{Q}}\left(\mathrm{D}^{\mathcal{D}} \mathbf{u}^{\mathcal{T}}, \delta^{\mathcal{D}}, p^{\mathcal{Q}}\right)$ is a symmetric matrix. Furthermore since we have (Id : $B_{\mathcal{Q}} \widehat{\delta^{\mathcal{D}}}+$ $\left.{ }^{t} \widehat{\delta^{\mathcal{D}}} t B_{\mathcal{Q}}\right)=\operatorname{Tr}\left(B_{\mathcal{Q}} \widehat{\delta^{\mathcal{D}}}\right)=0$ by $(2.15$ b), we obtain

$$
\sum_{\mathcal{Q} \in \mathfrak{Q}_{\mathcal{D}}} m_{\mathcal{Q}} \eta_{\mathcal{Q}}(\underbrace{2 \mathrm{D}^{\mathcal{D}} \mathbf{u}^{\mathcal{T}}+B_{\mathcal{Q}} \delta^{\mathcal{D}}+{ }^{t} \delta^{\mathcal{D} t} B_{\mathcal{Q}}}_{=2 \mathrm{D}_{\mathcal{Q}}^{\mathcal{N}} \mathbf{u}^{\mathcal{T}}}:{ }^{t} \widehat{\delta}^{\underline{\mathcal{D}}} B_{\mathcal{Q}}+B_{\mathcal{Q}} \widehat{\delta^{\mathcal{D}}})=0
$$

Substituting this equality in (4.3), we deduce that

$$
\left(\mathrm{D}_{\mathfrak{D}}^{\eta, \mathcal{N}} \mathbf{u}^{\mathcal{T}}: \nabla^{\mathfrak{D}} \mathbf{v}^{\mathcal{T}}\right)_{\mathfrak{D}}=\sum_{\mathcal{D} \in \mathfrak{D}} \sum_{\mathcal{Q} \in \mathfrak{Q}_{\mathcal{D}}} m_{\mathcal{Q}} \eta_{\mathcal{Q}}\left(\mathrm{D}_{\mathcal{Q}}^{\mathcal{N}} \mathbf{u}^{\mathcal{T}}: \mathrm{D}_{\mathcal{Q}}^{\mathcal{N}} \mathbf{v}^{\mathcal{T}}\right)
$$

The symmetry of $\mathrm{D}_{\mathcal{Q}}^{\mathcal{N}} \mathbf{u}^{\mathcal{T}}$ implies the result.

4.3. Poincaré inequality. Properties of the mean-value projection operator. We recall results already known in the literature.

THEOREM 4.5 (Discrete Poincaré inequality [21, Theorem 5.2]). Let $\mathcal{T}$ be a mesh of $\Omega$. There exists a constant $C_{7}>0$, depending only on the diameter of $\Omega$ and $\operatorname{reg}(\mathcal{T})$, such that

$$
\left\|\mathbf{u}^{\mathcal{T}}\right\|_{2} \leq C_{7}\left\|\nabla^{\mathfrak{D}} \mathbf{u}^{\mathcal{T}}\right\|_{2}, \quad \forall \mathbf{u}^{\mathcal{T}} \in \mathbb{E}_{0} .
$$

Lemma 4.3 ([21, Lemma 5.5, Proposition 5.5]). Let $\mathcal{T}$ be a mesh of $\Omega$. There exists $C_{8}, C_{9}>0$ depending only on $\operatorname{reg}(\mathcal{T})$, such that for any function $\mathbf{v}$ in $\left(H_{0}^{1}(\Omega)\right)^{2}$, we have

$$
\left\|\nabla^{\mathfrak{D}} \mathbb{P}_{\boldsymbol{m}}^{\mathcal{T}} \mathbf{v}\right\|_{2} \leq C_{8}\|\nabla \mathbf{v}\|_{2} \text { and } \sum_{\mathcal{D} \in \mathfrak{D}} \int_{\mathcal{D}} p^{\mathcal{D}}\left(\operatorname{div}^{\mathcal{D}}\left(\mathbb{P}_{\boldsymbol{m}}^{\mathcal{T}} \mathbf{v}\right)-\operatorname{div}(\mathbf{v})\right) \mathrm{d} z \leq C_{9}\left|p^{\mathfrak{D}}\right|_{h}\|\mathbf{v}\|_{H^{1}}
$$


LEMMA 4.4 ([21, Lemma 3.1]). Let $\mathcal{T}$ be a mesh of $\Omega$. There exists $C_{10}>0$ depending only on $\operatorname{reg}(\mathcal{T})$, such that for any $p^{\mathfrak{D}} \in \mathbb{R}^{\mathfrak{D}}$, we have

$$
\left|p^{\mathfrak{D}}\right|_{h} \leq C_{10}\left\|p^{\mathfrak{D}}\right\|_{2}
$$

LEMMA 4.5 ([1, Lemma 3.4]). There exists a number $C_{11}>0$ such that for any bounded set $\mathcal{P} \subset \mathbb{R}^{2}$ with positive measure, any segment $\sigma \subset \mathbb{R}^{2}$ and any $v \in H^{1}\left(\mathbb{R}^{2}\right)$, we have

$$
\left|v_{\mathcal{P}}-v_{\sigma}\right|^{2} \leq \frac{1}{m_{\sigma} m_{\mathcal{P}}} \int_{\sigma} \int_{\mathcal{P}}|v(x)-v(y)|^{2} \mathrm{~d} x \mathrm{~d} y \leq C_{11} \frac{\operatorname{diam}\left(\widehat{\mathcal{P}_{\sigma}}\right)^{3}}{m_{\sigma} m_{\mathcal{P}}} \int_{\widehat{\mathcal{P}_{\sigma}}}|\nabla v(z)|^{2} \mathrm{~d} z,
$$

where $v_{\mathcal{P}}$ denotes the mean value of $v$ on $\mathcal{P}, v_{\sigma}$ the mean value of $v$ on the segment $\sigma$, and $\widehat{\mathcal{P}_{\sigma}}$ is the convex hull of $\mathcal{P} \cup \sigma$.

5. Stability of the scheme. In this section, we prove the well-posedness and the uniform stability of our finite volume scheme. The proof of the uniform stability result relies on an appropriate choice of the stabilization term.

DEFINITION 5.1. We define the bilinear form associated to our DDFV scheme (3.1):

$\forall\left(\mathbf{u}^{\mathcal{T}}, p^{\mathfrak{D}}\right),\left(\widetilde{\mathbf{u}}^{\mathcal{T}}, \widetilde{p}^{\mathfrak{D}}\right) \in\left(\mathbb{R}^{2}\right)^{\mathcal{T}} \times \mathbb{R}^{\mathfrak{D}}$,

$B\left(\mathbf{u}^{\mathcal{T}}, p^{\mathfrak{D}} ; \widetilde{\mathbf{u}}^{\mathcal{T}}, \widetilde{p}^{\mathfrak{D}}\right)=\llbracket \operatorname{div}^{\mathcal{T}}\left(-2 \mathrm{D}_{\mathfrak{D}}^{\eta, \mathcal{N}} \mathbf{u}^{\mathcal{T}}+p^{\mathfrak{D}} \mathrm{Id}\right), \widetilde{\mathbf{u}}^{\mathcal{T}} \rrbracket_{\mathcal{T}}+\left(\operatorname{div}^{\mathfrak{D}}\left(\mathbf{u}^{\mathcal{T}}\right)-\lambda h_{\mathfrak{D}}^{2} \underline{\Delta}^{\mathfrak{D}}\left(p^{\mathfrak{Q}}\right), \widetilde{p}^{\mathfrak{D}}\right)_{\mathfrak{D}}$,

where $\lambda>0$ and $\left(\delta^{\mathfrak{D}}, p^{\mathfrak{Q}}\right)$ is the solution of (2.15) for $\mathrm{D}^{\mathfrak{D}} \mathbf{u}^{\mathcal{T}}$ and $p^{\mathfrak{D}}$.

THEOREM 5.1 (Stability of the scheme). Assume that $\eta$ satisfies (1.2) and $\lambda<\frac{4 \underline{\mathrm{C}}_{\eta}}{C_{3}}$. Then there exists $C_{12}, C_{13}>0$, depending only on the diameter of $\Omega, \lambda, \underline{\mathrm{C}}_{\eta}, \overline{\mathrm{C}}_{\eta}, \operatorname{reg}(\mathcal{T})$ and $\sin \left(\epsilon_{0}\right)$, such that for each pair $\left(\mathbf{u}^{\mathcal{T}}, p^{\mathfrak{D}}\right) \in \mathbb{E}_{0} \times \mathbb{R}^{\mathfrak{D}}$ such that $\sum_{\mathcal{D} \in \mathfrak{D}} m_{\mathcal{D}} p^{\mathcal{D}}=0$, there exists $\left(\widetilde{\mathbf{u}}^{\mathcal{T}}, \widetilde{p}^{\mathfrak{D}}\right) \in \mathbb{E}_{0} \times \mathbb{R}^{\mathfrak{D}}$ with:

$$
\left\|\nabla_{\mathfrak{Q}}^{\mathcal{N}} \widetilde{\mathbf{u}}^{\mathcal{T}}\right\|_{2}+\left\|\widetilde{p}^{\mathfrak{Q}}\right\|_{2} \leq C_{12}\left(\left\|\nabla_{\mathfrak{Q}}^{\mathcal{N}} \mathbf{u}^{\mathcal{T}}\right\|_{2}+\left\|p^{\mathfrak{Q}}\right\|_{2}\right)
$$

and

$$
\left\|\nabla_{\mathfrak{Q}}^{\mathcal{N}} \mathbf{u}^{\mathcal{T}}\right\|_{2}^{2}+\left\|p^{\mathfrak{Q}}\right\|_{2}^{2} \leq C_{13} B\left(\mathbf{u}^{\mathcal{T}}, p^{\mathfrak{D}} ; \widetilde{\mathbf{u}}^{\mathcal{T}}, \widetilde{p}^{\mathfrak{D}}\right) .
$$

with $\left(\delta^{\mathfrak{D}}, p^{\mathfrak{Q}}\right)$ (resp. $\left.\left(\widetilde{\delta}^{\mathfrak{D}}, \widetilde{p}^{\mathfrak{Q}}\right)\right)$ is the solution of $(2.15)$ for $\mathrm{D}^{\mathfrak{D}} \mathbf{u}^{\mathcal{T}}$ and $p^{\mathfrak{D}}$ (resp. $\mathrm{D}^{\mathfrak{D}} \widetilde{\mathbf{u}}^{\mathcal{T}}$ and $\left.\widetilde{p}^{\mathfrak{D}}\right)$, thus we have $m_{\mathcal{D}} p^{\mathcal{D}}=\sum_{\mathcal{Q} \in \mathfrak{Q}_{\mathcal{D}}} m_{\mathcal{Q}} p^{\mathcal{Q}}$, for all $\mathcal{D} \in \mathfrak{D}$.

The technical condition $\lambda<\frac{4 \underline{\mathrm{C}}_{\eta}}{C_{3}}$ does not seem to be mandatory for the scheme to be stable. In practice, we did not find positive values of $\lambda$ leading to instabilities.

Proof. Let $\left(\mathbf{u}^{\mathcal{T}}, p^{\mathfrak{D}}\right) \in \mathbb{E}_{0} \times \mathbb{R}^{\mathfrak{D}}$ such that $\sum_{\mathcal{D} \in \mathfrak{D}} m_{\mathcal{D}} p^{\mathcal{D}}=0$. The proof of this Theorem is obtained by building explicitly $\left(\widetilde{\mathbf{u}}^{\mathcal{T}}, \widetilde{p}^{\mathfrak{D}}\right) \in \mathbb{E}_{0} \times \mathbb{R}^{\mathfrak{D}}$ such that (5.1) and (5.2) hold.

Step 1 . We apply to $B$ the two discrete Stokes formula Theorem 4.3 and Theorem 4.4:

$$
B\left(\mathbf{u}^{\mathcal{T}}, p^{\mathfrak{D}} ; \mathbf{u}^{\mathcal{T}}, p^{\mathfrak{D}}\right)=\left(2 \eta^{\mathfrak{Q}} \mathrm{D}_{\mathfrak{Q}}^{\mathcal{N}} \mathbf{u}^{\mathcal{T}}: \nabla_{\mathfrak{Q}}^{\mathcal{N}} \mathbf{u}^{\mathcal{T}}\right)_{\mathfrak{Q}}-\left(\lambda h_{\mathfrak{D}}^{2} \underline{\Delta}^{\mathfrak{D}}\left(p^{\mathfrak{Q}}\right), p^{\mathfrak{D}}\right)_{\mathfrak{D}} .
$$

The symmetry of $\mathrm{D}_{\mathfrak{Q}}^{\mathcal{N}} \mathbf{u}^{\mathcal{T}}$ and (2.22) imply that

$$
B\left(\mathbf{u}^{\mathcal{T}}, p^{\mathfrak{D}} ; \mathbf{u}^{\mathcal{T}}, p^{\mathfrak{D}}\right)=\left(2 \eta^{\mathfrak{Q}} \mathrm{D}_{\mathfrak{Q}}^{\mathcal{N}} \mathbf{u}^{\mathcal{T}}: \mathrm{D}_{\mathfrak{Q}}^{\mathcal{N}} \mathbf{u}^{\mathcal{T}}\right)_{\mathfrak{Q}}-\left(\lambda h_{\mathfrak{D}}^{2} \Delta^{\mathfrak{D}}\left(p^{\mathfrak{D}}\right)+\lambda h_{\mathfrak{D}}^{2} \Delta_{\alpha}^{\mathfrak{D}}\left(\mathrm{D}^{\mathfrak{D}} \mathbf{u}^{\mathcal{T}}\right), p^{\mathfrak{D}}\right)_{\mathfrak{D}}
$$


Reorganizing the sum over all the sides $\mathfrak{s} \in \mathfrak{S}$ of all the diamond cells, we have

$$
-\left(h_{\mathfrak{D}}^{2} \Delta_{\alpha}^{\mathfrak{D}}\left(\mathrm{D}^{\mathfrak{D}} \mathbf{u}^{\mathcal{T}}\right), p^{\mathfrak{D}}\right)_{\mathfrak{D}}=\sum_{\mathfrak{s}=\mathcal{D} \mid \mathcal{D}^{\prime} \in \mathfrak{S}}\left(h_{\mathcal{D}}^{2}+h_{\mathcal{D}^{\prime}}^{2}\right)\left(\alpha_{\mathfrak{s}, \mathcal{D}^{\prime}}\left(\mathrm{D}^{\mathcal{D}^{\prime}} \mathbf{u}^{\mathcal{T}}\right)-\alpha_{\mathfrak{s}, \mathcal{D}}\left(\mathrm{D}^{\mathcal{D}} \mathbf{u}^{\mathcal{T}}\right)\right)\left(p^{\mathcal{D}^{\prime}}-p^{\mathcal{D}}\right) .
$$

Young inequality and (2.20) imply that

$$
-\lambda\left(h_{\mathfrak{D}}^{2} \Delta^{\mathfrak{D}}\left(p^{\mathfrak{D}}\right)+h_{\mathfrak{D}}^{2} \Delta_{\alpha}^{\mathfrak{D}}\left(\mathrm{D}^{\mathfrak{D}} \mathbf{u}^{\mathcal{T}}\right), p^{\mathfrak{D}}\right)_{\mathfrak{D}} \geq \frac{\lambda}{2}\left|p^{\mathfrak{D}}\right|_{h}^{2}-\frac{\lambda}{2}\left|\mathrm{D}^{\mathfrak{D}} \mathbf{u}^{\mathcal{T}}\right|_{\alpha, h}^{2}
$$

Thanks to the inequality (1.2), we obtain

$$
B\left(\mathbf{u}^{\mathcal{T}}, p^{\mathfrak{D}} ; \mathbf{u}^{\mathcal{T}}, p^{\mathfrak{D}}\right) \geq 2 \underline{\mathrm{C}}_{\eta}\left\|\mathrm{D}_{\mathfrak{Z}}^{\mathcal{N}} \mathbf{u}^{\mathcal{T}}\right\|_{2}^{2}+\frac{\lambda}{2}\left|p^{\mathfrak{D}}\right|_{h}^{2}-\frac{\lambda}{2}\left|\mathrm{D}^{\mathfrak{D}} \mathbf{u}^{\mathcal{T}}\right|_{\alpha, h}^{2}
$$

Thanks to (2.21) and Lemma 4.1, we have $\left|\mathrm{D}^{\mathfrak{D}} \mathbf{u}^{\mathcal{T}}\right|_{\alpha, h}^{2} \leq C_{3}\left\|\mathrm{D}_{\mathfrak{Q}}^{\mathcal{N}} \mathbf{u}^{\mathcal{T}}\right\|_{2}^{2}$. Finally we use the discrete Korn inequality on quarter diamond cells (Theorem 4.2) in order to get

$$
B\left(\mathbf{u}^{\mathcal{T}}, p^{\mathfrak{D}} ; \mathbf{u}^{\mathcal{T}}, p^{\mathfrak{D}}\right) \geq \frac{1}{C_{5}^{2}}\left(2 \underline{\mathrm{C}}_{\eta}-C_{3} \frac{\lambda}{2}\right)\left\|\nabla_{\mathfrak{Q}}^{\mathcal{N}} \mathbf{u}^{\mathcal{T}}\right\|_{2}^{2}+\frac{\lambda}{2}\left|p^{\mathfrak{D}}\right|_{h}^{2}
$$

With $\lambda<\frac{4 \underline{\mathrm{C}}_{\eta}}{C_{3}}$, the constants in the above estimate are positive. Note that the above estimate on the pressure is mesh dependent (the semi-norm $|\cdot|_{h}$ is itself mesh dependent). That is why we could not bound uniformly the $L^{2}(\Omega)$-norm of the pressure by the semi-norm $|.|_{h}$.

Step 2. We use the Nečas Lemma (see [16, Corollary 2.4] or [4, Lemma III.1.17]): since $p^{\mathfrak{Q}}=\sum_{\mathcal{D} \in \mathfrak{D}} \sum_{\mathcal{Q} \in \mathfrak{Q}_{\mathcal{D}}} p^{\mathcal{Q}} \mathbf{1}_{\mathcal{Q}} \in L^{2}(\Omega)$ and its integral over $\Omega$ is zero, there exists a constant $C>0$ depending only on $\Omega$, and $\mathbf{v} \in\left(H_{0}^{1}(\Omega)\right)^{2}$ such that $\operatorname{div}(\mathbf{v})=-p^{\mathfrak{Q}}$ and

$$
\|\mathbf{v}\|_{H^{1}} \leq C\left\|p^{\mathfrak{Q}}\right\|_{2} .
$$

Let us choose $\mathbf{v}^{\mathcal{T}}=\mathbb{P}_{\boldsymbol{m}}^{\mathcal{T}} \mathbf{v}$ the mean-value projection $\mathbb{P}_{\boldsymbol{m}}^{\mathcal{T}} \mathbf{v}$, defined by (2.5). In particular, we have $\mathbf{v}^{\mathcal{T}} \in \mathbb{E}_{0}$. Thanks to Lemma 4.1, Proposition 4.1 and Lemma 4.3, we deduce

$$
\left\|\mathrm{D}_{\mathfrak{Q}}^{\mathcal{N}} \mathbf{v}^{\mathcal{T}}\right\|_{2} \leq C_{4} C_{8} C\left\|p^{\mathfrak{Q}}\right\|_{2}
$$

Theorem 4.4 implies

$$
B\left(\mathbf{u}^{\mathcal{T}}, p^{\mathfrak{D}} ; \mathbf{v}^{\mathcal{T}}, 0\right)=2\left(\eta^{\mathfrak{Q}} \mathrm{D}_{\mathfrak{Q}}^{\mathcal{N}} \mathbf{u}^{\mathcal{T}}: \nabla_{\mathfrak{Q}}^{\mathcal{N}} \mathbf{v}^{\mathcal{T}}\right)_{\mathfrak{Q}}-\left(p^{\mathfrak{D}}, \operatorname{div}^{\mathfrak{D}}\left(\mathbf{v}^{\mathcal{T}}\right)\right)_{\mathfrak{D}}
$$

Using the fact that $\left(\eta^{\mathfrak{Q}} \mathrm{D}_{\mathfrak{Q}}^{\mathcal{N}} \mathbf{u}^{\mathcal{T}}: \nabla_{\mathfrak{Q}}^{\mathcal{N}} \mathbf{v}^{\mathcal{T}}\right)_{\mathfrak{Q}}=\left(\eta^{\mathfrak{Q}} \nabla_{\mathfrak{Q}}^{\mathcal{N}} \mathbf{u}^{\mathcal{T}}: \mathrm{D}_{\mathfrak{Q}}^{\mathcal{N}} \mathbf{v}^{\mathcal{T}}\right)_{\mathfrak{Q}}$ and the CauchySchwarz inequality, we deduce

$$
\begin{aligned}
B\left(\mathbf{u}^{\mathcal{T}}, p^{\mathfrak{D}} ; \mathbf{v}^{\mathcal{T}}, 0\right) \geq-\overline{\mathrm{C}}_{\eta}\left\|\nabla_{\mathfrak{Q}}^{\mathcal{N}} \mathbf{u}^{\mathcal{T}}\right\|_{2}\left\|\mathrm{D}_{\mathfrak{Q}}^{\mathcal{N}} \mathbf{v}^{\mathcal{T}}\right\|_{2}-\sum_{\mathcal{D} \in \mathfrak{D}} \sum_{\mathcal{Q} \in \mathfrak{Q}_{\mathcal{D}}} \int_{\mathcal{Q}} p^{\mathcal{Q}} \operatorname{div}(\mathbf{v}(z)) \mathrm{d} z \\
\quad-\sum_{\mathcal{D} \in \mathfrak{D}} \int_{\mathcal{D}} p^{\mathcal{D}}\left(\operatorname{div}^{\mathcal{D}}\left(\mathbf{v}^{\mathcal{T}}\right)-\operatorname{div}(\mathbf{v}(z))\right) \mathrm{d} z+\sum_{\mathcal{D} \in \mathfrak{D}} \sum_{\mathcal{Q} \in \mathfrak{Q}_{\mathcal{D}}} \int_{\mathcal{Q}}\left(p^{\mathcal{Q}}-p^{\mathcal{D}}\right) \operatorname{div}(\mathbf{v}(z)) \mathrm{d} z .
\end{aligned}
$$

Since we have $\operatorname{div}(\mathbf{v})=-p^{\mathfrak{Q}}$ and the inequality (5.5) gives

$$
\begin{aligned}
& B\left(\mathbf{u}^{\mathcal{T}}, p^{\mathfrak{D}} ; \mathbf{v}^{\mathcal{T}}, 0\right) \geq-\overline{\mathrm{C}}_{\eta} C_{4} C_{8} C\left\|\nabla_{\mathfrak{Q}}^{\mathcal{N}} \mathbf{u}^{\mathcal{T}}\right\|_{2}\left\|p^{\mathfrak{Q}}\right\|_{2}+\left\|p^{\mathfrak{Q}}\right\|_{2}^{2} \\
& -\sum_{\mathcal{D} \in \mathfrak{D}} \int_{\mathcal{D}} p^{\mathcal{D}}\left(\operatorname{div}^{\mathcal{D}}\left(\mathbf{v}^{\mathcal{T}}\right)-\operatorname{div}(\mathbf{v}(z))\right) \mathrm{d} z-\sum_{\mathcal{D} \in \mathfrak{D}} \sum_{\mathcal{Q} \in \mathfrak{Q}_{\mathcal{D}}} \int_{\mathcal{Q}}\left(p^{\mathcal{D}}-p^{\mathcal{Q}}\right) \operatorname{div}(\mathbf{v}(z)) \mathrm{d} z .
\end{aligned}
$$


- Thanks to Lemma 4.3 and to estimate (5.4), we obtain

$$
\sum_{\mathcal{D} \in \mathfrak{D}} \int_{\mathcal{D}} p^{\mathcal{D}}\left(\operatorname{div}^{\mathcal{D}}\left(\mathbf{v}^{\mathcal{T}}\right)-\operatorname{div}(\mathbf{v}(z))\right) \mathrm{d} z \leq C C_{9}\left|p^{\mathfrak{D}}\right|_{h}\left\|p^{\mathfrak{Q}}\right\|_{2} .
$$

- Thanks to Lemma 2.3, we have $p^{\mathcal{D}}-p^{\mathcal{Q}}=-\alpha_{\mathfrak{s}, \mathcal{D}}\left(\mathrm{D}^{\mathcal{D}} \mathbf{u}^{\mathcal{T}}\right)$, with $\mathfrak{s}=\partial \mathcal{D} \cap \partial \mathcal{Q}$. Cauchy-Schwarz implies

$$
\sum_{\mathcal{D} \in \mathfrak{D}} \sum_{\mathcal{Q} \in \mathfrak{Q}_{\mathcal{D}}} \int_{\mathcal{Q}}\left(p^{\mathcal{D}}-p^{\mathcal{Q}}\right) \operatorname{div}(\mathbf{v}(z)) \mathrm{d} z \leq\|\operatorname{div}(\mathbf{v})\|_{2}\left(\sum_{\mathcal{D} \in \mathcal{D}} \sum_{\mathfrak{s} \in \mathcal{E}_{\mathcal{D}}} m_{\mathcal{D}}\left|\alpha_{\mathfrak{s}, \mathcal{D}}\left(D^{\mathcal{D}} \mathbf{u}^{\mathcal{T}}\right)\right|^{2}\right)^{\frac{1}{2}} .
$$

Thanks to $\operatorname{div}(\mathbf{v})=-p^{\mathfrak{Q}}$, Lemma 2.3 implies that

$$
\sum_{\mathcal{D} \in \mathfrak{D}} \sum_{\mathcal{Q} \in \mathfrak{Q}_{\mathcal{D}}} \int_{\mathcal{Q}}\left(p^{\mathcal{D}}-p^{\mathcal{Q}}\right) \operatorname{div}(\mathbf{v}(z)) \mathrm{d} z \leq \sqrt{C_{2}}\left\|p^{\mathfrak{Q}}\right\|_{2}\left\|\mathrm{D}^{\mathcal{D}} \mathbf{u}^{\mathcal{T}}\right\|_{2}
$$

Lemma 4.1 and Proposition 4.1 give

$$
\sum_{\mathcal{D} \in \mathfrak{D}} \sum_{\mathcal{Q} \in \mathfrak{Q}_{\mathcal{D}}} \int_{\mathcal{Q}}\left(p^{\mathcal{D}}-p^{\mathcal{Q}}\right) \operatorname{div}(\mathbf{v}(z)) \mathrm{d} z \leq \sqrt{C_{2}}\left\|p^{\mathfrak{Q}}\right\|_{2}\left\|\nabla_{\mathfrak{Q}}^{\mathcal{N}} \mathbf{u}^{\mathcal{T}}\right\|_{2}
$$

We then deduce from (5.6) that

$$
B\left(\mathbf{u}^{\mathcal{T}}, p^{\mathfrak{D}} ; \mathbf{v}^{\mathcal{T}}, 0\right) \geq\left\|p^{\mathfrak{Q}}\right\|_{2}^{2}-\overline{\mathrm{C}}_{\eta} C_{4} C_{8} C\left\|p^{\mathfrak{Q}}\right\|_{2}\left\|\nabla_{\mathfrak{Q}}^{\mathcal{N}} \mathbf{u}^{\mathcal{T}}\right\|_{2}-\left(\sqrt{C_{2}}+C C_{9}\right)\left|p^{\mathfrak{D}}\right|_{h}\left\|p^{\mathfrak{Q}}\right\|_{2}
$$

Using Young's inequality, we obtain the existence of three constants $\widetilde{C}_{1}, \widetilde{C}_{2}, \widetilde{C}_{3}>0$, depending only on $\Omega, \overline{\mathrm{C}}_{\eta}$ and $\operatorname{reg}(\mathcal{T})$, such that

$$
B\left(\mathbf{u}^{\mathcal{T}}, p^{\mathfrak{D}} ; \mathbf{v}^{\mathcal{T}}, 0\right) \geq \widetilde{C}_{1}\left\|p^{\mathfrak{Q}}\right\|_{2}^{2}-\widetilde{C}_{2}\left\|\nabla_{\mathfrak{Q}}^{\mathcal{N}} \mathbf{u}^{\mathcal{T}}\right\|_{2}^{2}-\widetilde{C}_{3}\left|p^{\mathfrak{D}}\right|_{h}^{2}
$$

Step 3. By bilinearity of $B,(5.3)$ and (5.7) give for each positive number $\xi>0$ :

$$
\begin{aligned}
& B\left(\mathbf{u}^{\mathcal{T}}, p^{\mathfrak{D}} ; \mathbf{u}^{\mathcal{T}}+\xi \mathbf{v}^{\mathcal{T}}, p^{\mathfrak{D}}\right) \\
& \quad \geq\left(\frac{1}{C_{5}^{2}}\left(2 \underline{\mathrm{C}}_{\eta}-C_{3} \frac{\lambda}{2}\right)-\xi \widetilde{C}_{2}\right)\left\|\nabla_{\mathfrak{Q}}^{\mathcal{N}} \mathbf{u}^{\mathcal{T}}\right\|_{2}^{2}+\xi \widetilde{C}_{1}\left\|p^{\mathfrak{Q}}\right\|_{2}^{2}+\left(\frac{\lambda}{2}-\xi \widetilde{C}_{3}\right)\left|p^{\mathfrak{D}}\right|_{h}^{2} .
\end{aligned}
$$

Choosing a value of $\xi>0$ small enough, this inequality yields an estimate of the form (5.2). As the relation (5.1) is clearly satisfied by the pair $\widetilde{\mathbf{u}}^{\mathcal{T}}=\mathbf{u}^{\mathcal{T}}+\xi \mathbf{v}^{\mathcal{T}}$ and $\widetilde{p}^{\mathfrak{D}}=p^{\mathfrak{D}}$, (since (5.5) and Theorem 4.2), this concludes the proof.

A consequence of this stability inequality is the well-posedness of the scheme (3.1).

THEOREM 5.2. Assume that $\eta$ satisfies (1.2). For any DDFV mesh $\mathcal{T}$, the finite volume scheme (3.1) with $0<\lambda<\frac{4 \underline{\mathrm{C}}_{\eta}}{C_{3}}$ admits a unique solution $\left(\mathbf{u}^{\mathcal{T}}, p^{\mathfrak{D}}\right) \in\left(\mathbb{R}^{2}\right)^{\mathcal{T}} \times \mathbb{R}^{\mathfrak{D}}$. Proof. Let us consider the homogeneous discrete problem given by setting $\mathbf{f}^{\mathcal{T}}$, the right-hand side of (3.1), to zero. Thanks to Theorem 5.1, there exists $\widetilde{\mathbf{u}}^{\mathcal{T}} \in \mathbb{E}_{0}, \widetilde{p}^{\mathfrak{D}} \in \mathbb{R}^{\mathfrak{D}}$, such that

$$
\left\|\nabla_{\mathfrak{Q}}^{\mathcal{N}} \mathbf{u}^{\mathcal{T}}\right\|_{2}^{2}+\left\|p^{\mathfrak{Q}}\right\|_{2}^{2} \leq C_{13} B\left(\mathbf{u}^{\mathcal{T}}, p^{\mathfrak{D}} ; \widetilde{\mathbf{u}}^{\mathcal{T}}, \widetilde{p}^{\mathfrak{D}}\right)
$$

Definition 5.1 of $B$ implies that $B\left(\mathbf{u}^{\mathcal{T}}, p^{\mathfrak{D}} ; \widetilde{\mathbf{u}}^{\mathcal{T}}, \widetilde{p}^{\mathfrak{D}}\right)=0$. It follows that $\nabla_{\mathfrak{Q}}^{\mathcal{N}} \mathbf{u}^{\mathcal{T}}=0$ and $p^{\mathfrak{Q}}=0$, with $\left(\delta^{\mathcal{D}}, p^{\mathfrak{Q}}\right)$ the solution of $(2.15)$ with $\mathrm{D}^{\mathfrak{D}} \mathbf{u}^{\mathcal{T}}$ and $p^{\mathfrak{D}}$. We deduce that $p^{\mathfrak{D}}=0$. The former identity implies that the degrees of freedom of the velocity $\mathbf{u}^{\mathcal{T}}$ are constant, since $\mathbf{u}^{\mathcal{T}} \in \mathbb{E}_{0}$, we conclude that $\mathbf{u}^{\mathcal{T}}=0$. 
6. Error estimates. In the following, we assume that the viscosity $\eta$ satisfies (1.2) and (2.1). In order to study the rates of convergence of our approximate solution, we need to make some assumptions on the regularity of a solution $(\mathbf{u}, p)$. In the following, we assume that $(\mathbf{u}, p)$ the solution of the problem $(1.1)$ lies in $\left(H^{2}(\mathfrak{Q})\right)^{2} \times H^{1}(\mathfrak{Q})$, that is:

$$
\begin{aligned}
\left(H^{2}(\mathfrak{Q})\right)^{2} & =\left\{\mathbf{u} \in\left(H^{1}(\Omega)\right)^{2}, \quad \mathbf{u}_{\left.\right|_{\mathcal{Q}}} \in\left(H^{2}(\mathcal{Q})\right)^{2}, \text { for all } \mathcal{Q} \in \mathfrak{Q}\right\}, \text { for the velocity, } \\
H^{1}(\mathfrak{Q}) & =\left\{p \in L^{2}(\Omega), \quad p_{\left.\right|_{\mathcal{Q}}} \in H^{1}(\mathcal{Q}), \text { for all } \mathcal{Q} \in \mathfrak{Q}\right\} \text { for the pressure, }
\end{aligned}
$$

with the corresponding norms

$$
\begin{aligned}
& \|\mathbf{u}\|_{\left(H^{2}(\mathfrak{Q})\right)^{2}}^{2}=\|\mathbf{u}\|_{H^{1}(\Omega)}^{2}+\sum_{\mathcal{Q} \in \mathfrak{Q}}\left\|\nabla^{2} \mathbf{u}\right\|_{L^{2}(\mathcal{Q})}^{2}, \quad \forall \mathbf{u} \in\left(H^{2}(\mathfrak{Q})\right)^{2}, \\
& \|p\|_{H^{1}(\mathfrak{Q})}^{2}=\|p\|_{L^{2}(\Omega)}^{2}+\sum_{\mathcal{Q} \in \mathfrak{Q}}\|\nabla p\|_{L^{2}(\mathcal{Q})}^{2}, \quad \forall p \in H^{1}(\mathfrak{Q}) .
\end{aligned}
$$

6.1. Definitions. We define projections of functions defined on $\Omega$ over the primal and dual meshes $\mathcal{T}$. We call the center-value projection for any continuous function $\mathbf{u}$ on $\bar{\Omega}$ :

$$
\mathbb{P}_{\boldsymbol{c}}^{\mathcal{T}} \mathbf{u}=\left(\left(\mathbf{u}\left(x_{\mathcal{K}}\right)\right)_{\mathcal{K} \in(\mathfrak{M} \cup \partial \mathfrak{M})},\left(\mathbf{u}\left(x_{\mathcal{K}^{*}}\right)\right)_{\mathcal{K}^{*} \in\left(\mathfrak{M}^{*} \cup \partial \mathfrak{M}^{*}\right)}\right), \quad \forall \mathbf{v} \in\left(H^{2}(\mathfrak{Q})\right)^{2}
$$

We also define a mean-value projection over the diamond mesh $\mathfrak{D}$ and over the quarter diamond mesh $\mathcal{Q}$ for any integrable functions $q$ on $\Omega$ :

$$
\mathbb{P}_{m}^{\mathfrak{D}} q=\left(\left(\frac{1}{m_{\mathcal{D}}} \int_{\mathcal{D}} q(x) \mathrm{d} x\right)_{\mathcal{D} \in \mathfrak{D}}\right), \quad \mathbb{P}_{m}^{\mathfrak{Q}} q=\left(\left(\frac{1}{m_{\mathcal{Q}}} \int_{\mathcal{Q}} q(x) \mathrm{d} x\right)_{\mathcal{Q} \in \mathfrak{Q}}\right) .
$$

The following proposition is a consequence of Theorem 2.1 .

Proposition 6.1. Let $(\mathbf{u}, p)$ the solution of the Stokes problem (1.1). There exists $\left(\delta_{\mathrm{ex}}^{\mathcal{D}}, p_{\mathrm{ex}}^{\mathfrak{Q}_{\mathcal{D}}}\right) \in \mathcal{M}_{n_{\mathcal{D}}, 2}(\mathbb{R}) \times \mathbb{R}^{n_{\mathcal{D}}}$ satisfying

$$
\left\{\begin{array}{l}
\sum_{\mathcal{Q} \in \mathfrak{Q}_{\mathcal{D}}} m_{\mathcal{Q}} \varphi_{\mathcal{Q}}\left(\mathrm{D}^{\mathcal{D}} \mathbb{P}_{c}^{\mathcal{T}} \mathbf{u}, \delta_{\mathrm{ex}}^{\mathcal{D}}, p_{\mathrm{ex}}^{\mathcal{Q}}\right) B_{\mathcal{Q}}=0, \quad \operatorname{Tr}\left({ }^{t} \delta_{\mathrm{ex}}^{\mathcal{D} t} B_{\mathcal{Q}}\right)=0, \forall \mathcal{Q} \in \mathfrak{Q}_{\mathcal{D}}, \\
\sum_{\mathcal{Q} \in \mathfrak{Q}_{\mathcal{D}}} m_{\mathcal{Q}} p_{\mathrm{ex}}^{\mathcal{Q}}=m_{\mathcal{D}} \mathbb{P}_{m}^{\mathcal{D}} p, \quad\left(\delta_{\mathrm{ex}}^{\mathcal{D}}: \delta_{0}\right)=0 .
\end{array}\right.
$$

Thanks to Proposition 6.1, in the following, we note

$$
\widetilde{p}_{\mathrm{ex}}^{\mathcal{Q}}=\mathbb{P}_{m}^{\mathcal{Q}} p-p_{\mathrm{ex}}^{\mathcal{Q}}, \forall \mathcal{Q} \in \mathfrak{Q} \text { and we have } \sum_{\mathcal{Q} \in \mathfrak{Q}_{\mathcal{D}}} m_{\mathcal{Q}} \widetilde{p}_{\mathrm{ex}}^{\mathcal{Q}}=0 .
$$

As usual for the error analysis of the finite volume methods, the consistency error which has to be studied is the error on the numerical fluxes across each of the primal and dual edges of the mesh. We first give the precise definition of these terms, then we state the various estimates needed to prove the error estimates.

DEFINITION 6.1. For any $\mathcal{Q} \in \mathfrak{Q}$, we define the consistency errors in $\mathcal{Q}$ by

$$
R_{\mathcal{Q}}^{\mathbf{u}}(z)=\eta_{\mid \overline{\mathcal{Q}}}(z) \mathrm{Du}_{\mid \overline{\mathcal{Q}}}(z)-\eta_{\mathcal{Q}} \mathrm{D}_{\mathcal{Q}}^{\mathcal{N}} \mathbb{P}_{\boldsymbol{c}}^{\mathcal{T}} \mathbf{u}, \quad R_{\mathcal{Q}}^{p}(z)=\mathbb{P}_{m}^{\mathcal{Q}} p-p_{\mid \overline{\mathcal{Q}}}(z), \quad \forall z \in \mathcal{D} .
$$

We introduce the following consistency errors on the numerical fluxes, for all $\mathfrak{s}=\mathcal{Q} \mid \mathcal{Q}^{\prime} \in \mathcal{E}_{\mathcal{Q}}$ :

$$
\mathbf{R}_{\mathfrak{s}, \mathcal{Q}}^{i}=-\mathbf{R}_{\mathfrak{s}, \mathcal{Q}^{\prime}}^{i}=\frac{1}{m_{\mathfrak{s}}} \int_{\mathfrak{s}} R_{\mathcal{Q}}^{i}(z) \overrightarrow{\mathbf{n}}_{\mathfrak{s} \mathcal{Q}} \mathrm{d} z, \quad \text { for } i=\mathbf{u}, p .
$$

We note the $L^{2}(\Omega)$-norm of the consistency error as follows:

$$
\left\|\mathbf{R}_{\mathfrak{s}, \mathfrak{Q}}^{i}\right\|_{2}^{2}=\sum_{\mathcal{Q} \in \mathfrak{Q}} \sum_{\mathfrak{s} \in \mathcal{E}_{\mathcal{Q}}} m_{\mathcal{Q}}\left|\mathbf{R}_{\mathfrak{s}, \mathcal{Q}}^{i}\right|^{2}, \quad \text { for } i=\mathbf{u}, p .
$$


Thanks to (6.3), we define for all $\mathfrak{s}=\mathcal{Q} \mid \mathcal{Q}^{\prime} \in \mathcal{E}_{\mathcal{Q}}$ :

$$
\mathbf{R}_{\mathfrak{s}, \mathcal{Q}}=2 \mathbf{R}_{\mathfrak{s}, \mathcal{Q}}^{\mathbf{u}}+\mathbf{R}_{\mathfrak{s}, \mathcal{Q}}^{p}-\widetilde{p}_{\mathrm{ex}}^{\mathcal{Q}} \overrightarrow{\mathbf{n}}_{\mathfrak{s} \mathcal{Q}}
$$

6.2. Statement of the result and sketch of proof. The main result of the Section 6 is the following.

THEOREM 6.1. Assume that $\eta$ satisfies (1.2) and (2.1) and $0<\lambda<\frac{4 \underline{\mathrm{C}}_{\eta}}{C_{3}}$. We assume that the solution $(\mathbf{u}, p)$ of the Stokes problem $(1.1)$ belongs to $\left(H^{2}(\mathfrak{Q})\right)^{2} \times H^{1}(\mathfrak{Q})$. Let $\left(\mathbf{u}^{\mathcal{T}}, p^{\mathfrak{D}}\right) \in\left(\mathbb{R}^{2}\right)^{\mathcal{T}} \times \mathbb{R}^{\mathfrak{D}}$ be the solution of the scheme $(3.1)$. There exists a constant $C_{14}>0$ depending only on $\operatorname{reg}(\mathcal{T}), \lambda, \sin \left(\epsilon_{0}\right), \mathrm{C}_{\eta}, \underline{\mathrm{C}}_{\eta}, \overline{\mathrm{C}}_{\eta},\|\mathbf{u}\|_{\left(H^{2}(\mathfrak{Q})\right)^{2}}$ and $\|p\|_{H^{1}(\mathfrak{Q})}$, such that:

$$
\left\|\mathbf{u}-\mathbf{u}^{\mathcal{T}}\right\|_{2}+\left\|\mathrm{Du}-\mathrm{D}_{\mathfrak{Q}}^{\mathcal{N}} \mathbf{u}^{\mathcal{T}}\right\|_{2} \leq C_{14} \operatorname{size}(\mathcal{T}) \quad \text { and } \quad\left\|p-p^{\mathfrak{Q}}\right\|_{2} \leq C_{14} \operatorname{size}(\mathcal{T}) .
$$

with $\left(\delta^{\mathfrak{D}}, p^{\mathfrak{Q}}\right)$ the solution of (2.15) for $\mathrm{D}^{\mathfrak{D}} \mathbf{u}^{\mathcal{T}}$ and $p^{\mathfrak{D}}$.

Step 1. Let $\mathbf{e}^{\mathcal{T}}=\mathbb{P}_{c}^{\mathcal{T}} \mathbf{u}-\mathbf{u}^{\mathcal{T}} \in \mathbb{E}_{0}$ denote the approximation error for the velocity field and $e^{\mathfrak{D}}=\mathbb{P}_{m}^{\mathfrak{D}} p-p^{\mathfrak{D}} \in \mathbb{R}^{\mathfrak{D}}$ the approximation error for the pressure field. Thanks to (3.1) and (1.1), we have $\forall \mathcal{K} \in \mathfrak{M}$

$$
\operatorname{div}^{\mathcal{K}}\left(-2 \mathrm{D}_{\mathfrak{D}}^{\eta, \mathcal{N}} \mathbf{u}^{\mathcal{T}}+p^{\mathfrak{D}} \mathrm{Id}\right)=\mathbf{f}_{\mathcal{K}}=-\frac{1}{m_{\mathcal{K}}} \int_{\mathcal{K}} \operatorname{div}(2 \eta(x) \operatorname{Du}(x)) \mathrm{d} x+\frac{1}{m_{\mathcal{K}}} \int_{\mathcal{K}} \nabla p(x) \mathrm{d} x .
$$

Therefore, Definition 2.2 of $\operatorname{div}^{\mathcal{K}}$ and the continuous Stokes formula imply that

$$
\begin{aligned}
& m_{\mathcal{K}} \operatorname{div}^{\mathcal{K}}\left(-2 \mathrm{D}_{\mathfrak{D}}^{\eta, \mathcal{N}} \mathbf{e}^{\mathcal{T}}+e^{\mathfrak{D}} I d\right)=\sum_{\mathcal{Q} \subset \mathcal{K}} \sum_{\substack{\mathfrak{s} \in \mathcal{E}_{\mathcal{Q}} \\
\mathfrak{s} \subset \partial \mathcal{K}}} \int_{\mathfrak{s}} 2 \eta(z) \operatorname{Du}(z) \overrightarrow{\mathbf{n}}_{\mathfrak{s} \mathcal{Q}} \mathrm{d} z \\
& -\sum_{\mathcal{D} \in \mathfrak{D}_{\mathcal{K}}} m_{\sigma}\left(2 \mathrm{D}_{\mathcal{D}}^{\eta, \mathcal{N}} \mathbb{P}_{\boldsymbol{c}}^{\mathcal{T}} \mathbf{u}\right) \overrightarrow{\mathbf{n}}_{\sigma \mathcal{K}}+\sum_{\mathcal{D} \in \mathfrak{D}_{\mathcal{K}}} m_{\sigma} \mathbb{P}_{m}^{\mathcal{D}} p \overrightarrow{\mathbf{n}}_{\sigma \mathcal{K}}-\sum_{\mathcal{Q} \subset \mathcal{K}} \sum_{\mathfrak{s} \in \mathcal{E}_{\mathcal{Q}}} \int_{\mathfrak{s} \subset \partial \mathcal{K}} p(z) \overrightarrow{\mathbf{n}}_{\mathfrak{s} \mathcal{Q}} \mathrm{d} z
\end{aligned}
$$

Using Definition 2.8 of $\varphi_{\mathcal{Q}}$ and Proposition 6.1, we deduce for any $\mathcal{D} \in \mathfrak{D}_{\mathcal{K}}$,

$$
\frac{m_{\sigma}}{m_{\mathcal{D}}} \sum_{\mathcal{Q} \in \mathfrak{Q}_{\mathcal{D}}} m_{\mathcal{Q}} \varphi_{\mathcal{Q}}\left(\mathrm{D}^{\mathcal{D}} \mathbb{P}_{\boldsymbol{c}}^{\mathcal{T}} \mathbf{u}, \delta_{\mathrm{ex}}^{\mathcal{D}}, p_{\mathrm{ex}}^{\mathfrak{Q}_{\mathcal{D}}}\right) \overrightarrow{\mathbf{n}}_{\sigma \mathcal{K}}=m_{\sigma_{\mathcal{K}^{*}}} \varphi_{\mathcal{Q}_{\mathcal{K}, \mathcal{K}^{*}}} \overrightarrow{\mathbf{n}}_{\sigma \mathcal{K}}+m_{\sigma_{\mathcal{L}^{*}}} \varphi_{\mathcal{Q}_{\mathcal{K}, \mathcal{L}^{*}}} \overrightarrow{\mathbf{n}}_{\sigma \mathcal{K}}
$$

Thanks to Definition 6.1 of the consistency error and (6.3), we deduce

$$
m_{\mathcal{K}} \operatorname{div}^{\mathcal{K}}\left(-2 \mathrm{D}_{\mathfrak{D}}^{\eta, \mathcal{N}} \mathbf{e}^{\mathcal{T}}+e^{\mathfrak{D}} I d\right)=\sum_{\substack{\mathcal{Q} \subset \mathcal{K} \\ s \in \mathcal{E}_{\mathcal{Q}} \\ \mathfrak{s} \subset \partial \mathcal{K}}} m_{\mathfrak{s}} \mathbf{R}_{\mathfrak{s}, \mathcal{Q}}
$$

We do similar computations for $\mathcal{K}^{*} \in \mathfrak{M}^{*}$. Finally, the couple $\left(\mathbf{e}^{\mathcal{T}}, e^{\mathfrak{D}}\right) \in \mathbb{E}_{0} \times \mathbb{R}^{\mathfrak{D}}$ satisfies:

$$
\begin{cases}\operatorname{div}^{\mathfrak{M}}\left(-2 \mathrm{D}_{\mathfrak{D}}^{\eta, \mathcal{N}} \mathbf{e}^{\mathcal{T}}+e^{\mathfrak{D}} \mathrm{Id}\right)=\mathbf{R}_{\mathfrak{M}}, & \operatorname{div}^{\mathfrak{M} *}\left(-2 \mathrm{D}_{\mathfrak{D}}^{\eta, \mathcal{N}} \mathbf{e}^{\mathcal{T}}+e^{\mathfrak{D}} \mathrm{Id}\right)=\mathbf{R}_{\mathfrak{M}^{*}}, \\ \operatorname{div}^{\mathfrak{D}}\left(\mathbf{e}^{\mathcal{T}}\right)-\lambda h_{\mathfrak{D}}^{2} \underline{\Delta}^{\mathfrak{D}} e^{\mathfrak{Q}}=R_{\mathfrak{D}}, & \sum_{\mathcal{D} \in \mathfrak{D}} m_{\mathcal{D}} e^{\mathcal{D}}=0,\end{cases}
$$

where $\mathbf{R}_{\mathfrak{M}}=\left(\mathbf{R}_{\mathcal{K}}\right)_{\mathcal{K} \in \mathfrak{M}}, \mathbf{R}_{\mathfrak{M}^{*}}=\left(\mathbf{R}_{\mathcal{K}^{*}}\right)_{\mathcal{K}^{*} \in \mathfrak{M}^{*}}$ and $R_{\mathfrak{D}}=\left(R_{\mathcal{D}}\right)_{\mathcal{D} \in \mathfrak{D}}$ with:

$$
\begin{aligned}
& \mathbf{R}_{\mathcal{K}}=\frac{1}{m_{\mathcal{K}}} \sum_{\mathcal{Q} \subset \mathcal{K}} \sum_{\substack{\mathfrak{s} \in \mathcal{E}_{\mathcal{Q}} \\
\mathfrak{s} \subset \partial \mathcal{K}}} m_{\mathfrak{s}} \mathbf{R}_{\mathfrak{s}, \mathcal{Q}}, \forall \mathcal{K} \in \mathfrak{M}, \quad \mathbf{R}_{\mathcal{K}^{*}}=\frac{1}{m_{\mathcal{K}^{*}}} \sum_{\mathcal{Q} \subset \mathcal{K}^{*}} \sum_{\substack{\mathfrak{s} \in \mathcal{E}_{\mathcal{Q}} \\
\mathfrak{s} \subset \partial \mathcal{K}^{*}}} m_{\mathfrak{s}} \mathbf{R}_{\mathfrak{s}, \mathcal{Q}}, \forall \mathcal{K}^{*} \in \mathfrak{M}^{*}, \\
& R_{\mathcal{D}}=\operatorname{div}^{\mathcal{D}}\left(\mathbb{P}_{c}^{\mathcal{T}} \mathbf{u}\right)-\lambda h_{\mathcal{D}}^{2} \underline{\Delta}^{\mathcal{D}} p_{\mathrm{ex}}^{\mathfrak{Q}}, \forall \mathcal{D} \in \mathfrak{D}, \quad e^{\mathcal{Q}}=p_{\mathrm{ex}}^{\mathcal{Q}}-p^{\mathcal{Q}}, \forall \mathcal{Q} \in \mathfrak{Q} .
\end{aligned}
$$


Theorem 5.1 implies that there exists $\widetilde{\mathbf{e}}^{\mathcal{T}} \in \mathbb{E}_{0}, \widetilde{e}^{\mathfrak{D}} \in \mathbb{R}^{\mathfrak{D}}$ such that :

$$
\left\|\nabla_{\mathfrak{Q}}^{\mathcal{N}} \widetilde{\mathbf{e}}^{\mathcal{T}}\right\|_{2}+\left\|\widetilde{e}^{\mathfrak{Q}}\right\|_{2} \leq C_{12}\left(\left\|\nabla_{\mathfrak{Q}}^{\mathcal{N}} \mathbf{e}^{\mathcal{T}}\right\|_{2}+\left\|e^{\mathfrak{Q}}\right\|_{2}\right)
$$

and

$$
\left\|\nabla_{\mathfrak{Q}}^{\mathcal{N}} \mathbf{e}^{\mathcal{T}}\right\|_{2}^{2}+\left\|e^{\mathfrak{Q}}\right\|_{2}^{2} \leq C_{13} B\left(\mathbf{e}^{\mathcal{T}}, e^{\mathfrak{D}} ; \widetilde{\mathbf{e}}^{\mathcal{T}}, \widetilde{e}^{\mathfrak{D}}\right) .
$$

Thanks to Definition 5.1 of $B$ and to (6.5), we have $B\left(\mathbf{e}^{\mathcal{T}}, e^{\mathfrak{D}} ; \widetilde{\mathbf{e}}^{\mathcal{T}}, \widetilde{e}^{\mathfrak{D}}\right)=I+T$, with $I:=\llbracket \operatorname{div}^{\mathcal{T}}\left(-2 \mathrm{D}_{\mathfrak{D}}^{\eta, \mathcal{N}} \mathbf{e}^{\mathcal{T}}+e^{\mathfrak{D}} \mathrm{Id}\right), \widetilde{\mathbf{e}}^{\mathcal{T}} \rrbracket$ and $T:=\left(\operatorname{div}^{\mathfrak{D}}\left(\mathbf{e}^{\mathcal{T}}\right)-\lambda h_{\mathfrak{D}}^{2} \underline{\Delta}^{\mathfrak{D}} e^{\mathfrak{Q}}, \widetilde{e}^{\mathfrak{D}}\right)_{\mathfrak{D}}$. Using the fact that $\widetilde{\mathbf{e}}_{\mathcal{K}^{*}}=0$ for any $\mathcal{K}^{*} \in \partial \mathfrak{M}^{*}$ and the definition of $I$, we have

$$
I=\sum_{\mathcal{K} \in \mathfrak{M}} \sum_{\mathcal{Q} \subset \mathcal{K}} \sum_{\substack{\mathfrak{s} \in \mathcal{E}_{\mathcal{Q}} \\ \mathfrak{s} \subset \partial \mathcal{K}}} m_{\mathfrak{s}}\left(\mathbf{R}_{\mathfrak{s}, \mathcal{Q}}, \widetilde{\mathbf{e}}_{\mathcal{K}}\right)+\sum_{\mathcal{K}^{*} \in \mathfrak{M}^{*} \cup \partial \mathfrak{M}^{*}} \sum_{\mathcal{Q} \subset \mathcal{K}^{*}} \sum_{\substack{\mathfrak{s} \in \mathcal{E}_{\mathcal{Q}} \\ \mathfrak{s} \subset \partial \mathcal{K}^{*}}} m_{\mathfrak{s}}\left(\mathbf{R}_{\mathfrak{s}, \mathcal{Q}}, \widetilde{\mathbf{e}}_{\mathcal{K}^{*}}\right) .
$$

Using the fact that $\mathbf{R}_{\sigma_{\mathcal{K}}, \mathcal{Q}_{\mathcal{K}, \mathcal{K}^{*}}}=-\mathbf{R}_{\sigma_{\mathcal{K}}, \mathcal{Q}_{\mathcal{K}, \mathcal{L}^{*}}}$, we have

$$
\begin{aligned}
& m_{\sigma_{\mathcal{K}}}\left(\left(\mathbf{R}_{\sigma_{\mathcal{K}}, \mathcal{Q}_{\mathcal{K}, \mathcal{K}^{*}}}, \widetilde{\mathbf{e}}_{\mathcal{K}^{*}}\right)+\left(\mathbf{R}_{\sigma_{\mathcal{K}}, \mathcal{Q}_{\mathcal{K}, \mathcal{L}^{*}}}, \widetilde{\mathbf{e}}_{\mathcal{L}^{*}}\right)\right) \\
& =-\frac{2}{\sin \left(\alpha_{\mathcal{K}}\right)}\left(m_{\mathcal{Q}_{\mathcal{K}, \mathcal{K}^{*}}} \mathbf{R}_{\sigma_{\mathcal{K}}, \mathcal{Q}_{\mathcal{K}, \mathcal{K}^{*}}}+m_{\mathcal{Q}_{\mathcal{K}, \mathcal{L}^{*}}} \mathbf{R}_{\sigma_{\mathcal{K}}, \mathcal{Q}_{\mathcal{K}, \mathcal{L}^{*}}}\right) \cdot\left(\nabla^{\mathcal{D}} \widetilde{\mathbf{e}}^{\mathcal{T}} \vec{\tau}_{\mathcal{K}^{*}, \mathcal{L}^{*}}\right) .
\end{aligned}
$$

Reorganizing the sum over all the diamond cells $\mathcal{D} \in \mathfrak{D}$, we deduce

$$
I \leq \frac{4}{\sin \left(\alpha_{\mathcal{T}}\right)} \sum_{\mathcal{D} \in \mathfrak{D}} \sum_{\mathcal{Q} \in \mathfrak{Q}_{\mathcal{D}}} \sum_{\mathfrak{s} \in \mathcal{E}_{\mathcal{Q}}} m_{\mathcal{Q}}\left|\mathbf{R}_{\mathfrak{s}, \mathcal{Q}}\right|\left\|\nabla^{\mathcal{D}} \widetilde{\mathbf{e}}^{\mathcal{T}}\right\|_{\mathcal{F}}
$$

Thanks to the Cauchy-Schwarz inequality and to Lemma 4.2, we obtain

$$
I \leq \frac{4}{\sin \left(\alpha_{\mathcal{T}}\right)}\left\|\mathbf{R}_{\mathfrak{s}, \mathfrak{Q}}\right\|_{2}\left\|\nabla_{\mathfrak{Q}}^{\mathcal{N}} \widetilde{\mathbf{e}}^{\mathcal{T}}\right\|_{2}
$$

We note $T_{1}:=-\lambda\left(h_{\mathfrak{D}}^{2} \underline{\Delta}^{\mathfrak{D}} p_{\text {ex }}^{\mathfrak{Q}}, \widetilde{e}^{\mathfrak{D}}\right)_{\mathfrak{D}}$. Reordering the summation over $\mathfrak{s} \in \mathfrak{S}$, we have

$$
T_{1}=\lambda \sum_{\mathfrak{s}=\mathcal{Q}\left|\mathcal{Q}^{\prime}=\mathcal{D}\right| \mathcal{D}^{\prime} \in \mathfrak{S}}\left(h_{\mathcal{D}}^{2}+h_{\mathcal{D}^{\prime}}^{2}\right)\left(p_{\mathrm{ex}}^{\mathcal{Q}^{\prime}}-p_{\mathrm{ex}}^{\mathcal{Q}}\right)\left(\widetilde{e}^{\mathcal{D}^{\prime}}-\widetilde{e}^{\mathcal{D}}\right) .
$$

The Cauchy-Schwarz inequality and Lemma 4.4 give

$$
\left|T_{1}\right| \leq C_{10} 2 \operatorname{size}(\mathcal{T}) \lambda\left\|\tilde{e}^{\mathfrak{D}}\right\|_{2}\left(\sum_{\mathfrak{s}=\mathcal{Q}\left|\mathcal{Q}^{\prime}=\mathcal{D}\right| \mathcal{D}^{\prime} \in \mathfrak{S}}\left(p_{\mathrm{ex}}^{\mathcal{Q}^{\prime}}-p_{\mathrm{ex}}^{\mathcal{Q}}\right)^{2}\right)^{\frac{1}{2}}
$$

For the term $T$, we have the following estimate:

$$
|T| \leq\left\|\widetilde{e}^{\mathfrak{D}}\right\|_{2}\left(2 \operatorname{size}(\mathcal{T}) \lambda C_{10}\left(\sum_{\mathfrak{s}=\mathcal{Q}\left|\mathcal{Q}^{\prime}=\mathcal{D}\right| \mathcal{D}^{\prime} \in \mathfrak{S}}\left(p_{\mathrm{ex}}^{\mathcal{Q}^{\prime}}-p_{\mathrm{ex}}^{\mathcal{Q}}\right)^{2}\right)^{\frac{1}{2}}+\left\|\operatorname{div}^{\mathfrak{D}} \mathbb{P}_{c}^{\mathcal{T}} \mathbf{u}\right\|_{2}\right)
$$

To sum up, using the fact that $\left\|\widetilde{e}^{\mathfrak{D}}\right\|_{2} \leq\left\|\widetilde{e}^{\mathfrak{Q}}\right\|_{2}$ and (6.6), (6.7) becomes

$$
\begin{aligned}
\left\|\nabla_{\mathfrak{Q}}^{\mathcal{N}} \mathbf{e}^{\mathcal{T}}\right\|_{2}^{2} & +\left\|e^{\mathfrak{Q}}\right\|_{2}^{2} \\
\leq & \widetilde{C}_{1}\left(\left\|\nabla_{\mathfrak{Q}}^{\mathcal{N}} \mathbf{e}^{\mathcal{T}}\right\|_{2}+\left\|e^{\mathfrak{Q}}\right\|_{2}\right)\left(\left\|\mathbf{R}_{\mathfrak{s}, \mathfrak{Q}}\right\|_{2}+\left\|\operatorname{div}^{\mathfrak{D}} \mathbb{P}_{\boldsymbol{c}}^{\mathcal{T}} \mathbf{u}\right\|_{2}\right) \\
& +\widetilde{C}_{2} \operatorname{size}(\mathcal{T})\left(\left\|\nabla_{\mathfrak{Q}}^{\mathcal{N}} \mathbf{e}^{\mathcal{T}}\right\|_{2}+\left\|e^{\mathfrak{Q}}\right\|_{2}\right)\left(\sum_{\mathfrak{s}=\mathcal{Q}\left|\mathcal{Q}^{\prime}=\mathcal{D}\right| \mathcal{D}^{\prime} \in \mathfrak{S}}\left(p_{\mathrm{ex}}^{\mathcal{Q}^{\prime}}-p_{\text {ex }}^{\mathcal{Q}}\right)^{2}\right)^{\frac{1}{2}}
\end{aligned}
$$

where $\widetilde{C}_{1}=C_{13} C_{12} \max \left(\frac{4}{\sin \left(\alpha_{\mathcal{T}}\right)}, 1\right)$ and $\widetilde{C}_{2}=2 C_{13} C_{12} \lambda C_{10}$. It remains to estimate the consistency errors. 
6.3. Consistency error for the pressure. LEMmA 6.1. There exists $C_{15}, C_{16}>0$, depending only on $\operatorname{reg}(\mathcal{T})$, such that for all $p \in H^{1}(\mathfrak{Q})$ and for all $\mathcal{D} \in \mathfrak{D}$

$$
\sum_{\mathcal{Q} \in \mathfrak{Q}_{\mathcal{D}}} m_{\mathcal{Q}} \sum_{\mathfrak{s} \in \mathcal{E}_{\mathcal{Q}}}\left|\mathbf{R}_{\mathfrak{s}, \mathcal{Q}}^{p}\right|^{2} \leq C_{15} h_{\mathcal{D}}^{2} \sum_{\mathcal{Q} \in \mathfrak{Q}_{\mathcal{D}}} \int_{\mathcal{Q}}|\nabla p(z)|^{2} \mathrm{~d} z
$$

and

$$
\left\|R_{\mathfrak{Q}}^{p}\right\|_{2}^{2} \leq C_{16} \operatorname{size}(\mathcal{T})^{2}\|p\|_{H^{1}(\mathfrak{Q})}^{2} .
$$

Proof. Definition 6.1 gives

$$
\sum_{\mathcal{Q} \in \mathfrak{Q}_{\mathcal{D}}} \sum_{\mathfrak{s} \in \mathcal{E}_{\mathcal{Q}}} m_{\mathcal{Q}}\left|\mathbf{R}_{\mathfrak{s}, \mathcal{Q}}^{p}\right|^{2} \leq h_{\mathcal{D}}^{2} \sum_{\mathcal{Q} \in \mathfrak{Q}_{\mathcal{D}}} \sum_{\mathfrak{s} \in \mathcal{E}_{\mathcal{Q}}}\left|\frac{1}{m_{\mathfrak{s}}} \int_{\mathfrak{s}}\left(\mathbb{P}_{m}^{\mathcal{Q}} p-p(z)\right) \mathrm{d} z\right|^{2}
$$

Thanks to Lemma 4.5 and $\frac{h_{\mathcal{Q}}^{3}}{m_{\mathfrak{s}} m_{\mathcal{Q}}} \leq \operatorname{reg}(\mathcal{T})^{3}$, we have

$$
\left|\frac{1}{m_{\mathfrak{s}}} \int_{\mathfrak{s}}\left(\mathbb{P}_{m}^{\mathcal{Q}} p-p(z)\right) \mathrm{d} z\right|^{2} \leq \frac{C_{11} h_{\mathcal{Q}}^{3}}{m_{\mathfrak{s}} m_{\mathcal{Q}}} \int_{\mathcal{Q}}|\nabla p(z)|^{2} \mathrm{~d} z \leq C_{11} \operatorname{reg}(\mathcal{T})^{3} \int_{\mathcal{Q}}|\nabla p(z)|^{2} \mathrm{~d} z,
$$

which concludes the first estimate. For the second estimate, we add and subtract $\frac{1}{m_{\mathfrak{s}}} \int_{\mathfrak{s}} p(x) \mathrm{d} x$ on $R_{\mathcal{Q}}^{p}(z)$ to get

$$
\int_{\mathcal{Q}}\left(p(z)-\mathbb{P}_{m}^{\mathcal{Q}} p\right)^{2} \mathrm{~d} z \leq 2 \int_{\mathcal{Q}}\left|\frac{1}{m_{\mathfrak{s}}} \int_{\mathfrak{s}}(p(z)-p(x)) \mathrm{d} x \mathrm{~d} z\right|^{2}+2 m_{\mathcal{Q}}\left|\frac{1}{m_{\mathfrak{s}}} \int_{\mathfrak{s}}\left(\mathbb{P}_{m}^{\mathcal{Q}} p-p(z)\right) \mathrm{d} z\right|^{2} .
$$

The Jensen inequality implies that

$$
\int_{\mathcal{Q}}\left(p(z)-\mathbb{P}_{m}^{\mathcal{Q}} p\right)^{2} \mathrm{~d} z \leq 4 m_{\mathcal{Q}} \frac{1}{m_{\mathcal{Q}} m_{\mathfrak{s}}} \int_{\mathcal{Q}} \int_{\mathfrak{s}}(p(z)-p(x))^{2} \mathrm{~d} x \mathrm{~d} z .
$$

Like in the estimate (6.9), we get the result with $C_{16}=4 C_{11} \operatorname{reg}(\mathcal{T})^{3}$.

\subsection{Consistency error for the velocity.}

6.4.1. Properties of the center-value projection operator. By using usual Taylor formulas inside each quarter diamond $\mathcal{Q}$ (see [5], for instance), we can easily show the main properties of the center-value projection for functions in $\left(H^{2}(\mathfrak{Q})\right)^{2}$.

Lemma 6.2. There exists $C_{17}, C_{18}>0$, depending only on $\operatorname{reg}(\mathcal{T})$, such that for any function $\mathbf{v}$ in $\left(H^{2}(\mathfrak{Q})\right)^{2}$, we have

$$
\left\|\mathbf{v}-\mathbb{P}_{\boldsymbol{c}}^{\mathcal{T}} \mathbf{v}\right\|_{2} \leq C_{17} \operatorname{size}(\mathcal{T})\|\mathbf{v}\|_{\left(H^{2}(\mathfrak{Q})\right)^{2}} \quad \text { and } \quad\left\|\nabla_{\mathfrak{Q}}^{\mathcal{N}} \mathbb{P}_{c}^{\mathcal{T}} \mathbf{v}\right\|_{2} \leq C_{18}\|\mathbf{v}\|_{\left(H^{2}(\mathfrak{Q})\right)^{2}} .
$$

6.4.2. Definitions. Definition 6.2. The consistency error $R_{\mathcal{Q}}^{\mathrm{u}}$ can be split into two different contributions $R_{Q}^{\mathbf{u}, \eta}$ and $R_{Q}^{\mathbf{u}, D u}$. They originate, resp., from the errors due to the approximation with respect to the space variable of the viscous stress tensor and to the approximation of the gradient: $R_{\mathcal{Q}}^{\mathbf{u}}(z)=R_{\mathcal{Q}}^{\mathbf{u}, \eta}(z)+R_{\mathcal{Q}}^{\mathbf{u}, \text { Du}}$, where

$$
\begin{aligned}
R_{\mathcal{Q}}^{\mathbf{u}, \eta}(z) & =\eta_{\mid \overline{\mathcal{Q}}}(z) \mathrm{D} \mathbf{u}_{\mid \overline{\mathcal{Q}}}(z)-\frac{1}{m_{\mathcal{Q}}} \int_{\mathcal{Q}} \eta(x) \mathrm{Du}(x) \mathrm{d} x, \\
R_{\mathcal{Q}}^{\mathbf{u}, \mathrm{D} \mathbf{u}} & =\frac{1}{m_{\mathcal{Q}}} \int_{\mathcal{Q}} \eta(x)\left(\mathrm{Du}(x)-\mathrm{D}_{\mathcal{Q}}^{\mathcal{N}} \mathbb{P}_{\boldsymbol{c}}^{\mathcal{T}} \mathbf{u}\right) \mathrm{d} x .
\end{aligned}
$$


We also introduce the quantity, for all $\mathfrak{s}=\mathcal{Q} \mid \mathcal{Q}^{\prime} \in \mathcal{E}_{\mathcal{Q}}$ :

$$
\mathbf{R}_{\mathfrak{s}, \mathcal{Q}}^{\mathbf{u}, \eta}=-\mathbf{R}_{\mathfrak{s}, \mathcal{Q}^{\prime}}^{\mathbf{u}, \eta}=\frac{1}{m_{\mathfrak{s}}} \int_{\mathfrak{s}} R_{\mathcal{Q}}^{\mathbf{u}, \eta}(z) \overrightarrow{\mathbf{n}}_{\mathfrak{s} \mathcal{Q}} \mathrm{d} z .
$$

DEFINITION 6.3. We define the projection $\mathbb{P}_{c}^{\mathfrak{Q}} \mathbf{u}$ of $\mathbf{u}$ on the set of quarter diamond cells as follows. For each quarter diamond cell $\mathcal{Q} \in \mathfrak{Q}$, the restriction of $\mathbb{P}_{c}^{\mathfrak{Q}} \mathbf{u}$ to the triangle $\mathcal{Q}$ is the unique affine function $\mathbb{P}_{c}^{\mathcal{Q}} \mathbf{u}$ which coincides with $\mathbf{u}$ at the middle point of the semiedges $\mathfrak{s} \in \mathcal{E}_{\mathcal{Q}}$ and whose value at the middle point of the third side of $\mathcal{Q}$ is the mean-value of the value $\mathbf{u}$ at the extremities of this side. Remark that this definition makes sense since $\mathbf{u}_{\mid \overline{\mathcal{Q}}} \in\left(H^{2}(\mathcal{Q})\right)^{2} \subset\left(\mathcal{C}^{0}(\overline{\mathcal{Q}})\right)^{2}$.

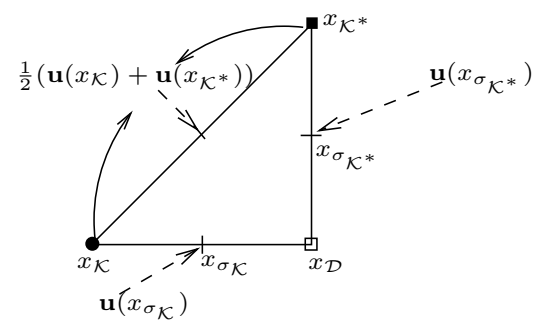

FIG. 6.1. The affine interpolation $\mathbb{P}_{c}^{\mathcal{Q}}$ on the quarter diamond cell $\mathcal{Q}=\mathcal{Q}_{\mathcal{K}, \mathcal{K}^{*}}$.

For instance, in the case of the quarter diamond cell $\mathcal{Q}=\mathcal{Q}_{\mathcal{K}, \mathcal{K}^{*}}$ (Fig. 6.1), it reads

$$
\mathbb{P}_{c}^{\mathcal{Q}} \mathbf{u}\left(x_{\sigma_{\mathcal{K}}}\right)=\mathbf{u}\left(x_{\sigma_{\mathcal{K}}}\right), \mathbb{P}_{c}^{\mathcal{Q}} \mathbf{u}\left(x_{\sigma_{\mathcal{K}^{*}}}\right)=\mathbf{u}\left(x_{\sigma_{\mathcal{K}^{*}}}\right), \mathbb{P}_{c}^{\mathcal{Q}} \mathbf{u}\left(\frac{x_{\mathcal{K}}+x_{\mathcal{K}^{*}}}{2}\right)=\frac{\mathbf{u}\left(x_{\mathcal{K}}\right)+\mathbf{u}\left(x_{\mathcal{K}^{*}}\right)}{2} .
$$

The following proposition is the vector-valued version of [5, inequality (5.4)] and can be proved exactly in the same way.

PROPOSITION 6.2. There exists a constant $C_{19}>0$, depending only on $\operatorname{reg}(\mathcal{T})$, such that for any function $\mathbf{v}$ in $\left(H^{2}(\mathfrak{Q})\right)^{2}$, we have for all $\mathcal{Q} \in \mathfrak{Q}$

$$
\int_{\mathcal{Q}}\left\|\nabla \mathbf{v}(z)-\nabla \mathbb{P}_{c}^{\mathcal{Q}} \mathbf{v}\right\|_{\mathcal{F}}^{2} \mathrm{~d} z+\int_{\mathcal{Q}}\left\|\mathrm{D} \mathbf{v}(z)-\mathrm{DP}_{c}^{\mathcal{Q}} \mathbf{v}\right\|_{\mathcal{F}}^{2} \mathrm{~d} z \leq C_{19} h_{\mathcal{D}}^{2} \int_{\mathcal{Q}}\left|\nabla^{2} \mathbf{v}(z)\right|^{2} \mathrm{~d} z
$$

6.4.3. Approximation of the viscous stress tensor. LEMMA 6.3. There exists a constant $C_{20}>0$, depending only on $\mathrm{C}_{\eta}, \overline{\mathrm{C}}_{\eta}$ and $\operatorname{reg}(\mathcal{T})$, such that for any function $\mathbf{u}$ in $\left(H^{2}(\mathfrak{Q})\right)^{2}$, we have for all $\mathcal{D} \in \mathfrak{D}$

$$
m_{\mathcal{Q}}\left|\mathbf{R}_{\mathfrak{s}, \mathcal{Q}}^{\mathbf{u}, \eta}\right|^{2} \leq C_{20} h_{\mathcal{D}}^{2} \int_{\mathcal{Q}}\left(\|\nabla \mathbf{u}\|_{\mathcal{F}}^{2}+\left|\nabla^{2} \mathbf{u}\right|^{2}\right) \mathrm{d} z, \quad \forall \mathcal{Q} \in \mathfrak{Q}_{\mathcal{D}}, \forall \mathfrak{s} \in \mathcal{E}_{\mathcal{Q}} .
$$

Proof. Applying the Jensen inequality, we have

$$
\left\|R_{\mathcal{Q}}^{\mathbf{u}, \eta}(z)\right\|_{\mathcal{F}}^{2} \leq \frac{1}{m_{\mathcal{Q}}} \int_{\mathcal{Q}}\|\eta(z) \operatorname{Du}(z)-\eta(x) \operatorname{Du}(x)\|_{\mathcal{F}}^{2} \mathrm{~d} x
$$

We add and subtract $\eta(z) \mathrm{Du}(x)$, Cauchy-Schwarz inequality implies that

$$
\left\|R_{\mathcal{Q}}^{\mathbf{u}, \eta}(z)\right\|_{\mathcal{F}}^{2} \leq \frac{2}{m_{\mathcal{Q}}} \int_{\mathcal{Q}}|\eta(z)-\eta(x)|^{2}\|\operatorname{Du}(x)\|_{\mathcal{F}}^{2} \mathrm{~d} x+\frac{2}{m_{\mathcal{Q}}} \int_{\mathcal{Q}}|\eta(z)|^{2}\|\mathrm{Du}(z)-\operatorname{Du}(x)\|_{\mathcal{F}}^{2} \mathrm{~d} x
$$


The assumption (1.2) and (2.1) give

$$
\left\|R_{\mathcal{Q}}^{\mathbf{u}, \eta}(z)\right\|_{\mathcal{F}}^{2} \leq \frac{2 \mathrm{C}_{\eta}^{2}}{m_{\mathcal{Q}}} h_{\mathcal{D}}^{2} \int_{\mathcal{Q}}\|\mathrm{D} \mathbf{u}(x)\|_{\mathcal{F}}^{2} \mathrm{~d} x+\frac{2 \overline{\mathrm{C}}_{\eta}^{2}}{m_{\mathcal{Q}}} \int_{\mathcal{Q}}\|\mathrm{Du}(z)-\operatorname{Du}(x)\|_{\mathcal{F}}^{2} \mathrm{~d} x .
$$

Since we have $\mathbf{R}_{\mathfrak{s}, \mathcal{Q}}^{\mathbf{u}, \eta}=\frac{1}{m_{\mathfrak{s}}} \int_{\mathfrak{s}} R_{\mathcal{Q}}^{\mathbf{u}, \eta}(z) \overrightarrow{\mathbf{n}}_{\mathfrak{s} \mathcal{Q}} \mathrm{d} z$, Jensen inequality implies that

$$
m_{\mathcal{Q}}\left|\mathbf{R}_{\mathfrak{s}, \mathcal{Q}}^{\mathbf{u}, \eta}\right|^{2} \leq h_{\mathcal{D}}^{2} 2 \mathrm{C}_{\eta}^{2} \int_{\mathcal{Q}}\|\operatorname{Du}(x)\|_{\mathcal{F}}^{2} \mathrm{~d} x+\frac{2 \overline{\mathrm{C}}_{\eta}^{2}}{m_{\mathfrak{s}}} \int_{\mathfrak{s}} \int_{\mathcal{Q}}\|\operatorname{Du}(z)-\operatorname{Du}(x)\|_{\mathcal{F}}^{2} \mathrm{~d} x \mathrm{~d} z .
$$

For the second integral, we apply Lemma 4.5 on a edge $\mathfrak{s}$ and the quarter diamond cell $\mathcal{Q}$, since $\frac{h_{\mathcal{Q}}^{3}}{m_{\mathfrak{s}}} \leq \operatorname{reg}(\mathcal{T}) h_{\mathcal{D}}^{2}$ :

$$
\frac{1}{m_{\mathfrak{s}}} \int_{\mathfrak{s}} \int_{\mathcal{Q}}\|\operatorname{Du}(z)-\operatorname{Du}(x)\|_{\mathcal{F}}^{2} \mathrm{~d} x \mathrm{~d} z \leq C_{11} \operatorname{reg}(\mathcal{T}) h_{\mathcal{D}}^{2} \int_{\mathcal{Q}}|\nabla \operatorname{Du}(y)|^{2} \mathrm{~d} y .
$$

Finally, we deduce the result with $C_{20}=\max \left(2 C_{\eta}^{2}, 2 \overline{\mathrm{C}}_{\eta}^{2} C_{11} \operatorname{reg}(\mathcal{T})\right)$.

6.4.4. Approximation of the gradient. Definition 6.4. We define $R^{\mathcal{D}} \in \mathcal{M}_{2,4}(\mathbb{R})$, for any $\mathcal{D} \in \mathfrak{D}$, as follows

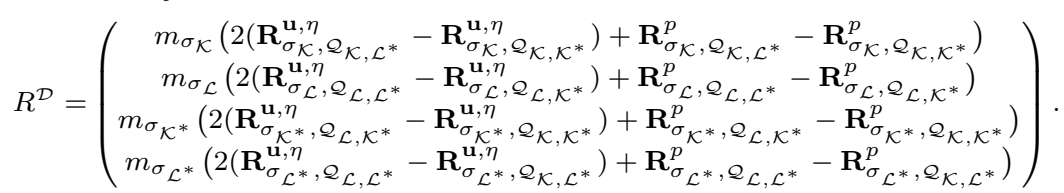

We also introduce the following norm for all $\mathcal{D} \in \mathfrak{D}$

$$
\|A\|_{\mathfrak{Q}_{\mathcal{D}}}^{2}=\sum_{\mathcal{Q} \in \mathfrak{Q}_{\mathcal{D}}}\|A\|_{L^{2}(\mathcal{Q})}^{2}, \quad \forall A_{\left.\right|_{\mathcal{Q}}} \in L^{2}\left(\mathcal{Q}, \mathcal{M}_{2}(\mathbb{R})\right), \text { for all } \mathcal{Q} \in \mathfrak{Q}_{\mathcal{D}}
$$

Proposition 6.3. There exists a constant $C_{21}>0$, depending only on $\underline{C}_{\eta}, \overline{\mathrm{C}}_{\eta}, \mathrm{C}_{\eta}$ and $\operatorname{reg}(\mathcal{T})$, such that for all $\mathcal{D} \in \mathfrak{D}$, such that for any $\delta^{\mathcal{D}}$ in $\mathcal{M}_{4,2}(\mathbb{R})$, we have

$\left|\operatorname{Tr}\left({ }^{t} \delta^{\mathcal{D}} R^{\mathcal{D}}\right)\right|^{2} \leq C_{21}\left(\sum_{\mathcal{Q} \in \mathfrak{Q}_{\mathcal{D}}} m_{\mathcal{Q}}\left\|B_{\mathcal{Q}} \delta^{\mathcal{D}}\right\|_{\mathcal{F}}^{2}\right) h_{\mathcal{D}}^{2} \sum_{\mathcal{Q} \in \mathfrak{Q}_{\mathcal{D}}} \int_{\mathcal{Q}}\left(\|\nabla \mathbf{u}(z)\|_{\mathcal{F}}^{2}+\left|\nabla^{2} \mathbf{u}(z)\right|^{2}+|\nabla p(z)|^{2}\right) \mathrm{d} z$.

Proof. We compute $\operatorname{Tr}\left({ }^{t} \delta^{\mathcal{D}} R^{\mathcal{D}}\right)$, then Cauchy-Schwarz inequality implies that

$$
\left|\operatorname{Tr}\left({ }^{t} \delta^{\mathcal{D}} R^{\mathcal{D}}\right)\right|^{2} \leq\left(\sum_{\mathcal{Q} \in \mathfrak{Q}_{\mathcal{D}}} m_{\mathcal{Q}}\left\|B_{\mathcal{Q}} \delta^{\mathcal{D}}\right\|_{\mathcal{F}}^{2}\right)\left(\sum_{\mathcal{Q} \in \mathfrak{Q}_{\mathcal{D}}} m_{\mathcal{Q}} \sum_{\mathfrak{s} \in \mathcal{E}_{\mathcal{Q}}}\left(\left|\mathbf{R}_{\mathfrak{s}, \mathcal{Q}}^{\mathbf{u}, \eta}\right|^{2}+\left|\mathbf{R}_{\mathfrak{s}, \mathcal{Q}}^{p}\right|^{2}\right)\right) .
$$

Lemmas 6.3 and 6.1 conclude the result.

The following proposition is proved in Section 9.3.

Proposition 6.4. We assume that $(\mathbf{u}, p)$ is the solution of the problem (1.1). For any $\mathcal{D} \in \mathfrak{D}$, there exists a constant $C_{22}>0$, depending only on $\underline{\mathrm{C}}_{\eta}, \overline{\mathrm{C}}_{\eta}$ and $\operatorname{reg}(\mathcal{T})$, and a function $\widetilde{\mathbf{v}}_{\mathcal{D}}$ which is an affine function on each $\mathcal{Q}$ of $\mathfrak{Q}_{\mathcal{D}}$ such that $\widetilde{\mathbf{v}}_{\mathcal{D}} \in\left(H^{1}(\mathcal{D})\right)^{2} \cap$ $\left(H^{2}(\mathcal{Q})\right)^{2}$, for all $\mathcal{Q} \in \mathfrak{Q}_{\mathcal{D}}$, and

$$
\left\|\mathrm{D} \widetilde{\mathbf{v}}_{\mathcal{D}}-\mathrm{D}_{\mathfrak{Q}}^{\mathcal{N}} \mathbb{P}_{\boldsymbol{c}}^{\mathcal{T}} \widetilde{\mathbf{v}}_{\mathcal{D}}\right\|_{\mathfrak{Q}_{\mathcal{D}}} \leq C_{22}\left(\left\|\widetilde{p}_{\mathrm{ex}}^{\mathfrak{Q}_{\mathcal{D}}}\right\|_{2}+\left\|\mathrm{Du}-\mathrm{D}_{\mathcal{Q}}^{\mathcal{N}} \mathbb{P}_{\boldsymbol{c}}^{\mathcal{T}} \mathbf{u}\right\|_{\mathfrak{Q}_{\mathcal{D}}}+h_{\mathcal{D}}\right),
$$

and

$$
-\sum_{\mathcal{Q} \in \mathfrak{Q}_{\mathcal{D}}} \int_{\mathcal{Q}} \operatorname{div} \widetilde{\mathbf{v}}_{\mathcal{D}} \widetilde{p}_{\mathrm{ex}}^{\mathcal{Q}} \mathrm{d} z \geq-C_{22}\left\|\mathrm{Du}-\mathrm{D}_{\mathcal{Q}}^{\mathcal{N}} \mathbb{P}_{\boldsymbol{c}}^{\mathcal{T}} \mathbf{u}\right\|_{\mathfrak{Q}_{\mathcal{D}}}^{2}+\frac{1}{2}\left\|\widetilde{p}_{\mathrm{ex}}^{\mathfrak{Q}_{\mathcal{D}}}\right\|_{2}^{2}-C_{22} \operatorname{size}(\mathcal{T}) .
$$


Remark that $\mathbb{P}_{c}^{\mathcal{T}} \widetilde{\mathbf{v}}_{\mathcal{D}}$, defined by (6.2), is not well defined, since $\widetilde{\mathbf{v}}_{\mathcal{D}}$ is discontinuous. Nevertheless, this function is only used locally on each diamond, thus $\mathbb{P}_{\boldsymbol{c}}^{\mathcal{T}} \widetilde{\mathbf{v}}_{\mathcal{D}}$ means in that case $\left(\widetilde{\mathbf{v}}_{\mathcal{D}}\left(x_{\mathcal{K}}\right), \widetilde{\mathbf{v}}_{\mathcal{D}}\left(x_{\mathcal{L}}\right), \widetilde{\mathbf{v}}_{\mathcal{D}}\left(x_{\mathcal{K}^{*}}\right), \widetilde{\mathbf{v}}_{\mathcal{D}}\left(x_{\mathcal{L}^{*}}\right)\right)$, for a diamond $\mathcal{D}$. We prove a consistency estimate for the modified strain rate tensor $D_{\mathfrak{Q}}^{\mathcal{N}}$ that we have introduced. This is the main difference between the present study and our previous work since the definition of the modified discrete strain rate tensor depends on the jumps of $\eta$ in each diamond cell. Hence, the consistency estimate for this operator cannot be obtained as in the usual way, that is, only by applying well-chosen Taylor formulae, we have to use here the fact that the pair $(\mathbf{u}, p)$ is a piecewisesmooth solution of the problem (1.1) and the estimate of Lemma 2.2. Note also that we can not prove separately the estimates on the velocity and on the pressure of the following lemma.

LEMMA 6.4. We assume that $(\mathbf{u}, p)$ is the solution of the problem (1.1). There exists a constant $C_{23}>0$, depending only on $\underline{\mathrm{C}}_{\eta}, \overline{\mathrm{C}}_{\eta}, \mathrm{C}_{\eta}, \operatorname{reg}(\mathcal{T})$ and $\sin \left(\epsilon_{0}\right)$, such that for all $\mathcal{D} \in \mathfrak{D}$, we have

$$
\left\|\mathrm{Du}-\mathrm{D}_{\mathfrak{Q}}^{\mathcal{N}} \mathbb{P}_{\boldsymbol{c}}^{\mathcal{T}} \mathbf{u}\right\|_{\mathfrak{Q}_{\mathcal{D}}}^{2}+\left\|\widetilde{p}_{\mathrm{ex}}^{\mathfrak{Q}_{\mathcal{D}}}\right\|_{2}^{2} \leq C_{23} h_{\mathcal{D}}^{2} \sum_{\mathcal{Q} \in \mathfrak{Q}_{\mathcal{D}}} \int_{\mathcal{Q}}\left(\|\nabla \mathbf{u}\|_{\mathcal{F}}^{2}+\left|\nabla^{2} \mathbf{u}\right|^{2}+|\nabla p(z)|^{2}\right) \mathrm{d} z
$$

Proof. Let us give the proof in the case where the diamond cell $\mathcal{D}$ is an interior diamond cell. The case $\mathcal{D} \in \mathfrak{D}_{\text {ext }}$ can be treated in a same way.

Step 1. Since $(\mathbf{u}, p)$ solves (1.1), we have the conservativity of the fluxes through $\mathfrak{s}=$ $\mathcal{Q} \mid \mathcal{Q}^{\prime}$ as follows

$$
\int_{\mathfrak{s}}\left(2 \eta_{\mid \overline{\mathcal{Q}}}(z) \mathrm{D} \mathbf{u}_{\mid \overline{\mathcal{Q}}}(z)-p_{\mid \overline{\mathcal{Q}}}(z) \operatorname{Id}\right) \overrightarrow{\mathbf{n}}_{\mathfrak{s} \mathcal{Q}} \mathrm{d} z=\int_{\mathfrak{s}}\left(2 \eta_{\mid \overline{\mathcal{Q}^{\prime}}}(z) \operatorname{D\mathbf {u}_{|\overline {\mathcal {Q}^{\prime }}}}(z)-p_{\mid \overline{\mathcal{Q}^{\prime}}}(z) \operatorname{Id}\right) \overrightarrow{\mathbf{n}}_{\mathfrak{s} \mathcal{Q}} \mathrm{d} z
$$

We recall that the discrete strain rate tensor satisfies Proposition 6.1, we can deduce that

$$
\begin{aligned}
& \left(\frac{1}{m_{\mathfrak{s}}} \int_{\mathfrak{s}}\left(2 \eta_{\mid \overline{\mathcal{Q}}}(z) \mathrm{D} \mathbf{u}_{\mid \overline{\mathcal{Q}}}(z)-p_{\mid \overline{\mathcal{Q}}}(z) \mathrm{Id}\right) \mathrm{d} z-\left(2 \eta_{\mathcal{Q}} \mathrm{D}_{\mathcal{Q}}^{\mathcal{N}} \mathbb{P}_{\boldsymbol{c}}^{\mathcal{T}} \mathbf{u}-p_{\text {ex }}^{\mathcal{Q}} \mathrm{Id}\right)\right) \overrightarrow{\mathbf{n}}_{\mathfrak{s} \mathcal{Q}} \\
& \quad-\left(\frac{1}{m_{\mathfrak{s}}} \int_{\mathfrak{s}}\left(2 \eta_{\mid \mathcal{Q}^{\prime}}(z) \mathrm{D} \mathbf{u}_{\mid \overline{\mathcal{Q}^{\prime}}}(z)-p_{\mid \overline{\mathcal{Q}^{\prime}}}(z) \mathrm{Id}\right) \mathrm{d} z-\left(2 \eta_{\mathcal{Q}^{\prime}} \mathrm{D}_{\mathcal{Q}^{\prime}}^{\mathcal{N}} \mathbb{P}_{\boldsymbol{c}}^{\mathcal{T}} \mathbf{u}-p_{\mathrm{ex}}^{\mathcal{Q}^{\prime}} \mathrm{Id}\right)\right) \overrightarrow{\mathbf{n}}_{\mathfrak{s} \mathcal{Q}}=0 .
\end{aligned}
$$

Using Definition 6.1 and the last equality, we have

$$
\begin{gathered}
\frac{2}{m_{\mathcal{Q}}} \int_{\mathcal{Q}} \eta(z)\left(\operatorname{Du}(z)-\mathrm{D}_{\mathcal{Q}}^{\mathcal{N}} \mathbb{P}_{\boldsymbol{c}}^{\mathcal{T}} \mathbf{u}\right) \overrightarrow{\mathbf{n}}_{\mathfrak{s} \mathcal{Q}} \mathrm{d} z-\frac{2}{m_{\mathcal{Q}^{\prime}}} \int_{\mathcal{Q}^{\prime}} \eta(z)\left(\mathrm{Du}(z)-\mathrm{D}_{\mathcal{Q}^{\prime}}^{\mathcal{N}} \mathbb{P}_{\boldsymbol{c}}^{\mathcal{T}} \mathbf{u}\right) \overrightarrow{\mathbf{n}}_{\mathfrak{s} \mathcal{Q}} \mathrm{d} z \\
-\frac{1}{m_{\mathcal{Q}}} \int_{\mathcal{Q}}\left(p(z)-p_{\mathrm{ex}}^{\mathcal{Q}}\right) \overrightarrow{\mathbf{n}}_{\mathfrak{s} \mathcal{Q}} \mathrm{d} z+\frac{1}{m_{\mathcal{Q}^{\prime}}} \int_{\mathcal{Q}^{\prime}}\left(p(z)-p_{\mathrm{ex}}^{\mathcal{Q}^{\prime}}\right) \overrightarrow{\mathbf{n}}_{\mathfrak{s} \mathcal{Q}} \mathrm{d} z \\
=2 \mathbf{R}_{\mathfrak{s}, \mathcal{Q}^{\prime}}^{\mathbf{u}, \eta}+2 \mathbf{R}_{\mathfrak{s}, \mathcal{Q}^{\prime}}^{\mathbf{u}, z}-2 \mathbf{R}_{\mathfrak{s}, \mathcal{Q}}^{\mathbf{u}, \eta}-2 \mathbf{R}_{\mathfrak{s}, \mathcal{Q}}^{\mathbf{u}, z}-\mathbf{R}_{\mathfrak{s}, \mathcal{Q}}^{p}+\mathbf{R}_{\mathfrak{s}, \mathcal{Q}^{\prime}}^{p}
\end{gathered}
$$

We sum over the quarter diamond cells $\mathcal{Q} \in \mathfrak{Q}_{\mathcal{D}}$

$$
\sum_{\mathcal{Q} \in \mathfrak{Q}_{\mathcal{D}}}\left(\int_{\mathcal{Q}} 2 \eta(z)\left(\mathrm{Du}(z)-\mathrm{D}_{\mathcal{Q}}^{\mathcal{N}} \mathbb{P}_{c}^{\mathcal{T}} \mathbf{u}\right) \mathrm{d} z\right) B_{\mathcal{Q}}-\sum_{\mathcal{Q} \in \mathfrak{Q}_{\mathcal{D}}}\left(\int_{\mathcal{Q}}\left(p(z)-p_{\text {ex }}^{\mathcal{Q}}\right) \mathrm{d} z\right) B_{\mathcal{Q}}={ }^{t} R^{\mathcal{D}}
$$

with $R^{\mathcal{D}}$ defined by Definition 6.4. We multiply (6.11) by any $\delta^{\mathcal{D}} \in \mathcal{M}_{n_{\mathcal{D}}, 2}(\mathbb{R})$ and take the transpose, thanks to the symmetry of $\operatorname{Du}(z)$ and $\mathrm{D}_{\mathcal{Q}}^{\mathcal{N}} \mathbb{P}_{c}^{\mathcal{T}} \mathbf{u}$, we obtain

$$
\sum_{\mathcal{Q} \in \mathfrak{Q}_{\mathcal{D}}} \int_{\mathcal{Q}}^{t} \delta^{\mathcal{D} t} B_{\mathcal{Q}} 2 \eta(z)\left(\mathrm{Du}(z)-\mathrm{D}_{\mathcal{Q}}^{\mathcal{N}} \mathbb{P}_{\boldsymbol{c}}^{\mathcal{T}} \mathbf{u}\right) \mathrm{d} z-\sum_{\mathcal{Q} \in \mathfrak{Q}_{\mathcal{D}}} m_{\mathcal{Q}}{ }^{t} \delta^{\mathcal{D} t} B_{\mathcal{Q}} \widetilde{p}_{\mathrm{ex}}^{\mathcal{Q}}={ }^{t} \delta^{\mathcal{D}} R^{\mathcal{D}}
$$


Using the trace operator we deduce

$$
\begin{aligned}
\sum_{\mathcal{Q} \in \mathfrak{Q}_{\mathcal{D}}} \int_{\mathcal{Q}} \eta(z) & \left(B_{\mathcal{Q}} \delta^{\mathcal{D}}+{ }^{t} \delta^{\mathcal{D} t} B_{\mathcal{Q}}: \operatorname{Du}(z)-\mathrm{D}_{\mathcal{Q}}^{\mathcal{N}} \mathbb{P}_{c}^{\mathcal{T}} \mathbf{u}\right) \mathrm{d} z \\
& -\sum_{\mathcal{Q} \in \mathfrak{Q}_{\mathcal{D}}} m_{\mathcal{Q}} \operatorname{Tr}\left({ }^{t} \delta^{\mathcal{D} t} B_{\mathcal{Q}}\right) \widetilde{p}_{\mathrm{ex}}^{\mathcal{Q}}=\operatorname{Tr}\left({ }^{t} \delta^{\mathcal{D}} R^{\mathcal{D}}\right), \quad \forall \delta^{\mathcal{D}} \in \mathcal{M}_{n_{\mathcal{D}}, 2}(\mathbb{R}) .
\end{aligned}
$$

Step 2. For $\mathbf{u}, \mathbf{v} \in\left(H^{2}(\mathfrak{Q})\right)^{2}$, and $p^{\mathfrak{Q}}, q^{\mathfrak{Q}} \in \mathbb{R}^{\mathfrak{Q}}$, we define a bilinear form $\mathcal{B}_{\mathcal{D}}$ as follows

$$
\begin{aligned}
\mathcal{B}_{\mathcal{D}}\left(\mathbf{u}, p^{\mathfrak{Q}}, \mathbf{v}, q^{\mathfrak{Q}}\right) & =\sum_{\mathcal{Q} \in \mathfrak{Q}_{\mathcal{D}}} 2 \int_{\mathcal{Q}} \eta(z)\left(\mathrm{D} \mathbf{u}-\mathrm{D}_{\mathcal{Q}}^{\mathcal{N}} \mathbb{P}_{\boldsymbol{c}}^{\mathcal{T}} \mathbf{u}: \mathrm{D} \mathbf{v}-\mathrm{D}_{\mathcal{Q}}^{\mathcal{N}} \mathbb{P}_{\boldsymbol{c}}^{\mathcal{T}} \mathbf{v}\right) \mathrm{d} z \\
& -\sum_{\mathcal{Q} \in \mathfrak{Q}_{\mathcal{D}}} \int_{\mathcal{Q}} \operatorname{Tr}\left(\mathrm{D} \mathbf{v}-\mathrm{D}_{\mathcal{Q}}^{\mathcal{N}} \mathbb{P}_{\boldsymbol{c}}^{\mathcal{T}} \mathbf{v}\right) p^{\mathcal{Q}} \mathrm{d} z+\sum_{\mathcal{Q} \in \mathfrak{Q}_{\mathcal{D}}} \int_{\mathcal{Q}} \operatorname{Tr}\left(\mathrm{Du}-\mathrm{D}_{\mathcal{Q}}^{\mathcal{N}} \mathbb{P}_{c}^{\mathcal{T}} \mathbf{u}\right) q^{\mathcal{Q}} \mathrm{d} z .
\end{aligned}
$$

We easily have that

$$
\mathcal{B}_{\mathcal{D}}\left(\mathbf{u}, \widetilde{p}_{\mathrm{ex}}^{\mathfrak{Q}_{\mathcal{D}}}, \mathbf{u}, \widetilde{p}_{\mathrm{ex}}^{\mathfrak{Q}_{\mathcal{D}}}\right) \geq 2 \underline{\mathrm{C}}_{\eta}\left\|\mathrm{D} \mathbf{u}-\mathrm{D}_{\mathcal{Q}}^{\mathcal{N}} \mathbb{P}_{\boldsymbol{c}}^{\mathcal{T}} \mathbf{u}\right\|_{\mathfrak{Q}_{\mathcal{D}}}^{2}
$$

Thanks to Cauchy-Schwarz inequality, using the function $\widetilde{\mathbf{v}}_{\mathcal{D}}$ obtained in Proposition 6.4, we have

$$
\begin{aligned}
\mathcal{B}_{\mathcal{D}}\left(\mathbf{u}, \widetilde{p}_{\mathrm{ex}}^{\mathfrak{Q}_{\mathcal{D}}}, \widetilde{\mathbf{v}}_{\mathcal{D}}, 0\right) \geq & -2 \overline{\mathrm{C}}_{\eta}\left\|\mathrm{Du}-\mathrm{D}_{\mathcal{Q}}^{\mathcal{N}} \mathbb{P}_{\boldsymbol{c}}^{\mathcal{T}} \mathbf{u}\right\|_{\mathfrak{Q}_{\mathcal{D}}}\left\|\mathrm{D} \widetilde{\mathbf{v}}_{\mathcal{D}}-\mathrm{D}_{\mathcal{Q}}^{\mathcal{N}} \mathbb{P}_{\boldsymbol{c}}^{\mathcal{T}} \widetilde{\mathbf{v}}_{\mathcal{D}}\right\|_{\mathfrak{Q}_{\mathcal{D}}} \\
& -\sum_{\mathcal{Q} \in \mathfrak{Q}_{\mathcal{D}}} \int_{\mathcal{Q}} \operatorname{div} \widetilde{\mathbf{v}}_{\mathcal{D}} \widetilde{p}_{\text {ex }}^{\mathcal{Q}} \mathrm{d} z+\sum_{\mathcal{Q} \in \mathfrak{Q}_{\mathcal{D}}} m_{\mathcal{Q}} \operatorname{Tr}\left(\mathrm{D}_{\mathcal{Q}}^{\mathcal{N}} \mathbb{P}_{\boldsymbol{c}}^{\mathcal{T}} \widetilde{\mathbf{v}}_{\mathcal{D}}\right) \widetilde{p}_{\text {ex }}^{\mathcal{Q}} .
\end{aligned}
$$

For the last term of the above estimate, since we have $\operatorname{Tr}\left(\mathrm{D}_{\mathcal{Q}}^{\mathcal{N}} \mathbb{P}_{\boldsymbol{c}}^{\mathcal{T}} \widetilde{\mathbf{v}}_{\mathcal{D}}\right)=\operatorname{div}^{\mathcal{D}}\left(\mathbb{P}_{\boldsymbol{c}}^{\mathcal{T}} \widetilde{\mathbf{v}}_{\mathcal{D}}\right)$ (see (2.19)), for all $\mathcal{Q} \in \mathfrak{Q}_{\mathcal{D}}$, we have $\sum_{\mathcal{Q} \in \mathfrak{Q}_{\mathcal{D}}} m_{\mathcal{Q}} \operatorname{Tr}\left(\mathrm{D}_{\mathcal{Q}}^{\mathcal{N}} \mathbb{P}_{\boldsymbol{c}}^{\mathcal{T}} \widetilde{\mathbf{v}}_{\mathcal{D}}\right) \widetilde{p}_{\text {ex }}^{\mathcal{Q}}=\operatorname{div}^{\mathcal{D}}\left(\mathbb{P}_{\boldsymbol{c}}^{\mathcal{T}} \widetilde{\mathbf{v}}_{\mathcal{D}}\right) \sum_{\mathcal{Q} \in \mathfrak{Q}_{\mathcal{D}}} m_{\mathcal{Q}} \widetilde{p}_{\mathrm{ex}}^{\mathcal{Q}}$, thanks to (6.3), we deduce that $\sum_{\mathcal{Q} \in \mathfrak{Q}_{\mathcal{D}}} m_{\mathcal{Q}} \operatorname{Tr}\left(\mathrm{D}_{\mathcal{Q}}^{\mathcal{N}} \mathbb{P}_{\boldsymbol{c}}^{\mathcal{T}} \widetilde{\mathbf{v}}_{\mathcal{D}}\right) \widetilde{p}_{\text {ex }}^{\mathcal{Q}}=0$. Finally, the estimate on $\widetilde{\mathbf{v}}_{\mathcal{D}}$ in Proposition 6.4 and Young inequality conclude that

$$
\mathcal{B}_{\mathcal{D}}\left(\mathbf{u}, \widetilde{p}_{\mathrm{ex}}^{\mathfrak{Q}_{\mathcal{D}}}, \widetilde{\mathbf{v}}_{\mathcal{D}}, 0\right) \geq-C\left\|\mathrm{Du}-\mathrm{D}_{\mathcal{Q}}^{\mathcal{N}} \mathbb{P}_{\boldsymbol{c}}^{\mathcal{T}} \mathbf{u}^{2}{\underline{\mathfrak{Q}_{\mathcal{D}}}}^{2}+\frac{1}{4}\right\| \widetilde{p}_{\mathrm{ex}}^{\mathfrak{Q}_{\mathcal{D}}} \|_{2}^{2}-C \operatorname{size}(\mathcal{T})^{2}
$$

By bilinearity of $\mathcal{B}_{\mathcal{D}}$, the inequalities (6.13) and (6.14) give for each positive number $\xi>0$ :

$\mathcal{B}_{\mathcal{D}}\left(\mathbf{u}, \widetilde{p}_{\mathrm{ex}}^{\mathfrak{Q}_{\mathcal{D}}} ; \mathbf{u}+\xi \widetilde{\mathbf{v}}_{\mathcal{D}}, \widetilde{p}_{\mathrm{ex}}^{\mathfrak{Q}_{\mathcal{D}}}\right)+\xi C \operatorname{size}(\mathcal{T})^{2} \geq\left(2 \underline{\mathrm{C}}_{\eta}-\xi C\right)\left\|\mathrm{Du}-\mathrm{D}_{\mathcal{Q}}^{\mathcal{N}} \mathbb{P}_{\boldsymbol{c}}^{\mathcal{T}} \mathbf{u}\right\|_{\mathfrak{Q}_{\mathcal{D}}}^{2}+\frac{\xi}{2}\left\|\widetilde{p}_{\text {ex }}^{\mathfrak{Q}_{\mathcal{D}}}\right\|_{2}^{2}$.

Choosing a value of $\xi>0$ small enough (depending only on $\underline{\mathrm{C}}_{\eta}$ and $\overline{\mathrm{C}}_{\eta}$ ), the above inequality yields the following estimates for $\overline{\mathbf{u}}=\mathbf{u}+\xi \widetilde{\mathbf{v}}_{\mathcal{D}} \in\left(H^{2}(\mathfrak{Q})\right)^{2}$

$$
\left\|\mathrm{D} \overline{\mathbf{u}}-\mathrm{D}_{\mathcal{Q}}^{\mathcal{N}} \mathbb{P}_{\boldsymbol{c}}^{\mathcal{T}} \overline{\mathbf{u}}\right\|_{\mathfrak{Q}_{\mathcal{D}}} \leq C\left(\left\|\mathrm{D} \mathbf{u}-\mathrm{D}_{\mathcal{Q}}^{\mathcal{N}} \mathbb{P}_{\boldsymbol{c}}^{\mathcal{T}} \mathbf{u}\right\|_{\mathfrak{Q}_{\mathcal{D}}}+\left\|\widetilde{p}_{\mathrm{ex}}^{\mathfrak{Q}_{\mathcal{D}}}\right\|_{2}+h_{\mathcal{D}}\right)
$$

and

$$
\left\|\mathrm{Du}-\mathrm{D}_{\mathcal{Q}}^{\mathcal{N}} \mathbb{P}_{\boldsymbol{c}}^{\mathcal{T}} \mathbf{u}\right\|_{\mathfrak{Q}_{\mathcal{D}}}^{2}+\left\|\widetilde{p}_{\mathrm{ex}}^{\mathfrak{Q}_{\mathcal{D}}}\right\|_{2}^{2} \leq C \mathcal{B}_{\mathcal{D}}\left(\mathbf{u}, \widetilde{p}_{\mathrm{ex}}^{\mathfrak{Q}_{\mathcal{D}}} ; \overline{\mathbf{u}}, \widetilde{p}_{\mathrm{ex}}^{\mathfrak{Q}_{\mathcal{D}}}\right)+C_{3} \operatorname{size}(\mathcal{T})^{2}
$$

Step 3. We define now the consistency error for the projection $\mathbb{P}_{c}^{\mathfrak{Q}}$ as follows

$$
T_{\overline{\mathcal{Q}}, \overline{\mathbf{u}}}(z)=\mathrm{D} \overline{\mathbf{u}}(z)-\mathrm{DP}_{c}^{\mathcal{Q}} \overline{\mathbf{u}}, \quad \forall z \in \mathcal{Q}, \quad \forall \mathcal{Q} \in \mathfrak{Q}
$$


Remark that $2 \mathrm{DP}_{c}^{\mathcal{Q}} \overline{\mathbf{u}}-2 \mathrm{D}^{\mathcal{D}} \mathbb{P}_{c}^{\mathcal{T}} \overline{\mathbf{u}}$ satisfied the conditions (2.13) and (2.14) if $\alpha_{\mathcal{K}}=\alpha_{\mathcal{L}}$, thanks to Proposition 2.2 , there exists a unique $\widetilde{\delta^{\mathcal{D}}} \in \mathcal{M}_{n_{\mathcal{D}}, 2}(\mathbb{R})$ such that $\left(\widetilde{\delta^{\mathcal{D}}}: \delta_{0}\right)=0$ (with $\delta_{0}$ defined in Proposition 2.1) and

$$
\mathrm{DP}_{c}^{\mathcal{Q}} \overline{\mathbf{u}}-\mathrm{D}^{\mathcal{D}} \mathbb{P}_{\boldsymbol{c}}^{\mathcal{T}} \overline{\mathbf{u}}=\frac{1}{2}\left(B_{\mathcal{Q}} \widetilde{\delta^{\mathcal{D}}}+{ }^{t} \widetilde{\delta^{\mathcal{D}}} t B_{\mathcal{Q}}\right), \quad \forall \mathcal{Q} \in \mathfrak{Q}_{\mathcal{D}}
$$

Then applying Theorem 2.1 with $\left(\mathrm{D}^{\mathcal{D}} \mathbb{P}_{\boldsymbol{c}}^{\mathcal{T}} \overline{\mathbf{u}}, \mathbb{P}_{m}^{\mathfrak{D}} p\right)$, there exists a unique pair $\left(\overline{\delta^{\mathcal{D}}}, \overline{p^{\mathfrak{Q}_{\mathcal{D}}}}\right) \in$ $\mathcal{M}_{n_{\mathcal{D}}, 2}(\mathbb{R}) \times \mathbb{R}^{n_{\mathcal{D}}}$ satisfying (2.15). So we have $\mathrm{D}_{\mathcal{Q}}^{\mathcal{N}} \mathbb{P}_{\boldsymbol{c}}^{\mathcal{T}} \overline{\mathbf{u}}=\mathrm{D}^{\mathcal{D}} \mathbb{P}_{\boldsymbol{c}}^{\mathcal{T}} \overline{\mathbf{u}}+\frac{1}{2}\left(B_{\mathcal{Q}} \overline{\delta^{\mathcal{D}}}+{ }^{t} \overline{\delta^{\mathcal{D}}} B_{\mathcal{Q}}\right)$, with $\left(\overline{\delta^{\mathcal{D}}}: \delta_{0}\right)=0$. We note now $\widehat{\delta^{\mathcal{D}}}=\widetilde{\delta^{\mathcal{D}}}-\overline{\delta^{\mathcal{D}}}$ which satisfies $\left(\widehat{\delta^{\mathcal{D}}}: \delta_{0}\right)=0$ and

$$
\mathrm{DP}_{c}^{\mathcal{Q}} \overline{\mathbf{u}}-\mathrm{D}_{\mathcal{Q}}^{\mathcal{N}} \mathbb{P}_{c}^{\mathcal{I}} \overline{\mathbf{u}}=\frac{1}{2}\left(B_{\mathcal{Q}} \widehat{\delta^{\mathcal{D}}}+{ }^{t}{\widehat{\delta^{\mathcal{D}}}}^{t} B_{\mathcal{Q}}\right), \quad \forall \mathcal{Q} \in \mathfrak{Q}_{\mathcal{D}}
$$

Replacing $\delta^{\mathcal{D}}$ by $\widehat{\delta^{\mathcal{D}}}$ in (6.12) and using the fact that

$$
\frac{1}{2}\left(B_{\mathcal{Q}} \widehat{\delta^{\mathcal{D}}}+{ }^{t} \widehat{\delta^{\mathcal{D}} t} B_{\mathcal{Q}}\right)=\mathrm{DP}_{c}^{\mathcal{Q}} \overline{\mathbf{u}}-\mathrm{D}_{\mathcal{Q}}^{\mathcal{N}} \mathbb{P}_{\boldsymbol{c}}^{\mathcal{T}} \overline{\mathbf{u}}=\mathrm{D} \overline{\mathbf{u}}(z)-\mathrm{D}_{\mathcal{Q}}^{\mathcal{N}} \mathbb{P}_{\boldsymbol{c}}^{\mathcal{T}} \overline{\mathbf{u}}-T_{\overline{\mathcal{Q}}, \overline{\mathbf{u}}}(z),
$$

we deduce that

$$
\begin{array}{r}
\operatorname{Tr}\left({ }^{t} \widehat{\delta^{\mathcal{D}}} R^{\mathcal{D}}\right)=\sum_{\mathcal{Q} \in \mathfrak{Q}_{\mathcal{D}}} \int_{\mathcal{Q}} 2 \eta(z)\left(\mathrm{D} \overline{\mathbf{u}}(z)-\mathrm{D}_{\mathcal{Q}}^{\mathcal{N}} \mathbb{P}_{\boldsymbol{c}}^{\mathcal{T}} \overline{\mathbf{u}}: \operatorname{Du}(z)-\mathrm{D}_{\mathcal{Q}}^{\mathcal{N}} \mathbb{P}_{\boldsymbol{c}}^{\mathcal{T}} \mathbf{u}\right) \mathrm{d} z+\sum_{\mathcal{Q} \in \mathfrak{Q}_{\mathcal{D}}} m_{\mathcal{Q}} \operatorname{Tr}\left(T_{\overline{\mathcal{Q}}, \overline{\mathbf{u}}}(z)\right) \widetilde{p}_{\mathrm{ex}}^{\mathcal{Q}} \\
\quad-\sum_{\mathcal{Q} \in \mathfrak{Q}_{\mathcal{D}}} \int_{\mathcal{Q}} 2 \eta(z)\left(T_{\overline{\mathcal{Q}}, \overline{\mathbf{u}}}(z): \operatorname{Du}(z)-\mathrm{D}_{\mathcal{Q}}^{\mathcal{N}} \mathbb{P}_{\boldsymbol{c}}^{\mathcal{T}} \mathbf{u}\right) \mathrm{d} z-\sum_{\mathcal{Q} \in \mathfrak{Q}_{\mathcal{D}}} m_{\mathcal{Q}} \operatorname{Tr}\left(\operatorname{Dr} \overline{\mathbf{u}}(z)-\mathrm{D}_{\mathcal{Q}}^{\mathcal{N}} \mathbb{P}_{\boldsymbol{c}}^{\mathcal{T}} \overline{\mathbf{u}}\right) \widetilde{p}_{\mathrm{ex}}^{\mathcal{Q}} .
\end{array}
$$

Now we can link $R^{\mathcal{D}}$ and $\mathcal{B}_{\mathcal{D}}$ as follows

$$
\begin{aligned}
& \mathcal{B}_{\mathcal{D}}\left(\mathbf{u}, \widetilde{p}_{\mathrm{ex}}^{\mathfrak{Q}_{\mathcal{D}}} ; \overline{\mathbf{u}}, \widetilde{p}_{\mathrm{ex}}^{\mathfrak{Q}_{\mathcal{D}}}\right)=\operatorname{Tr}\left({ }^{t} \widehat{\delta^{\mathcal{D}}} R^{\mathcal{D}}\right)-\sum_{\mathcal{Q} \in \mathfrak{Q}_{\mathcal{D}}} m_{\mathcal{Q}} \operatorname{Tr}\left(T_{\overline{\mathcal{Q}}, \overline{\mathbf{u}}}(z)\right) \widetilde{p}_{\mathrm{ex}}^{\mathcal{Q}} \\
& \quad+\sum_{\mathcal{Q} \in \mathfrak{Q}_{\mathcal{D}}} \int_{\mathcal{Q}} 2 \eta(z)\left(T_{\overline{\mathcal{Q}}, \overline{\mathbf{u}}}(z): \operatorname{Du}(z)-\mathrm{D}_{\mathcal{Q}}^{\mathcal{N}} \mathbb{P}_{\boldsymbol{c}}^{\mathcal{T}} \mathbf{u}\right) \mathrm{d} z+\sum_{\mathcal{Q} \in \mathfrak{Q}_{\mathcal{D}}} m_{\mathcal{Q}} \operatorname{Tr}\left(\operatorname{Du}(z)-\mathrm{D}_{\mathcal{Q}}^{\mathcal{N}} \mathbb{P}_{\boldsymbol{c}}^{\mathcal{T}} \mathbf{u}\right) \widetilde{p}_{\mathrm{ex}}^{\mathcal{Q}} .
\end{aligned}
$$

Thanks to (1.2) and the Cauchy-Schwarz inequality, Proposition 6.3 implies that

$$
\begin{aligned}
& \mathcal{B}_{\mathcal{D}}\left(\mathbf{u}, \widetilde{p}_{\mathrm{ex}}^{\mathfrak{Q}_{\mathcal{D}}} ; \overline{\mathbf{u}}, \widetilde{p}_{\mathrm{ex}}^{\mathfrak{Q}_{\mathcal{D}}}\right) \leq C h_{\mathcal{D}}\left\|B_{\mathcal{Q}} \widehat{\delta^{\mathcal{D}}}\right\|_{\mathfrak{Q}_{\mathcal{D}}}\left(\sum_{\mathcal{Q} \in \mathfrak{Q}_{\mathcal{D}}} \int_{\mathcal{Q}}\left(\|\nabla \mathbf{u}(z)\|_{\mathcal{F}}^{2}+\left|\nabla^{2} \mathbf{u}(z)\right|^{2}+|\nabla p(z)|^{2}\right) \mathrm{d} z\right)^{\frac{1}{2}} \\
& \quad+\left\|T_{\overline{\mathfrak{Q}}, \overline{\mathbf{u}}}\right\|_{\mathfrak{Q}_{\mathcal{D}}}\left\|\widetilde{p}_{\mathrm{ex}}^{\mathfrak{Q}_{\mathcal{D}}}\right\|_{2}+2 \overline{\mathrm{C}}_{\eta}\left\|\mathrm{D} \mathbf{u}-\mathrm{D}_{\mathfrak{Q}}^{\mathcal{N}} \mathbb{P}_{\boldsymbol{c}}^{\mathcal{T}} \mathbf{u}\right\|_{\mathfrak{Q}_{\mathcal{D}}}\left\|T_{\overline{\mathfrak{Q}}, \overline{\mathbf{u}}}\right\|_{\mathfrak{Q}_{\mathcal{D}}}+\left\|\mathrm{D} \mathbf{u}-\mathrm{D}_{\mathcal{Q}}^{\mathcal{N}} \mathbb{P}_{\boldsymbol{c}}^{\mathcal{T}} \mathbf{u}\right\|_{\mathfrak{Q}_{\mathcal{D}}}\left\|\widetilde{p}_{\mathrm{ex}}^{\mathfrak{Q}_{\mathcal{D}}}\right\|_{2} .
\end{aligned}
$$

Thanks to Lemma 2.2 and the estimate (6.18), we have

$$
\sum_{\mathcal{Q} \in \mathfrak{Q}_{\mathcal{D}}} m_{\mathcal{Q}}\left\|B_{\mathcal{Q}} \widehat{\delta^{\mathcal{D}}}\right\|_{\mathcal{F}}^{2} \leq C\left(\left\|\mathrm{D} \overline{\mathbf{u}}-\mathrm{D}_{\mathfrak{Q}}^{\mathcal{N}} \mathbb{P}_{\boldsymbol{c}}^{\mathcal{T}} \overline{\mathbf{u}}\right\|_{\mathfrak{Q}_{\mathcal{D}}}^{2}+\left\|T_{\overline{\mathfrak{Q}}, \overline{\mathbf{u}}}\right\|_{\mathfrak{Q}_{\mathcal{D}}}^{2}\right)
$$

Using (6.15) and (6.16), Proposition 6.2 implies

$$
\begin{aligned}
& \left\|\mathrm{D} \mathbf{u}-\mathrm{D}_{\mathcal{Q}}^{\mathcal{N}} \mathbb{P}_{\boldsymbol{c}}^{\mathcal{T}} \mathbf{u}\right\|_{\mathfrak{Q}_{\mathcal{D}}}^{2}+\left\|\widetilde{p}_{\text {ex }}^{\mathfrak{Q}_{\mathcal{D}}}\right\|_{2}^{2} \leq C h_{\mathcal{D}}^{2} \sum_{\mathcal{Q} \in \mathfrak{Q}_{\mathcal{D}}} \int_{\mathcal{Q}}\left(\|\nabla \mathbf{u}(z)\|_{\mathcal{F}}^{2}+\left|\nabla^{2} \mathbf{u}(z)\right|^{2}+|\nabla p(z)|^{2}\right) \mathrm{d} z \\
& +C h_{\mathcal{D}}\left(\left\|\mathrm{D} \mathbf{u}-\mathrm{D}_{\mathfrak{Q}}^{\mathcal{N}} \mathbb{P}_{\boldsymbol{c}}^{\mathcal{T}} \mathbf{u}\right\|_{\mathfrak{Q}_{\mathcal{D}}}+\left\|\widetilde{p}_{\mathrm{ex}}^{\mathfrak{Q}_{\mathcal{D}}}\right\|_{2}\right)\left(\sum_{\mathcal{Q} \in \mathfrak{Q}_{\mathcal{D}}} \int_{\mathcal{Q}}\left(\|\nabla \mathbf{u}(z)\|_{\mathcal{F}}^{2}+\left|\nabla^{2} \mathbf{u}(z)\right|^{2}+|\nabla p(z)|^{2}\right) \mathrm{d} z\right)^{\frac{1}{2}} \\
& +\left\|\mathrm{Du}-\mathrm{D}_{\mathcal{Q}}^{\mathcal{N}} \mathbb{P}_{c}^{\mathcal{T}} \mathbf{u}\right\|_{\mathfrak{Q}_{\mathcal{D}}}\left\|\widetilde{p}_{\mathrm{ex}}^{\mathfrak{Q}_{\mathcal{D}}}\right\|_{2}
\end{aligned}
$$

Finally, Young inequality gives the result. 
REMARK 6.1. We immediately have the estimate on the whole norm for $(\mathbf{u}, p)$

$$
\left\|\mathrm{Du}-\mathrm{D}_{\mathfrak{Q}}^{\mathcal{N}} \mathbb{P}_{\boldsymbol{c}}^{\mathcal{T}} \mathbf{u}\right\|_{2}^{2}+\left\|\widetilde{p}_{\mathrm{ex}}^{\mathfrak{Q}}\right\|_{2}^{2} \leq C_{23} \operatorname{size}(\mathcal{T})^{2}\left(\|\mathbf{u}\|_{\left(H^{2}(\mathfrak{Q})\right)^{2}}^{2}+\|p\|_{H^{1}(\mathfrak{Q})}^{2}\right) .
$$

LEMMA 6.5. We assume that $(\mathbf{u}, p)$ is the solution of the problem (1.1). There exists a constant $C_{24}>0$, depending only on $\underline{\mathrm{C}}_{\eta}, \overline{\mathrm{C}}_{\eta}, \mathrm{C}_{\eta}, \operatorname{reg}(\mathcal{T})$ and $\sin \left(\epsilon_{0}\right)$, such that

$$
\begin{aligned}
& m_{\mathcal{D}}\left|\operatorname{div}^{\mathcal{D}} \mathbb{P}_{\boldsymbol{c}}^{\mathcal{T}} \mathbf{u}\right|^{2} \leq\left\|\mathrm{D}_{\mathfrak{Q}}^{\mathcal{N}} \mathbb{P}_{\boldsymbol{c}}^{\mathcal{T}} \mathbf{u}-\mathrm{Du}\right\|_{\mathfrak{Q}_{\mathcal{D}}}^{2}, \quad \forall \mathcal{D} \in \mathfrak{D}, \\
& \left\|\operatorname{div}^{\mathfrak{D}} \mathbb{P}_{\boldsymbol{c}}^{\mathcal{T}} \mathbf{u}\right\|_{2} \leq C_{24} \operatorname{size}(\mathcal{T})\left(\|\mathbf{u}\|_{\left(H^{2}(\mathfrak{Q})\right)^{2}}+\|p\|_{H^{1}(\mathfrak{Q})}\right) .
\end{aligned}
$$

Proof. Thanks to $\operatorname{div}^{\mathcal{D}}\left(\mathbb{P}_{c}^{\mathcal{T}} \mathbf{u}\right)=\operatorname{Tr}\left(\mathrm{D}^{\mathcal{D}} \mathbb{P}_{\boldsymbol{c}}^{\mathcal{T}} \mathbf{u}\right)$ and $\operatorname{div} \mathbf{u}=0$, the equality (2.7) gives

$$
\operatorname{div}^{\mathcal{D}}\left(\mathbb{P}_{\boldsymbol{c}}^{\mathcal{T}} \mathbf{u}\right)=\operatorname{div}^{\mathcal{D}}\left(\mathbb{P}_{\boldsymbol{c}}^{\mathcal{T}} \mathbf{u}\right)-\operatorname{div} \mathbf{u}=\frac{1}{m_{\mathcal{D}}} \sum_{\mathcal{Q} \in \mathfrak{Q}_{\mathcal{D}}} \int_{\mathcal{Q}} \operatorname{Tr}\left(\mathrm{D}_{\mathcal{Q}}^{\mathcal{N}} \mathbb{P}_{\boldsymbol{c}}^{\mathcal{T}} \mathbf{u}-\operatorname{Du}(z)\right) \mathrm{d} z .
$$

Cauchy-Schwarz inequality implies the first estimate. Thanks to (6.19), we get the second estimate with $C_{24}=\sqrt{C_{23}}$.

Now, we can control $\mathbf{R}_{\mathfrak{s}, \mathcal{Q}}^{\mathbf{u}}$, as follows

LEMMA 6.6. We assume that $(\mathbf{u}, p)$ is the solution of the problem (1.1). There exists a constant $C_{25}>0$, depending only on $\mathrm{C}_{\eta}, \underline{\mathrm{C}}_{\eta}, \overline{\mathrm{C}}_{\eta}, \operatorname{reg}(\mathcal{T})$ and $\sin \left(\epsilon_{0}\right)$, such that

$$
\left\|\mathbf{R}_{\mathfrak{s}, \mathfrak{Q}}^{\mathbf{u}}\right\|_{2} \leq C_{25} \operatorname{size}(\mathcal{T})\left(\|\mathbf{u}\|_{\left(H^{2}(\mathfrak{Q})\right)^{2}}+\|p\|_{H^{1}(\mathfrak{Q})}\right) .
$$

Proof. Definition 6.2 implies that

$$
\left\|\mathbf{R}_{\mathfrak{s}, \mathfrak{Q}}^{\mathbf{u}}\right\|_{2}^{2} \leq \sum_{\mathcal{Q} \in \mathfrak{Q}} m_{\mathcal{Q}} \sum_{\mathfrak{s} \in \mathcal{E}_{\mathcal{Q}}}\left|\mathbf{R}_{\mathfrak{s}, \mathcal{Q}}^{\mathbf{u}, \eta}\right|^{2}+\left\|R_{\mathfrak{Q}}^{\mathbf{u}, \mathrm{Du}}\right\|_{2}^{2}
$$

First, the inequality (1.2) and Lemma 6.4 imply

$$
\left\|R_{\mathfrak{Q}}^{\mathbf{u}, \mathrm{Du}}\right\|_{2}^{2} \leq \overline{\mathrm{C}}_{\eta}^{2} C_{23} \operatorname{size}(\mathcal{T})^{2} \sum_{\mathcal{Q} \in \mathfrak{Q}} \int_{\mathcal{Q}}\left(\|\nabla \mathbf{u}(x)\|_{\mathcal{F}}^{2}+\left|\nabla^{2} \mathbf{u}(x)\right|^{2}+|\nabla p|^{2}\right) \mathrm{d} x .
$$

Finally, Lemma 6.3 implies the result, noting $C_{25}=\sqrt{\overline{\mathrm{C}}_{\eta}^{2} C_{23}+C_{20}}$.

6.5. Pressure jumps in diamonds. LEMMA 6.7. We assume that $(\mathbf{u}, p)$ is the solution of the problem (1.1). There exists $C_{26}>0$, depending only on $\underline{\mathrm{C}}_{\eta}, \overline{\mathrm{C}}_{\eta}, \mathrm{C}_{\eta}, \operatorname{reg}(\mathcal{T})$ and $\sin \left(\epsilon_{0}\right)$, such that

$$
\sum_{\mathfrak{s}=\mathcal{Q}\left|\mathcal{Q}^{\prime}=\mathcal{D}\right| \mathcal{D}^{\prime} \in \mathfrak{S}}\left(p_{\mathrm{ex}}^{\mathcal{Q}^{\prime}}-p_{\mathrm{ex}}^{\mathcal{Q}}\right)^{2} \leq C_{26}\left(\|\mathbf{u}\|_{\left(H^{2}(\mathfrak{Q})\right)^{2}}^{2}+\|p\|_{H^{1}(\mathfrak{Q})}^{2}\right)
$$

Proof. We note $\mathbb{P}_{m}^{\mathfrak{s}} p:=\frac{1}{m_{\mathfrak{s}}} \int_{\mathfrak{s}} p(y) \mathrm{d} y$, for any $\mathfrak{s} \in \mathfrak{S}$, adding and subtracting $\mathbb{P}_{m}^{\mathfrak{s}} p$, Cauchy-Schwarz inequality implies

$$
\sum_{\mathfrak{s}=\mathcal{Q}\left|\mathcal{Q}^{\prime}=\mathcal{D}\right| \mathcal{D}^{\prime} \in \mathfrak{S}}\left(p_{\mathrm{ex}}^{\mathcal{Q}^{\prime}}-p_{\mathrm{ex}}^{\mathcal{Q}}\right)^{2} \leq 4 \sum_{\mathcal{D} \in \mathcal{D}} \sum_{\mathcal{Q} \in \mathfrak{Q}_{\mathcal{D}} \in{ }_{\mathfrak{s}} \in \mathcal{Q} \cap \partial \mathcal{D}}\left|p_{\mathrm{ex}}^{\mathcal{Q}}-\mathbb{P}_{m}^{\mathfrak{s}} p\right|^{2} .
$$

Then adding and subtracting $\mathbb{P}_{m}^{\mathcal{O}} p$, Cauchy-Schwarz inequality implies

$$
\sum_{\mathfrak{s}=\mathcal{Q}\left|\mathcal{Q}^{\prime}=\mathcal{D}\right| \mathcal{D}^{\prime} \in \mathfrak{S}}\left(p_{\text {ex }}^{\mathcal{Q}^{\prime}}-p_{\text {ex }}^{\mathcal{Q}}\right)^{2} \leq 8 \sum_{\mathcal{D} \in \mathcal{D}} \sum_{\mathcal{Q} \in \mathfrak{Q}_{\mathcal{D}}}\left|p_{\text {ex }}^{\mathcal{Q}}-\mathbb{P}_{m}^{\mathcal{Q}} p\right|^{2}+8 \sum_{\mathcal{D} \in \mathcal{D}} \sum_{\mathcal{Q} \in \mathfrak{Q}_{\mathcal{D}} \mathfrak{s} \in \partial \mathcal{Q} \cap \partial \mathcal{D}}\left|\mathbb{P}_{m}^{\mathcal{Q}} p-\mathbb{P}_{m}^{\mathfrak{s}} p\right|^{2}
$$


Since we have $p \in H^{1}(\mathcal{Q})$, thanks to Lemma 4.5, we get

$$
\left|\mathbb{P}_{m}^{\mathcal{Q}} p-\mathbb{P}_{m}^{\mathfrak{s}} p\right|^{2} \leq C_{11} \operatorname{reg}(\mathcal{T})^{3} \int_{\mathcal{Q}}|\nabla p(z)|^{2} \mathrm{~d} z .
$$

Lemma 6.4 and (6.21) conclude the proof with $C_{26}=8\left(C_{23}+C_{11} \operatorname{reg}(\mathcal{T})^{3}\right)$.

6.6. End of the proof of Theorem 6.1. We may now collect all the previous results in order to conclude the proof of Theorem 6.1, that we started in Section 6.2.

Proof. Having denoted by $\mathbf{e}^{\mathcal{T}}=\mathbb{P}_{c}^{\mathcal{T}} \mathbf{u}-\mathbf{u}^{\mathcal{T}}$ and $e^{\mathfrak{D}}=\mathbb{P}_{m}^{\mathfrak{D}} p-p^{\mathfrak{D}}$, we have obtained the inequality $(6.8)$

$$
\begin{aligned}
\left\|\nabla_{\mathfrak{Q}}^{\mathcal{N}} \mathbf{e}^{\mathcal{T}}\right\|_{2}^{2}+\left\|e^{\mathfrak{Q}}\right\|_{2}^{2} \leq \widetilde{C}_{1}\left(\left\|\nabla_{\mathfrak{Q}}^{\mathcal{N}} \mathbf{e}^{\mathcal{T}}\right\|_{2}+\left\|e^{\mathfrak{Q}}\right\|_{2}\right)\left(\left\|\mathbf{R}_{\mathfrak{s}, \mathfrak{Q}}\right\|_{2}+\left\|\operatorname{div}^{\mathfrak{D}} \mathbb{P}_{\boldsymbol{c}}^{\mathcal{T}} \mathbf{u}\right\|_{2}\right) \\
+\widetilde{C}_{2} \operatorname{size}(\mathcal{T})\left(\left\|\nabla_{\mathfrak{Q}}^{\mathcal{N}} \mathbf{e}^{\mathcal{T}}\right\|_{2}+\left\|e^{\mathfrak{Q}}\right\|_{2}\right)\left(\sum_{\mathfrak{s}=\mathcal{Q}\left|\mathcal{Q}^{\prime}=\mathcal{D}\right| \mathcal{D}^{\prime} \in \mathfrak{S}}\left(p_{\mathrm{ex}}^{\mathcal{Q}^{\prime}}-p_{\mathrm{ex}}^{\mathcal{Q}}\right)^{2}\right)^{\frac{1}{2}} .
\end{aligned}
$$

Using the estimate $\left\|\mathbf{R}_{\mathfrak{s}, \mathfrak{Q}}\right\|_{2} \leq 2\left\|\mathbf{R}_{\mathfrak{s}, \mathfrak{Q}}^{\mathbf{u}}\right\|_{2}+\left\|\mathbf{R}_{\mathfrak{s}, \mathfrak{Q}}^{p}\right\|_{2}+\left\|\widetilde{p}_{\mathrm{ex}}^{\mathfrak{Q}_{\mathfrak{D}}}\right\|_{2}$, Lemmas 6.1, 6.19, 6.6 and Lemma 6.5 imply $\left\|\mathbf{R}_{\mathfrak{s}, \mathfrak{Q}}\right\|_{2}+\left\|\operatorname{div}^{\mathfrak{D}} \mathbb{P}_{c}^{\mathcal{T}} \mathbf{u}\right\|_{2} \leq C \operatorname{size}(\mathcal{T})$. Finally, Lemma 6.7 gives

$$
\left\|\nabla_{\mathfrak{Q}}^{\mathcal{N}} \mathbf{e}^{\mathcal{T}}\right\|_{2} \leq C \operatorname{size}(\mathcal{T}) \quad \text { and } \quad\left\|e^{\mathfrak{Q}}\right\|_{2} \leq C \operatorname{size}(\mathcal{T}) .
$$

Estimate of $\left\|\mathbf{u}-\mathbf{u}^{\mathcal{T}}\right\|_{2}$. We have $\left\|\mathbf{u}-\mathbf{u}^{\mathcal{T}}\right\|_{2} \leq\left\|\mathbf{u}-\mathbb{P}_{c}^{\mathcal{T}} \mathbf{u}\right\|_{2}+\left\|\mathbb{P}_{c}^{\mathcal{T}} \mathbf{u}-\mathbf{u}^{\mathcal{T}}\right\|_{2}$. Lemma 6.2 and the discrete Poincaré inequality Theorem 4.5 imply

$$
\left\|\mathbf{u}-\mathbf{u}^{\mathcal{T}}\right\|_{2} \leq C \operatorname{size}(\mathcal{T})+C\left\|\nabla^{\mathcal{D}} \mathbb{P}_{c}^{\mathcal{T}} \mathbf{u}-\nabla^{\mathcal{D}} \mathbf{u}^{\mathcal{T}}\right\|_{2} .
$$

Lemma 4.2 and (6.22) gives the estimate of $\left\|\mathbf{u}-\mathbf{u}^{\mathcal{T}}\right\|_{2}$.

Estimate of $\|$ Du- $-\mathrm{D}_{\mathfrak{2}}^{\mathcal{N}} \mathbf{u}^{\mathcal{T}} \|_{2}$. We have $\left\|\mathrm{D} \mathbf{u}-\mathrm{D}_{\mathfrak{2}}^{\mathcal{N}} \mathbf{u}^{\mathcal{T}}\right\|_{2} \leq\left\|\mathrm{Du}-\mathrm{D}_{\mathfrak{2}}^{\mathcal{N}} \mathbb{P}_{c}^{\mathcal{T}} \mathbf{u}\right\|_{2}+\| \mathrm{D}_{\mathfrak{Q}}^{\mathcal{N}} \mathbb{P}_{c}^{\mathcal{T}} \mathbf{u}-$ $\mathrm{D}_{\mathfrak{2}}^{\mathcal{N}} \mathbf{u}^{\mathcal{T}} \|_{2}$. Finally, (6.19) and (6.22) imply the estimate of $\left\|\mathrm{Du}-\mathrm{D}_{\mathfrak{2}}^{\mathcal{N}} \mathbf{u}^{\mathcal{T}}\right\|_{2}$.

Estimate of $\left\|p-p^{\mathfrak{Q}}\right\|_{2}$. Using (6.22), we obtain $\left\|\mathbb{P}_{m}^{\mathfrak{Q}} p-p^{\mathfrak{Q}}\right\|_{2} \leq C \operatorname{size}(\mathcal{T})$. We conclude thanks to Lemma 6.1.

Remark that we can improve the estimate of the velocity as follows

COROLLARY 6.1. If for any $\mathcal{D} \in \mathfrak{D}$, we have $\alpha_{\mathcal{K}} \neq \alpha_{\mathcal{L}}$. We assume that the assumption of Theorem 6.1 are satisfied. There exists a constant $C_{27}>0$ depending only on $\operatorname{reg}(\mathcal{T}), \lambda$, $\sin \left(\epsilon_{0}\right), \mathrm{C}_{\eta}, \underline{\mathrm{C}}_{\eta}, \overline{\mathrm{C}}_{\eta},\|\mathbf{u}\|_{\left(H^{2}(\mathfrak{Q})\right)^{2}}$ and $\|p\|_{H^{1}(\mathfrak{Q})}$, such that:

$$
\left\|\nabla \mathbf{u}-\nabla_{\mathfrak{Q}}^{\mathcal{N}} \mathbf{u}^{\mathcal{T}}\right\|_{2} \leq C_{27} \operatorname{size}(\mathcal{T}) .
$$

Proof. The difficulty lies in the proof of the existence of a constant $C_{28}>0$, such that

$$
\left\|\nabla \mathbf{u}-\nabla_{\mathfrak{Q}}^{\mathcal{N}} \mathbb{P}_{\boldsymbol{c}}^{\mathcal{T}} \mathbf{u}\right\|_{2} \leq C_{28} \operatorname{size}(\mathcal{T})\left(\|\mathbf{u}\|_{\left(H^{2}(\mathfrak{Q})\right)^{2}}+\|p\|_{H^{1}(\mathfrak{Q})}\right) .
$$

Indeed with this estimate, we have

$$
\left\|\nabla \mathbf{u}-\nabla_{\mathfrak{Q}}^{\mathcal{N}} \mathbf{u}^{\mathcal{T}}\right\|_{2} \leq C_{28} \operatorname{size}(\mathcal{T})\left(\|\mathbf{u}\|_{\left(H^{2}(\mathfrak{Q})\right)^{2}}+\|p\|_{H^{1}(\mathfrak{Q})}\right)+\left\|\nabla_{\mathfrak{Q}}^{\mathcal{N}} \mathbb{P}_{c}^{\mathcal{T}} \mathbf{u}-\nabla_{\mathfrak{Q}}^{\mathcal{N}} \mathbf{u}^{\mathcal{T}}\right\|_{2} .
$$

Finally, (6.22) imply the estimate of $\left\|\nabla \mathbf{u}-\nabla_{\mathfrak{Q}}^{\mathcal{N}} \mathbf{u}^{\mathcal{T}}\right\|_{2}$. We prove now the existence of $C_{28}$. Let $\mathcal{D} \in \mathfrak{D}$. Thanks to Proposition 6.1, there exists $\delta_{\text {ex }}^{\mathcal{D}} \in \mathcal{M}_{n_{\mathcal{D}}, 2}(\mathbb{R})$ such that $\left(\delta_{\text {ex }}^{\mathcal{D}}: \delta_{0}\right)=0$ and $\mathrm{D}_{\mathcal{Q}}^{\mathcal{N}} \mathbb{P}_{c}^{\mathcal{T}} \mathbf{u}=\mathrm{D}^{\mathcal{D}} \mathbb{P}_{c}^{\mathcal{T}} \mathbf{u}+\frac{1}{2}\left(B_{\mathcal{Q}} \delta_{\mathrm{ex}}^{\mathcal{D}}+{ }^{t} \delta_{\mathrm{ex}}^{\mathcal{D}}{ }^{t} B_{\mathcal{Q}}\right)$ for all $\mathcal{Q} \in \mathfrak{Q}_{\mathcal{D}}$. So we can write $\nabla_{\mathcal{Q}}^{\mathcal{N}} \mathbb{P}_{c}^{\mathcal{T}} \mathbf{u}=$ $\nabla^{\mathcal{D}} \mathbb{P}_{c}^{\mathcal{T}} \mathbf{u}+{ }^{t} \delta_{\mathrm{ex}}^{\mathcal{D}}{ }^{t} B_{\mathcal{Q}}$ for all $\mathcal{Q} \in \mathfrak{Q}_{\mathcal{D}}$. By the discussion of Section 2.4.1 we remark that, there 
exists $\widetilde{\delta^{\mathcal{D}}} \in \mathcal{M}_{n_{\mathcal{D}}, 2}(\mathbb{R})$ such that $\nabla \mathbb{P}_{c}^{\mathcal{Q}} \mathbf{u}=\nabla^{\mathcal{D}} \mathbb{P}_{\boldsymbol{c}}^{\mathcal{T}} \mathbf{u}+{ }^{t} \widetilde{\delta^{\mathcal{D}} t} B_{\mathcal{Q}}$, using the affine function $\Pi_{\mathcal{D}} \mathbb{P}_{c}^{\mathcal{T}} \mathbf{u}$ (see Fig. 2.4), that is

$$
\begin{array}{rlrl}
\widetilde{\delta_{\mathcal{K}}} & =\mathbf{u}\left(x_{\sigma_{\mathcal{K}}}\right)-\Pi_{\mathcal{D}} \mathbb{P}_{c}^{\mathcal{T}} \mathbf{u}\left(x_{\sigma_{\mathcal{K}}}\right) & \widetilde{\delta_{\mathcal{L}}}=\mathbf{u}\left(x_{\sigma_{\mathcal{L}}}\right)-\Pi_{\mathcal{D}} \mathbb{P}_{c}^{\mathcal{T}} \mathbf{u}\left(x_{\sigma_{\mathcal{L}}}\right) \\
\widetilde{\delta_{\mathcal{K}^{*}}} & =\mathbf{u}\left(x_{\sigma_{\mathcal{K}^{*}}}\right)-\Pi_{\mathcal{D}} \mathbb{P}_{c}^{\mathcal{T}} \mathbf{u}\left(x_{\sigma_{\mathcal{K}^{*}}}\right) & \widetilde{\delta_{\mathcal{L}^{*}}} & =\mathbf{u}\left(x_{\sigma_{\mathcal{L}^{*}}}\right)-\Pi_{\mathcal{D}} \mathbb{P}_{c}^{\mathcal{T}} \mathbf{u}\left(x_{\sigma_{\mathcal{L}^{*}}}\right) .
\end{array}
$$

Noting $\widehat{\delta^{\mathcal{D}}}=\widetilde{\delta^{\mathcal{D}}}-\delta_{\text {ex }}^{\mathcal{D}}$, we deduce that $\nabla \mathbb{P}_{c}^{\mathcal{Q}} \mathbf{u}-\nabla_{\mathcal{Q}}^{\mathcal{N}} \mathbb{P}_{c}^{\mathcal{T}} \mathbf{u}={ }^{t} \widehat{\delta^{\mathcal{D}} t} B_{\mathcal{Q}}$ and $\mathrm{DP}_{c}^{\mathcal{Q}} \mathbf{u}-\mathrm{D}_{\mathcal{Q}}^{\mathcal{N}} \mathbb{P}_{c}^{\mathcal{T}} \mathbf{u}=$ $\frac{1}{2}\left(B_{\mathcal{Q}} \widehat{\delta^{\mathcal{D}}}+{ }^{t} \widehat{\delta^{\mathcal{D}} t} B_{\mathcal{Q}}\right)$.

- Case $\alpha_{\mathcal{K}} \neq \alpha_{\mathcal{L}}$. Since $\delta_{0}$ is zero, we have $\left(\widehat{\delta^{\mathcal{D}}}: \delta_{0}\right)=0$ and Lemma 2.2 implies

$$
\sum_{\mathcal{Q} \in \mathfrak{Q}_{\mathcal{D}}} m_{\mathcal{Q}}\left\|B_{\mathcal{Q}} \widehat{\delta^{\mathcal{D}}}\right\|_{\mathcal{F}}^{2} \leq C_{1} \sum_{\mathcal{Q} \in \mathfrak{Q}_{\mathcal{D}}} m_{\mathcal{Q}}\left\|\mathrm{D} \mathbb{P}_{c}^{\mathcal{Q}} \mathbf{u}-\mathrm{D}_{\mathcal{Q}}^{\mathcal{N}} \mathbb{P}_{c}^{\mathcal{T}} \mathbf{u}\right\|_{\mathcal{F}}^{2}
$$

Lemma 6.4 gives

$$
\sum_{\mathcal{Q} \in \mathfrak{Q}_{\mathcal{D}}} m_{\mathcal{Q}}\left\|B_{\mathcal{Q}} \widehat{\delta^{\mathcal{D}}}\right\|_{\mathcal{F}}^{2} \leq C_{1} C_{23} h_{\mathcal{D}}^{2} \sum_{\mathcal{Q} \in \mathfrak{Q}_{\mathcal{D}}} \int_{\mathcal{Q}}\left(\|\nabla \mathbf{u}(z)\|_{\mathcal{F}}^{2}+\left|\nabla^{2} \mathbf{u}(z)\right|^{2}+|\nabla p(z)|^{2}\right) \mathrm{d} z
$$

Proposition 6.2 and (6.24) conclude the proof in that case.

7. Numerical results. We show here some numerical results obtained on the domain $\Omega=] 0,1{ }^{2}$. Error estimates are given for two different tests with a stabilization coefficient $\lambda=10^{-3}$.

In order to illustrate error estimates, the family of meshes (see Fig. 7.1) are obtained by successive global refinement of the original mesh. We recall that in the theoretical study

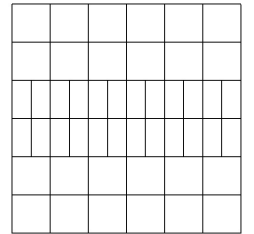

(a) Non conformal quadrangle mesh

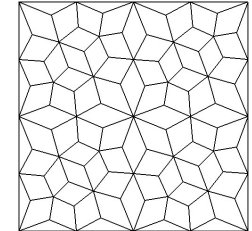

(b) Quadrangle and triangle mesh.

FIG. 7.1. Family of meshes.

presented here, we have either the same angles $\alpha_{\mathcal{K}}$ and $\alpha_{\mathcal{L}}$ (see Fig. 2.2(a)) or the angles distant from $\epsilon_{0}$. This restriction is not required in the numerical test. We get the same results using the barycentric dual mesh or the direct dual mesh. And we observe that the convergence order of the velocity gradient is one even in the case of direct dual mesh.

In all the tests, we choose an exact solution $(\mathbf{u}, p)$ and a viscosity $\eta$ and then define the source term $\mathbf{f}$ and the boundary data $\mathrm{g}$ in such a way that (1.1) is satisfied. In Fig. 7.2 and 7.3, we compare the three following schemes the original DDFV scheme (1.4), the m-DDFV scheme (3.1) and the m-DDFV- $\Delta^{D}$ scheme (3.1) with the old stabilization term $-\lambda h_{\mathfrak{D}}^{2} \Delta^{\mathfrak{D}} p^{\mathfrak{D}}$ instead of $-\lambda h_{\mathfrak{D}}^{2} \Delta^{\mathfrak{D}} p^{\mathfrak{Q}}$. The comparison is performed in term of $L^{2}(\Omega)$-norm for the pressure $\frac{\left\|\mathbb{P}_{c}^{\mathfrak{Q}} p-p^{\mathfrak{Q}}\right\|_{2}}{\left\|\mathbb{P}_{c}^{\mathfrak{Q}} p\right\|_{2}}$, for the velocity gradient $\frac{\left\|\mathbb{P}_{c}^{\mathfrak{Q}} \nabla \mathbf{u}-\nabla_{\mathfrak{Q}}^{\mathcal{N}} \mathbf{u}^{\mathcal{T}}\right\|_{2}}{\left\|\mathbb{P}_{c}^{\mathfrak{Q}} \nabla \mathbf{u}\right\|_{2}}$ and for the velocity $\frac{\left\|\mathbb{P}_{c}^{\mathcal{T}} \mathbf{u}-\mathbf{u}^{\mathcal{T}}\right\|_{2}}{\left\|\mathbb{P}_{\boldsymbol{c}}^{\mathcal{T}} \mathbf{u}\right\|_{2}}$ resp. as a function of the mesh size, in a logarithmic scale, where $\mathbb{P}_{c}^{\mathfrak{Q}} p=\left(\left(p\left(x_{\mathfrak{Q}}\right)\right)_{\mathfrak{Q} \in \mathfrak{Q}}\right)$ is the center-value projection on $\mathfrak{Q}$. 
7.1. Test 1 - Discontinuous viscosity. The interface $\Gamma$ is located at $\{y=0.5\}$. Let $\mathbf{u}(x, y)=\left(\begin{array}{c}u(x, y) \\ 0\end{array}\right)$, with:

$$
u(x, y)=\left\{\begin{array}{rr}
y^{2}-0.5 y & \text { for } y>0.5 \\
10^{4}\left(y^{2}-0.5 y\right) & \text { else. }
\end{array} \quad p(x, y)=2 x-1,\right.
$$

and the discontinuous viscosity: $\eta_{1}=1, \eta_{2}=10^{-4}$, which leads to $\mathrm{D} u$ discontinuous across $\Gamma$. We use the non conformal quadrangle mesh, locally refined where the discontinuity occurs, shown on Fig. 7.1(a).

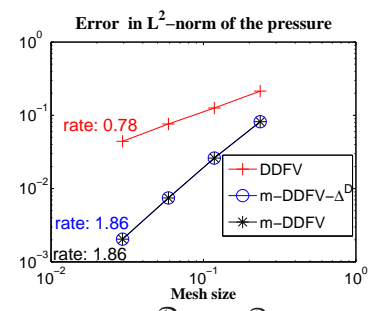

(a) $\frac{\left\|\mathbb{P}_{c}^{\mathfrak{D}} p-p^{\mathfrak{D}}\right\|_{2}}{\left\|\mathbb{P}_{\boldsymbol{c}}^{\mathfrak{D}} p\right\|_{2}}$

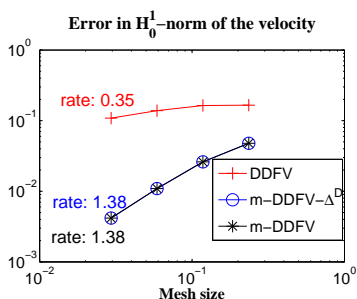

(b) $\frac{\left\|\nabla^{\mathfrak{D}} \mathbb{P}_{c}^{\mathcal{T}} \mathbf{u}-\nabla^{\mathfrak{D}} \mathbf{u}^{\mathcal{T}}\right\|_{2}}{\left\|\nabla^{\mathfrak{D}} \mathbb{P}_{c}^{\mathcal{T}} \mathbf{u}\right\|_{2}}$

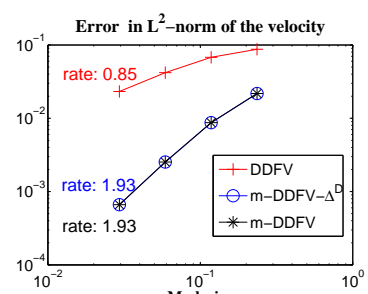

(c) $\frac{\left\|\mathbb{P}_{c}^{\mathcal{T}} \mathbf{u}-\mathbf{u}^{\mathcal{T}}\right\|_{2}}{\left\|\mathbb{P}_{c}^{\mathcal{T}} \mathbf{u}\right\|_{2}}$

FIG. 7.2. Test 1, discontinuous viscosity on a non conformal quadrangle mesh Fig. 7.1(a).

In Fig 7.2, we show that in that case, the results using the m-DDFV- $\Delta^{D}$ scheme are essentially the same than the one using the m-DDFV scheme (3.1). As predicted by the theory, the m-DDFV scheme provides a much better convergence rate than the original DDFV scheme. Furthermore, the error (in any of the three norms we consider) obtained by the mDDFV scheme is better than using the original DDFV scheme even in the case of coarse meshes. Note that the convergence rates obtained with the m-DDFV scheme are greater than the theoretical ones. This is related to some uniformity of the mesh away from the refinement area. Furthermore, let us emphasize that the convergence rate is not sensitive to the presence of non conformal control volumes.

7.2. Test 2 - Discontinuous viscosity and discontinuous pressure. The interface $\Gamma$ is now located at $\{x=0.5\}$. We note $c=-\frac{\eta_{2} \pi}{\eta_{1}+0.5 \eta_{2} \pi}$. We take the discontinuous viscosity: $\eta_{1}=10^{2}, \eta_{2}=10^{-2}$, and

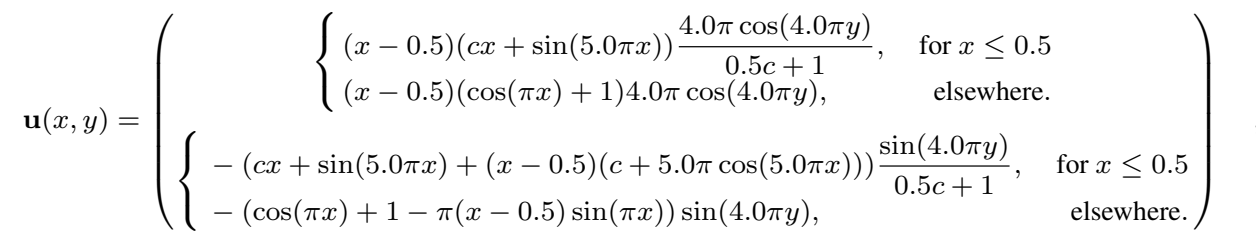

$$
\begin{aligned}
& p(x, y)=\left\{\begin{array}{lr}
8.0 \pi\left(\eta_{1}-\eta_{2}\right) \cos (4 \pi y)+\cos (4 \pi x) \sin (4 \pi y), & \text { for } x \leq 0.5 \\
\cos (4 \pi x) \sin (4 \pi y), & \text { elsewhere. }
\end{array}\right.
\end{aligned}
$$

We use the quadrangle-triangle mesh shown on Fig. 7.1(b). As predicted in Theorem 6.1, we observe for the m-DDFV a first order convergence for the $L^{2}(\Omega)$-norm of the velocity gradient and of the pressure, which seems to be optimal in that case. We obtain a second order convergence for the $L^{2}(\Omega)$-norm of the velocity. This super-convergence of the $L^{2}(\Omega)$ norm is classical for finite volume methods, however its proof in general remains an open 


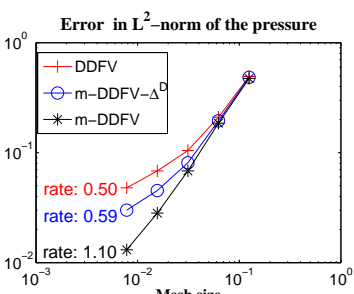

(a) $\frac{\left\|\mathbb{P}_{c}^{\mathfrak{Q}} p-p^{\mathfrak{Q}}\right\|_{2}}{\left\|\mathbb{P}_{c}^{\mathfrak{Q}} p\right\|_{2}}$

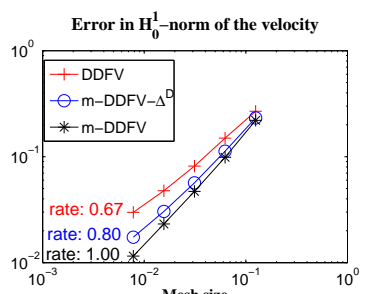

(b) $\frac{\left\|\mathbb{P}_{c}^{\mathfrak{Q}} \nabla \mathbf{u}-\nabla_{\mathfrak{Q}}^{\mathcal{N}} \mathbf{u}^{\mathcal{T}}\right\|_{2}}{\left\|\mathbb{P}_{C}^{\mathfrak{Q}} \nabla \mathbf{u}\right\|_{2}}$

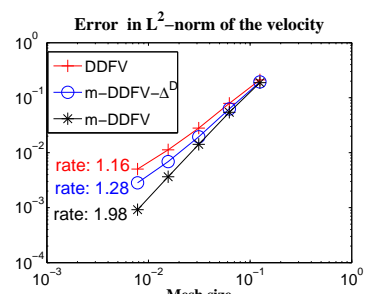

(c) $\frac{\left\|\mathbb{P}_{c}^{\mathcal{T}} \mathbf{u}-\mathbf{u}^{\mathcal{T}}\right\|_{2}}{\left\|\mathbb{P}_{c}^{\mathcal{T}} \mathbf{u}\right\|_{2}}$

FIG. 7.3. Test 2, discontinuous viscosity and discontinuous pressure on the quadrangle-triangle mesh Fig. 7.1(b).

problem (see [26]). Fig. 7.3 brings out the role of the new stabilization term. We observe that the m-DDFV- $\Delta^{D}$ scheme is still convergent even if we have lost the first order convergence, as expected.

8. Conclusion. In this paper, we provide a modification of the stabilized DDFV scheme with Dirichlet boundary conditions for the interface Stokes problem on general 2D grids in order to take into account discontinuities in the viscosity. The m-DDFV scheme we obtained is proved to present a better consistency of the fluxes at the discontinuities. We prove a first order convergence of the DDFV scheme in the $L^{2}(\Omega)$-norm for the velocity gradient, for the velocity and for the pressure. The performance of the scheme is illustrated by numerical results. Let us mention some of the possible extensions of the present work to more general situations. In this paper, we did not allow the viscosity $\eta$ to depend on Du, so the first extension could be to consider this situation with non-Newtonian flows. A second one could be to extend this work to the 3D case.

Acknowledgments The author want to express its gratitude to Franck Boyer and Florence Hubert for their support during the preparation of this work.

\section{Appendix.}

\subsection{Proof of Lemma 2.2.}

Estimate between $\delta^{\mathcal{D}}$ and the symmetric part of $B_{\mathcal{Q}} \delta^{\mathcal{D}}$. Let us explicit the components of $\delta^{\mathcal{D}}$ in the local basis of the diamond cell.

$$
\begin{aligned}
& { }^{t} \delta_{\mathcal{K}}=\mu_{\mathcal{K}} \overrightarrow{\boldsymbol{\tau}}_{\mathcal{K}^{*}, \mathcal{L}^{*}}+\lambda_{\mathcal{K}} \overrightarrow{\mathbf{n}}_{\sigma \mathcal{K}}, \quad{ }^{t} \delta_{\mathcal{L}}=\mu_{\mathcal{L}} \overrightarrow{\boldsymbol{\tau}}_{\mathcal{K}^{*}, \mathcal{L}^{*}}+\lambda_{\mathcal{L}} \overrightarrow{\mathbf{n}}_{\sigma \mathcal{K}}, \\
& { }^{t} \delta_{\mathcal{K}^{*}}=\mu_{\mathcal{K}^{*}} \vec{\tau}_{\sigma_{\mathcal{K}} \mathcal{K}^{*}}+\lambda_{\mathcal{K}^{*}} \overrightarrow{\mathbf{n}}_{\sigma_{\mathcal{K}} \mathcal{K}^{*}}, \quad{ }^{t} \delta_{\mathcal{L}^{*}}=\mu_{\mathcal{L}^{*}} \overrightarrow{\boldsymbol{\tau}}_{\sigma_{\mathcal{K}} \mathcal{K}^{*}}+\lambda_{\mathcal{L}^{*}} \overrightarrow{\mathbf{n}}_{\sigma_{\mathcal{K}} \mathcal{K}^{*}},
\end{aligned}
$$

where $\mu_{\text {. }}, \lambda$. lie in $\mathbb{R}$. Using notation $S_{\mathcal{Q}}=B_{\mathcal{Q}} \delta^{\mathcal{D}}+{ }^{t} \delta^{\mathcal{D} t} B_{\mathcal{Q}}$, for all $\mathcal{Q} \in \mathfrak{Q}_{\mathcal{D}}$, and the fact that ${ }^{t} X B_{\mathcal{Q}} \delta^{\mathcal{D}} X={ }^{t} X^{t} \delta^{D t} B_{\mathcal{Q}} X$ for any $X \in \mathbb{R}^{2}$, the definition of $B_{\mathcal{Q}}$ and the decomposition of $\delta^{\mathcal{D}}$ imply four equations:

$$
\begin{aligned}
& \mu_{\mathcal{K}} m_{\sigma_{\mathcal{K}}}\left(X, \overrightarrow{\boldsymbol{\tau}}_{\mathcal{K}^{*}, \mathcal{L}^{*}}\right)\left(\overrightarrow{\mathbf{n}}_{\sigma_{\mathcal{K}} \mathcal{K}^{*}}, X\right)+\lambda_{\mathcal{K}} m_{\sigma_{\mathcal{K}}}\left(X, \overrightarrow{\mathbf{n}}_{\sigma \mathcal{K}}\right)\left(\overrightarrow{\mathbf{n}}_{\sigma_{\mathcal{K}} \mathcal{K}^{*}}, X\right) \\
& +m_{\sigma_{\mathcal{K}^{*}}} \mu_{\mathcal{K}^{*}}\left(X, \overrightarrow{\boldsymbol{\tau}}_{\sigma_{\mathcal{K}} \mathcal{K}^{*}}\right)\left(\overrightarrow{\mathbf{n}}_{\sigma \mathcal{K}}, X\right)+\lambda_{\mathcal{K}^{*}} m_{\sigma_{\mathcal{K}^{*}}}\left(X, \overrightarrow{\mathbf{n}}_{\boldsymbol{\sigma}_{\mathcal{K}} \mathcal{K}^{*}}\right)\left(\overrightarrow{\mathbf{n}}_{\boldsymbol{\alpha} \mathcal{K}}, X\right) \\
& =\frac{m_{\mathcal{Q}_{\mathcal{K}, \mathcal{K}^{*}}} t}{2} X S_{\mathcal{Q}_{\mathcal{K}, \mathcal{K}^{*}}} X, \\
& -m_{\sigma_{\mathcal{K}}} \mu_{\mathcal{K}}\left(X, \overrightarrow{\boldsymbol{\tau}}_{\mathcal{K}^{*}, \mathcal{L}^{*}}\right)\left(\overrightarrow{\mathbf{n}}_{\sigma_{\mathcal{K}} \mathcal{K}^{*}}, X\right)-\lambda_{\mathcal{K}} m_{\sigma_{\mathcal{K}}}\left(X, \overrightarrow{\mathbf{n}}_{\sigma \mathcal{K}}\right)\left(\overrightarrow{\mathbf{n}}_{\sigma_{\mathcal{K}} \mathcal{K}^{*}}, X\right) \\
& +m_{\sigma_{\mathcal{L}^{*}}} \mu_{\mathcal{L}^{*}}\left(X, \overrightarrow{\boldsymbol{\tau}}_{\sigma_{\mathcal{K}} \mathcal{K}^{*}}\right)\left(\overrightarrow{\mathbf{n}}_{\boldsymbol{\sigma} \mathcal{K}}, X\right)+\lambda_{\mathcal{L}^{*}} m_{\sigma_{\mathcal{L}^{*}}}\left(X, \overrightarrow{\mathbf{n}}_{\sigma_{\mathcal{K}} \mathcal{K}^{*}}\right)\left(\overrightarrow{\mathbf{n}}_{\boldsymbol{\alpha} \mathcal{K}}, X\right)
\end{aligned}
$$

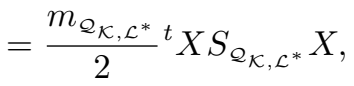




$$
\begin{aligned}
& m_{\sigma_{\mathcal{L}}} \mu_{\mathcal{L}}\left(X, \overrightarrow{\boldsymbol{\tau}}_{\mathcal{K}^{*}, \mathcal{L}^{*}}\right)\left(\overrightarrow{\mathbf{n}}_{\sigma_{\mathcal{L}} \mathcal{K}^{*}}, X\right)+\lambda_{\mathcal{L}} m_{\sigma_{\mathcal{L}}}\left(X, \overrightarrow{\mathbf{n}}_{\sigma \mathcal{K}}\right)\left(\overrightarrow{\mathbf{n}}_{\sigma_{\mathcal{L}} \mathcal{K}^{*}}, X\right) \\
& -m_{\sigma_{\mathcal{K}^{*}}} \mu_{\mathcal{K}^{*}}\left(X, \overrightarrow{\boldsymbol{\tau}}_{\sigma_{\mathcal{K}} \mathcal{K}^{*}}\right)\left(\overrightarrow{\mathbf{n}}_{\sigma \mathcal{K}}, X\right)-\lambda_{\mathcal{K}^{*}} m_{\sigma_{\mathcal{K}^{*}}}\left(X, \overrightarrow{\mathbf{n}}_{\sigma_{\mathcal{K}} \mathcal{K}^{*}}\right)\left(\overrightarrow{\mathbf{n}}_{\sigma \mathcal{K}}, X\right) \\
& =\frac{m_{\mathcal{Q}_{\mathcal{L}, \mathcal{K}^{*}}} t}{2} X S_{\mathcal{Q}_{\mathcal{L}, \mathcal{K}^{*}}} X, \\
& -m_{\sigma_{\mathcal{L}}} \mu_{\mathcal{L}}\left(X, \overrightarrow{\boldsymbol{\tau}}_{\mathcal{K}^{*}, \mathcal{L}^{*}}\right)\left(\overrightarrow{\mathbf{n}}_{\sigma_{\mathcal{L}} \mathcal{K}^{*}}, X\right)-\lambda_{\mathcal{L}} m_{\sigma_{\mathcal{L}}}\left(X, \overrightarrow{\mathbf{n}}_{\sigma \mathcal{K}}\right)\left(\overrightarrow{\mathbf{n}}_{\sigma_{\mathcal{L}} \mathcal{K}^{*}}, X\right) \\
& -m_{\sigma_{\mathcal{L}^{*}}} \mu_{\mathcal{L}^{*}}\left(X, \vec{\tau}_{\sigma_{\mathcal{K}} \mathcal{K}^{*}}\right)\left(\overrightarrow{\mathbf{n}}_{\sigma \mathcal{K}}, X\right)-\lambda_{\mathcal{L}^{*}} m_{\sigma_{\mathcal{L}^{*}}}\left(X, \overrightarrow{\mathbf{n}}_{\sigma_{\mathcal{K}} \mathcal{K}^{*}}\right)\left(\overrightarrow{\mathbf{n}}_{\sigma \mathcal{K}}, X\right) \\
& =\frac{m_{\mathcal{Q}_{\mathcal{L}, \mathcal{L}^{*}}} t}{2} X S_{\mathcal{Q}_{\mathcal{L}, \mathcal{L}^{*}}} X \text {. }
\end{aligned}
$$

We deduce the different value of $\mu$. by taking $X=\vec{\tau}_{\sigma_{\mathcal{K}} \mathcal{K}^{*}}$ in (9.1a)-(9.1b) and by taking $X=\vec{\tau}_{\mathcal{K}^{*}, \mathcal{L}^{*}}$ in (9.1c)-(9.1d). Thanks to the relation (2.2), we have the following estimate

$$
\mu^{2} . \leq C(\operatorname{reg}(\mathcal{T})) \sum_{\mathcal{Q} \in \mathfrak{Q}_{\mathcal{D}}} m_{\mathcal{Q}}\left\|S_{\mathcal{Q}}\right\|_{\mathcal{F}}^{2} .
$$

- Case $\alpha_{\mathcal{K}} \neq \alpha_{\mathcal{L}}$. We have $\left(\overrightarrow{\mathbf{n}}_{\sigma_{\mathcal{L}} \mathcal{K}^{*}}, \overrightarrow{\boldsymbol{\tau}}_{\sigma_{\mathcal{K}} \mathcal{K}^{*}}\right) \neq 0$. We deduce the different value of $\lambda$. by taking in (9.1c) respectively $X=\vec{\tau}_{\sigma_{\mathcal{K}} \mathcal{K}^{*}}$ and $X=\overrightarrow{\boldsymbol{\tau}}_{\sigma_{\mathcal{L}} \mathcal{K}^{*}}$, and in (9.1d) $X=\vec{\tau}_{\sigma_{\mathcal{L}} \mathcal{K}^{*}}$. The value $\lambda_{\mathcal{K}}$ is deduced from (9.1a). Using the criterion $\epsilon_{0}$ and the estimate (9.2), we obtain

$$
\lambda^{2} \leq C\left(\operatorname{reg}(\mathcal{T}), \sin \left(\epsilon_{0}\right)\right) \sum_{\mathcal{Q} \in \mathfrak{Q}_{\mathcal{D}}} m_{\mathcal{Q}}\left\|S_{\mathcal{Q}}\right\|_{\mathcal{F}}^{2} .
$$

Finally, we deduce thanks to (9.2) and (9.3) that

$$
\left\|\delta^{\mathcal{D}}\right\|_{\mathcal{F}}^{2} \leq C \sum_{\mathcal{Q} \in \mathfrak{Q}_{\mathcal{D}}} m_{\mathcal{Q}}\left\|S_{\mathcal{Q}}\right\|_{\mathcal{F}}^{2}=C\left(\operatorname{reg}(\mathcal{T}), \sin \left(\epsilon_{0}\right)\right) \sum_{\mathcal{Q} \in \mathfrak{Q}_{\mathcal{D}}} m_{\mathcal{Q}}\left\|B_{\mathcal{Q}} \delta^{\mathcal{D}}+{ }^{t} \delta^{\mathcal{D} t} B_{\mathcal{Q}}\right\|_{\mathcal{F}}^{2} .
$$

- Case $\alpha_{\mathcal{K}}=\alpha_{\mathcal{L}}$. We have chosen $\delta^{\mathcal{D}}$ such that $\left(\delta^{\mathcal{D}}: \delta_{0}\right)=0$. We write the system on $\lambda$. as follows $B \lambda=F$, where $B$ is a following matrix in $\mathcal{M}_{5,4}(\mathbb{R})$

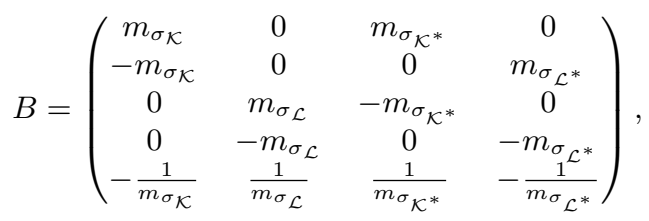

$\lambda={ }^{t}\left(\lambda_{\mathcal{K}}, \lambda_{\mathcal{L}}, \lambda_{\mathcal{K}^{*}}, \lambda_{\mathcal{L}^{*}}\right)$ is a vector in $\mathbb{R}^{4}$ and $F={ }^{t}\left(F_{\mathcal{Q}_{\mathcal{K}, \mathcal{K}^{*}}}, F_{\mathcal{Q}_{\mathcal{K}, \mathcal{L}^{*}}}, F_{\mathcal{Q}_{\mathcal{L}, \mathcal{K}^{*}}}, F_{\mathcal{Q}_{\mathcal{L}, \mathcal{L}^{*}}}, 0\right)$ is a vector in $\mathbb{R}^{5}$. We have $\sum_{\mathcal{Q} \in \mathfrak{Q}_{\mathcal{D}}} F_{\mathcal{Q}}=0$ and using the estimate $(9.2)$, for all $\mathcal{Q} \in \mathfrak{Q}_{\mathcal{D}}$

$$
\left|F_{\mathcal{Q}}\right|^{2} \leq C(\operatorname{reg}(\mathcal{T})) h_{\mathcal{D}}^{2} \sum_{\mathcal{Q} \in \mathfrak{Q}_{\mathcal{D}}} m_{\mathcal{Q}}\left\|S_{\mathcal{Q}}\right\|_{\mathcal{F}}^{2}
$$

The solution of $B \lambda=F$ is

$$
\begin{aligned}
& \lambda_{\mathcal{L}^{*}}=\frac{b}{b^{*}}, \quad \lambda_{\mathcal{K}^{*}}=\frac{1}{m_{\sigma_{\mathcal{K}^{*}}}}\left(F_{\mathcal{Q}_{\mathcal{K}, \mathcal{K}^{*}}}+F_{\mathcal{Q}_{\mathcal{K}, \mathcal{L}^{*}}}-m_{\sigma_{\mathcal{L}^{*}}} \frac{b}{b^{*}}\right), \\
& \lambda_{\mathcal{L}}=\frac{1}{m_{\sigma_{\mathcal{L}}}}\left(-F_{\mathcal{Q}_{\mathcal{L}, \mathcal{L}^{*}}}-m_{\sigma_{\mathcal{L}^{*}}} \frac{b}{b^{*}}\right), \quad \lambda_{\mathcal{K}}=\frac{1}{m_{\sigma_{\mathcal{K}}}}\left(-F_{\mathcal{Q}_{\mathcal{L}, \mathcal{K}^{*}}}+m_{\sigma_{\mathcal{L}^{*}}} \frac{b}{b^{*}}\right),
\end{aligned}
$$

where

$$
|b|^{2} \leq C(\operatorname{reg}(\mathcal{T})) \frac{1}{h_{\mathcal{D}}^{2}} \sum_{\mathcal{Q} \in \mathfrak{Q}_{\mathcal{D}}} m_{\mathcal{Q}}\left\|S_{\mathcal{Q}}\right\|_{\mathcal{F}}^{2} \quad \text { and } \quad\left|b^{*}\right|=-b^{*} \geq C(\operatorname{reg}(\mathcal{T})) \frac{1}{h_{\mathcal{D}}} .
$$


We deduce thanks to (9.5), (9.4) and (9.6) that

$$
\lambda^{2} \leq C(\operatorname{reg}(\mathcal{T})) \sum_{\mathcal{Q} \in \mathfrak{Q}_{\mathcal{D}}} m_{\mathcal{Q}}\left\|S_{\mathcal{Q}}\right\|_{\mathcal{F}}^{2} .
$$

Finally, we deduce thanks to (9.2) and (9.7) that

$$
\left\|\delta^{\mathcal{D}}\right\|_{\mathcal{F}}^{2} \leq C(\operatorname{reg}(\mathcal{T})) \sum_{\mathcal{Q} \in \mathfrak{Q}_{\mathcal{D}}} m_{\mathcal{Q}}\left\|S_{\mathcal{Q}}\right\|_{\mathcal{F}}^{2}=C(\operatorname{reg}(\mathcal{T})) \sum_{\mathcal{Q} \in \mathfrak{Q}_{\mathcal{D}}} m_{\mathcal{Q}}\left\|B_{\mathcal{Q}} \delta^{\mathcal{D}}+{ }^{t} \delta^{\mathcal{D} t} B_{\mathcal{Q}}\right\|_{\mathcal{F}}^{2}
$$

Estimate between $B_{\mathcal{Q}} \delta^{\mathcal{D}}$ and $\delta^{\mathcal{D}}$. Thanks to $h_{\mathcal{D}} \leq C \min \left(m_{\sigma_{\mathcal{K}}}, m_{\sigma_{\mathcal{K}^{*}}}\right)$, we deduce $\left\|B_{\mathcal{Q}} \delta^{\mathcal{D}}\right\|_{\mathcal{F}}^{2} \leq C \frac{1}{h_{\mathcal{D}}^{2}}\left\|\delta^{\mathcal{D}}\right\|_{\mathcal{F}}^{2}$. Thanks to $\operatorname{reg}(\mathcal{T})$, we obtain $\sum_{\mathcal{Q} \in \mathfrak{Q}_{\mathcal{D}}} m_{\mathcal{Q}}\left\|B_{\mathcal{Q}} \delta^{\mathcal{D}}\right\|_{\mathcal{F}}^{2} \leq C\left\|\delta^{\mathcal{D}}\right\|_{\mathcal{F}}^{2}$, that concludes the proof.

9.2. Proof of Lemma 2.3. We improperly note $\alpha_{\mathfrak{s}, \mathcal{D}}\left(\mathrm{D}^{\mathcal{D}} \mathbf{u}^{\mathcal{T}}\right)=\alpha_{\mathcal{Q}}=p^{\mathcal{Q}}-p^{\mathcal{D}}$ when $\mathfrak{s}=\partial \mathcal{D} \cap \partial \mathcal{Q}$. We have that $\left(\delta^{\mathcal{D}}, \alpha_{\mathfrak{Q}_{\mathcal{D}}}\right)$ satisfied the following system

$$
\left\{\begin{array}{l}
\sum_{\mathcal{Q} \in \mathfrak{Q}_{\mathcal{D}}} m_{\mathcal{Q}} \varphi_{\mathcal{Q}}\left(\mathrm{D}^{\mathcal{D}} \mathbf{u}^{\mathcal{T}}, \delta^{\mathcal{D}}, \alpha_{\mathcal{Q}}\right) B_{\mathcal{Q}}=0, \quad \operatorname{Tr}\left({ }^{t} \delta^{\mathcal{D} t} B_{\mathcal{Q}}\right)=0, \forall \mathcal{Q} \in \mathfrak{Q}_{\mathcal{D}}, \\
\sum_{\mathcal{Q} \in \mathfrak{Q}_{\mathcal{D}}} m_{\mathcal{Q}} \alpha_{\mathcal{Q}}=0, \quad\left(\delta^{\mathcal{D}}: \delta_{0}\right)=0 .
\end{array}\right.
$$

Using the value of $\varphi_{\mathcal{Q}}$ in (9.8), we deduce that

$$
\sum_{\mathcal{Q} \in \mathfrak{Q}_{\mathcal{D}}} m_{\mathcal{Q}} \alpha_{\mathcal{Q}} B_{\mathcal{Q}}=2 \sum_{\mathcal{Q} \in \mathfrak{Q}_{\mathcal{D}}} m_{\mathcal{Q}} \eta_{\mathcal{Q}} \mathrm{D}_{\mathcal{Q}}^{\mathcal{N}} \mathbf{u}^{\mathcal{T}} B_{\mathcal{Q}}
$$

We have that $\left\|m_{\mathcal{Q}_{\mathcal{K}, \mathcal{K}^{*}}} B_{\mathcal{Q}_{\mathcal{K}, \mathcal{K}^{*}}}\right\|_{\mathcal{F}}^{2}=m_{\sigma_{\mathcal{K}}}^{2}+m_{\sigma_{\mathcal{K}^{*}}}^{2} \leq 2 h_{\mathcal{D}}^{2}$. The same estimate holds for all $\mathcal{Q} \in \mathfrak{Q}_{\mathcal{D}}$. We estimate the right hand side of (9.9) thanks to Cauchy-Schwarz inequality

$$
\left\|2 \sum_{\mathcal{Q} \in \mathfrak{Q}_{\mathcal{D}}} m_{\mathcal{Q}} \eta_{\mathcal{Q}} \mathrm{D}_{\mathcal{Q}}^{\mathcal{N}} \mathbf{u}^{\mathcal{T}} B_{\mathcal{Q}}\right\|_{\mathcal{F}} \leq 2 \overline{\mathrm{C}}_{\eta} h_{\mathcal{D}}\left(\sum_{\mathcal{Q} \in \mathfrak{Q}_{\mathcal{D}}}\left\|\mathrm{D}_{\mathcal{Q}}^{\mathcal{N}} \mathbf{u}^{\mathcal{T}}\right\|_{\mathcal{F}}^{2}\right)^{\frac{1}{2}}
$$

Then we have that the norm of the left-hand side of (9.9) is

$$
\begin{aligned}
\left\|\sum_{\mathcal{Q} \in \mathfrak{Q}_{\mathcal{D}}} m_{\mathcal{Q}} \alpha_{\mathcal{Q}} B_{\mathcal{Q}}\right\|_{\mathcal{F}}^{2}= & m_{\sigma_{\mathcal{K}}}^{2}\left(\alpha_{\mathcal{Q}_{\mathcal{K}, \mathcal{K}^{*}}}-\alpha_{\mathcal{Q}_{\mathcal{K}, \mathcal{L}^{*}}}\right)^{2}+m_{\sigma_{\mathcal{L}}}^{2}\left(\alpha_{\mathcal{Q}_{\mathcal{L}, \mathcal{K}^{*}}}-\alpha_{\mathcal{Q}_{\mathcal{L}, \mathcal{L}^{*}}}\right)^{2} \\
& +m_{\sigma_{\mathcal{K}^{*}}}^{2}\left(\alpha_{\mathcal{Q}_{\mathcal{K}, \mathcal{K}^{*}}}-\alpha_{\mathcal{Q}_{\mathcal{L}, \mathcal{K}^{*}}}\right)^{2}+m_{\sigma_{\mathcal{L}^{*}}}^{2}\left(\alpha_{\mathcal{Q}_{\mathcal{K}, \mathcal{L}^{*}}}-\alpha_{\mathcal{Q}_{\mathcal{L}, \mathcal{L}^{*}}}\right)^{2}
\end{aligned}
$$

Using (9.10)-(9.11), the relation (2.2) implies that

$$
\left|\alpha_{\mathcal{Q}}-\alpha_{\mathcal{Q}^{\prime}}\right| \leq 2 \overline{\mathrm{C}}_{\eta} \operatorname{reg}(\mathcal{T})\left(\sum_{\mathcal{Q} \in \mathfrak{Q}_{\mathcal{D}}}\left\|\mathrm{D}_{\mathcal{Q}}^{\mathcal{N}} \mathbf{u}^{\mathcal{T}}\right\|_{\mathcal{F}}^{2}\right)^{\frac{1}{2}}, \forall \mathcal{Q}, \mathcal{Q}^{\prime} \in \mathfrak{Q}_{\mathcal{D}} \text { such that } \overline{\mathcal{Q}} \cap \overline{\mathcal{Q}}^{\prime} \neq \emptyset .
$$

Thanks to (4.2) and to $\frac{m_{\mathcal{D}}}{m_{\mathcal{Q}}} \leq \operatorname{reg}(\mathcal{T})^{3}$, we obtain $\sum_{\mathcal{Q} \in \mathfrak{Q}_{\mathcal{D}}}\left\|D_{\mathcal{Q}}^{\mathcal{N}} \mathbf{u}^{\mathcal{T}}\right\|_{\mathcal{F}}^{2} \leq \operatorname{reg}(\mathcal{T})^{3} \frac{\overline{\mathrm{C}}_{\eta}}{\underline{\underline{C}}_{\eta}}\left\|D^{\mathcal{D}} \mathbf{u}^{\mathcal{T}}\right\|_{\mathcal{F}}^{2}$. We deduce that

$$
\left|\alpha_{\mathcal{Q}}-\alpha_{\mathcal{Q}^{\prime}}\right| \leq 2 \overline{\mathrm{C}}_{\eta} \operatorname{reg}(\mathcal{T})^{5 / 2} \frac{\overline{\mathrm{C}}_{\eta}^{1 / 2}}{\underline{\mathrm{C}}_{\eta}^{1 / 2}}\left\|\mathrm{D}^{\mathcal{D}} \mathbf{u}^{\mathcal{T}}\right\|_{\mathcal{F}} .
$$

Now we can estimate $\left|\alpha_{\mathcal{Q}}\right|$ with differences like $\alpha_{\mathcal{Q}^{\prime}}-\alpha_{\mathcal{Q}^{\prime \prime}}$, using (9.8). Thanks to (9.12), we obtain the result with $C_{2}=6 \frac{\operatorname{reg}(\mathcal{T})^{5 / 2} \overline{\mathrm{C}}_{\eta}^{3 / 2}}{\underline{\mathrm{C}}_{\eta}^{1 / 2}}$. 
9.3. Proof of Proposition 6.4. We define $F_{\mathcal{Q}}$ as follows

$$
F_{\mathcal{Q}_{\mathcal{K}, \mathcal{K}^{*}}}=\frac{\mathrm{Id}}{m_{\mathcal{Q}_{\mathcal{K}, \mathcal{K}^{*}}}}, \quad F_{\mathcal{Q}_{\mathcal{K}, \mathcal{L}^{*}}}=-\frac{\mathrm{Id}}{m_{\mathcal{Q}_{\mathcal{K}, \mathcal{L}^{*}}}}, \quad F_{\mathcal{Q}_{\mathcal{L}, \mathcal{K}^{*}}}=-\frac{\mathrm{Id}}{m_{\mathcal{Q}_{\mathcal{L}, \mathcal{K}^{*}}}}, \quad F_{\mathcal{Q}_{\mathcal{K}, \mathcal{L}^{*}}}=\frac{\mathrm{Id}}{m_{\mathcal{Q}_{\mathcal{L}, \mathcal{L}^{*}}}} .
$$

We check that $F_{\mathcal{Q}}$ satisfy the conditions (2.13) and (2.14) if $\alpha_{\mathcal{K}}=\alpha_{\mathcal{L}}$. Thus, there exists $\bar{\delta}^{\mathcal{D}} \in \mathcal{M}_{n_{\mathcal{D}}, 2}(\mathbb{R})$ such that $\left(B_{\mathcal{Q}} \bar{\delta}^{\mathcal{D}}+{ }^{t} \bar{\delta}^{\mathcal{D} t} B_{\mathcal{Q}}\right)=F_{\mathcal{Q}}, \forall \mathcal{Q} \in \mathfrak{Q}_{\mathcal{D}}$ and $\left(\bar{\delta}^{\mathcal{D}}: \delta_{0}\right)=0$. Taking $\delta^{\mathcal{D}}$ equal to $\bar{\delta}^{\mathcal{D}}$ in (6.12), we deduce that

$\sum_{\mathcal{Q} \in \mathfrak{Q}_{\mathcal{D}}} \int_{\mathcal{Q}} \eta(z)\left(F_{\mathcal{Q}}: \operatorname{Du}(z)-\mathrm{D}_{\mathcal{Q}}^{\mathcal{N}} \mathbb{P}_{\boldsymbol{c}}^{\mathcal{T}} \mathbf{u}\right) \mathrm{d} z-\sum_{\mathcal{Q} \in \mathfrak{Q}_{\mathcal{D}}} m_{\mathcal{Q}} \operatorname{Tr}\left(F_{\mathcal{Q}}\right) \widetilde{p}_{\mathrm{ex}}^{\mathcal{Q}}=\operatorname{Tr}\left({ }^{t} \bar{\delta}^{\mathcal{D}} R^{\mathcal{D}}\right)$.

We construct a function $\widetilde{\mathbf{v}}_{\mathcal{D}}$ such that $\widetilde{\mathbf{v}}_{\mathcal{D}} \in\left(H^{1}(\mathcal{D})\right)^{2} \cap\left(H^{2}(\mathcal{Q})\right)^{2}$, for all $\mathcal{Q} \in \mathfrak{Q}_{\mathcal{D}}$, such that

$$
\operatorname{div}\left(\widetilde{\mathbf{v}}_{\mathcal{D}}\right)(x)=\left\{\begin{array}{l}
-\widetilde{p}_{\mathrm{ex}}^{\mathcal{Q}}+\frac{\operatorname{div}^{\mathcal{D}}\left(\mathbb{P}_{\boldsymbol{c}}^{\mathcal{T}} \mathbf{u}\right)}{2 m_{\mathcal{Q}}} \int_{\mathcal{Q}} \eta(z) \mathrm{d} z-\frac{1}{2} \operatorname{Tr}\left({ }^{t} \bar{\delta}^{\mathcal{D}} R^{\mathcal{D}}\right), \text { if } x \in \mathcal{Q}_{\mathcal{K}, \mathcal{K}^{*}}, \\
-\widetilde{p}_{\mathrm{ex}}^{\mathcal{Q}}+\frac{\operatorname{div}^{\mathcal{D}}\left(\mathbb{P}_{\boldsymbol{c}}^{\mathcal{T}} \mathbf{u}\right)}{2 m_{\mathcal{Q}}} \int_{\mathcal{Q}} \eta(z) \mathrm{d} z, \text { if } x \in \mathcal{Q}, \mathcal{Q} \cap \mathcal{Q}_{\mathcal{K}, \mathcal{K}^{*}}=\emptyset,
\end{array}\right.
$$

with $R^{\mathcal{D}}$ defined by Definition 6.4. We choose $\widetilde{\mathbf{v}}_{\mathcal{D}}$ linear per quarter diamond cells of the form $A_{\mathcal{Q}}\left(x-x_{\mathcal{D}}\right)$ if $x \in \mathcal{Q}$, with

$$
\begin{aligned}
& A_{\mathcal{Q}_{\mathcal{K}, \mathcal{K}^{*}}}=\frac{\widetilde{\mathbf{v}}_{\mathcal{K}^{*}}^{\mathcal{D}} \otimes \overrightarrow{\mathbf{n}}_{\sigma_{\mathcal{K}} \mathcal{K}^{*}}}{\overrightarrow{\mathbf{n}}_{\sigma_{\mathcal{K}} \mathcal{K}^{*}} \cdot \overrightarrow{\boldsymbol{\tau}}_{\mathcal{K}^{*}, \mathcal{L}^{*}}}+\frac{\widetilde{\mathbf{v}}_{\mathcal{K}}^{\mathcal{D}} \otimes \overrightarrow{\mathbf{n}}_{\sigma \mathcal{K}}}{\overrightarrow{\mathbf{n}}_{\sigma \mathcal{K}} \cdot \vec{\tau}_{\sigma_{\mathcal{K}^{\mathcal{K}}}}}, \quad A_{\mathcal{Q}_{\mathcal{K}, \mathcal{L}^{*}}}=\frac{\widetilde{\mathbf{v}}_{\mathcal{L}^{*}}^{\mathcal{D}} \otimes \overrightarrow{\mathbf{n}}_{\sigma_{\mathcal{K}} \mathcal{K}^{*}}}{\overrightarrow{\mathbf{n}}_{\sigma_{\mathcal{K}} \mathcal{K}^{*}} \cdot \overrightarrow{\boldsymbol{\tau}}_{\mathcal{K}^{*}, \mathcal{L}^{*}}}+\frac{\widetilde{\mathbf{v}}_{\mathcal{K}}^{\mathcal{D}} \otimes \overrightarrow{\mathbf{n}}_{\sigma \mathcal{K}}}{\overrightarrow{\mathbf{n}}_{\sigma \mathcal{K}} \cdot \vec{\tau}_{\sigma_{\mathcal{K}} \mathcal{K}^{*}}}, \\
& A_{\mathcal{Q}_{\mathcal{L}, \mathcal{K}^{*}}}=\frac{\widetilde{\mathbf{v}}_{\mathcal{K}^{*}}^{\mathcal{D}} \otimes \overrightarrow{\mathbf{n}}_{\sigma_{\mathcal{L}} \mathcal{K}^{*}}}{\overrightarrow{\mathbf{n}}_{\sigma_{\mathcal{L}} \mathcal{K}^{*}} \cdot \overrightarrow{\boldsymbol{\tau}}_{\mathcal{K}^{*}, \mathcal{L}^{*}}}+\frac{\widetilde{\mathbf{v}}_{\mathcal{L}}^{\mathcal{D}} \otimes \overrightarrow{\mathbf{n}}_{\sigma \mathcal{K}}}{\overrightarrow{\mathbf{n}}_{\sigma \mathcal{K}} \cdot \vec{\tau}_{\sigma_{\mathcal{L}} \mathcal{K}^{*}}}, \quad A_{\mathcal{Q}_{\mathcal{L}, \mathcal{L}^{*}}}=\frac{\widetilde{\mathbf{v}}_{\mathcal{L}^{*}}^{\mathcal{D}} \otimes \overrightarrow{\mathbf{n}}_{\sigma_{\mathcal{L}} \mathcal{K}^{*}}}{\overrightarrow{\mathbf{n}}_{\sigma_{\mathcal{L}} \mathcal{K}^{*}} \cdot \vec{\tau}_{\mathcal{K}^{*}, \mathcal{L}^{*}}}+\frac{\widetilde{\mathbf{v}}_{\mathcal{L}}^{\mathcal{D}} \otimes \overrightarrow{\mathbf{n}}_{\sigma \mathcal{K}}}{\overrightarrow{\mathbf{n}}_{\sigma \mathcal{K}} \cdot \vec{\tau}_{\sigma_{\mathcal{L}} \mathcal{K}^{*}}},
\end{aligned}
$$

where $\widetilde{\mathbf{v}}_{\mathcal{K}}^{\mathcal{D}}, \widetilde{\mathbf{v}}_{\mathcal{L}}^{\mathcal{D}}, \widetilde{\mathbf{v}}_{\mathcal{K}^{*}}^{\mathcal{D}}, \widetilde{\mathbf{v}}_{\mathcal{L}^{*}}^{\mathcal{D}}$ belong to $\mathbb{R}^{2}$, they will be determined above. Remark that we have

$$
\widetilde{\mathbf{v}}_{\mathcal{D}}\left(x_{\mathcal{D}}+\alpha \overrightarrow{\boldsymbol{\tau}}_{\mathcal{K}^{*}, \mathcal{L}^{*}}\right)= \begin{cases}\alpha \widetilde{\mathbf{v}}_{\mathcal{K}^{*}}^{\mathcal{D}}, & \text { if } x_{\mathcal{D}}+\alpha \overrightarrow{\boldsymbol{\tau}}_{\mathcal{K}^{*}, \mathcal{L}^{*}} \in \sigma_{\mathcal{K}^{*}}, \\ \alpha \widetilde{\mathbf{v}}_{\mathcal{L}^{*}}^{\mathcal{D}}, & \text { if } x_{\mathcal{D}}+\alpha \overrightarrow{\boldsymbol{\tau}}_{\mathcal{K}^{*}, \mathcal{L}^{*}} \in \sigma_{\mathcal{L}^{*}}\end{cases}
$$

Thus $\widetilde{\mathbf{v}}_{\mathcal{D}}$ is continuous across the diagonals of $\mathcal{D}$ and $\widetilde{\mathbf{v}}_{\mathcal{D}}\left(x_{\mathcal{D}}\right)=0$.

- Case $\alpha_{\mathcal{K}}=\alpha_{\mathcal{L}}$ : We choose $\widetilde{\mathbf{v}}_{\mathcal{K}}^{\mathcal{D}}=a_{\mathcal{K}} \overrightarrow{\mathbf{n}}_{\sigma \mathcal{K}}, \widetilde{\mathbf{v}}_{\mathcal{L}}^{\mathcal{D}}=a_{\mathcal{L}} \overrightarrow{\mathbf{n}}_{\sigma \mathcal{K}}, \widetilde{\mathbf{v}}_{\mathcal{K}^{*}}^{\mathcal{D}}=a_{\mathcal{K}^{*}} \overrightarrow{\boldsymbol{\tau}}_{\mathcal{K}, \mathcal{L}}, \widetilde{\mathbf{v}}_{\mathcal{L}^{*}}^{\mathcal{D}}=$ $a_{\mathcal{L}^{*}} \overrightarrow{\mathbf{n}}_{\sigma^{*} \mathcal{K}^{*}}$. To determine the unknowns $a_{\mathcal{K}}, a_{\mathcal{L}}, a_{\mathcal{K}^{*}}, a_{\mathcal{L}^{*}}$ we impose (9.14):

$$
\begin{aligned}
& a_{\mathcal{K}}=\sin \left(\alpha_{\mathcal{D}}\right)\left(-\widetilde{p}_{\mathcal{Q}_{\mathcal{K}, \mathcal{K}^{*}}}^{\mathrm{ex}}+\frac{\operatorname{div}^{\mathcal{D}}\left(\mathbb{P}_{c}^{\mathcal{T}} \mathbf{u}\right)}{2 m_{\mathcal{Q}_{\mathcal{K}, \mathcal{K}^{*}}}} \int_{\mathcal{Q}_{\mathcal{K}, \mathcal{K}^{*}}} \eta(z) \mathrm{d} z-\frac{1}{2} \operatorname{Tr}\left({ }^{t} \bar{\delta}^{\mathcal{D}} R^{\mathcal{D}}\right)\right):=\sin \left(\alpha_{\mathcal{D}}\right) b_{1}, \\
& a_{\mathcal{L}}=\sin \left(\alpha_{\mathcal{D}}\right)\left(-\widetilde{p}_{\mathcal{Q}_{\mathcal{L}, \mathcal{K}^{*}}}^{\mathrm{ex}}+\frac{\operatorname{div}^{\mathcal{D}}\left(\mathbb{P}_{c}^{\mathcal{T}} \mathbf{u}\right)}{2 m_{\mathcal{Q}_{\mathcal{L}, \mathcal{K}^{*}}}} \int_{\mathcal{Q}_{\mathcal{L}, \mathcal{K}^{*}}} \eta(z) \mathrm{d} z\right):=\sin \left(\alpha_{\mathcal{D}}\right) b_{2}, \\
& a_{\mathcal{K}}+a_{\mathcal{L}^{*}}=\sin \left(\alpha_{\mathcal{D}}\right)\left(-\widetilde{p}_{\mathcal{Q}_{\mathcal{K}, \mathcal{L}^{*}}}^{\mathrm{ex}}+\frac{\operatorname{div}^{\mathcal{D}}\left(\mathbb{P}_{\boldsymbol{c}}^{\mathcal{T}} \mathbf{u}\right)}{2 m_{\mathcal{Q}_{\mathcal{K}, \mathcal{L}^{*}}}} \int_{\mathcal{Q}_{\mathcal{K}, \mathcal{L}^{*}}} \eta(z) \mathrm{d} z\right):=\sin \left(\alpha_{\mathcal{D}}\right) b_{3}, \\
& a_{\mathcal{L}}+a_{\mathcal{L}^{*}}=\sin \left(\alpha_{\mathcal{D}}\right)\left(-\widetilde{p}_{\mathcal{Q}_{\mathcal{L}, \mathcal{L}^{*}}}^{\mathrm{ex}}+\frac{\operatorname{div}^{\mathcal{D}}\left(\mathbb{P}_{c}^{\mathcal{T}} \mathbf{u}\right)}{2 m_{\mathcal{Q}_{\mathcal{L}, \mathcal{L}^{*}}}} \int_{\mathcal{Q}_{\mathcal{L}, \mathcal{L}^{*}}} \eta(z) \mathrm{d} z\right):=\sin \left(\alpha_{\mathcal{D}}\right) b_{4} .
\end{aligned}
$$

We get

$$
a_{\mathcal{K}}=\sin \left(\alpha_{\mathcal{D}}\right) b_{1}, \quad a_{\mathcal{L}}=\sin \left(\alpha_{\mathcal{D}}\right) b_{2}, \quad a_{\mathcal{L}^{*}}=\sin \left(\alpha_{\mathcal{D}}\right)\left(b_{3}-b_{1}\right) .
$$


Thanks to (9.13) that is $b_{1}-b_{2}-b_{3}+b_{4}=0$, we also have $a_{\mathcal{L}}=\sin \left(\alpha_{\mathcal{D}}\right)\left(b_{1}-b_{3}+b_{4}\right)$ and we choose $a_{\mathcal{K}^{*}}=0$. We deduce that there exists $C>0$ depending only on $\operatorname{reg}(\mathcal{T})$ and $\overline{\mathrm{C}}_{\eta}$ such that

$$
\sum_{\mathcal{Q} \in \mathfrak{Q}_{\mathcal{D}}} m_{\mathcal{Q}}\left\|A_{\mathcal{Q}}\right\|_{\mathcal{F}}^{2} \leq C\left(\sum_{\mathcal{Q} \in \mathfrak{Q}_{\mathcal{D}}} m_{\mathcal{Q}}\left|\widetilde{p}_{\text {ex }}^{\mathcal{Q}}\right|^{2}+m_{\mathcal{D}}\left|\operatorname{div}^{\mathcal{D}}\left(\mathbb{P}_{\boldsymbol{c}}^{\mathcal{T}} \mathbf{u}\right)\right|^{2}+m_{\mathcal{D}}\left|\operatorname{Tr}\left({ }^{t} \bar{\delta}^{\mathcal{D}} R^{\mathcal{D}}\right)\right|^{2}\right) .
$$

- Case $\alpha_{\mathcal{K}} \neq \alpha_{\mathcal{L}}$ : We choose $\widetilde{\mathbf{v}}_{\mathcal{K}}^{\mathcal{D}}=a_{\mathcal{K}} \overrightarrow{\mathbf{n}}_{\sigma \mathcal{K}}, \widetilde{\mathbf{v}}_{\mathcal{L}}^{\mathcal{D}}=a_{\mathcal{L}} \overrightarrow{\mathbf{n}}_{\sigma \mathcal{K}}, \widetilde{\mathbf{v}}_{\mathcal{K}^{*}}^{\mathcal{D}}=a_{\mathcal{K}^{*}} \overrightarrow{\boldsymbol{\tau}}_{\sigma_{\mathcal{K}^{\mathcal{K}}}}$, $\widetilde{\mathbf{v}}_{\mathcal{L}^{*}}^{\mathcal{D}}=a_{\mathcal{L}^{*}} \overrightarrow{\mathbf{n}}_{\sigma_{\mathcal{K}} \mathcal{K}^{*}}$. To determine the unknowns $a_{\mathcal{K}}, a_{\mathcal{L}}, a_{\mathcal{K}^{*}}, a_{\mathcal{L}^{*}}$ we impose $(9.14)$ :

$$
\begin{aligned}
& a_{\mathcal{K}}=\sin \left(\alpha_{\mathcal{K}}\right) b_{1}, \quad a_{\mathcal{K}}+a_{\mathcal{L}^{*}}=\sin \left(\alpha_{\mathcal{K}}\right) b_{3}, \\
& a_{\mathcal{L}}+a_{\mathcal{K}^{*}} \sin \left(\alpha_{\mathcal{K}}-\alpha_{\mathcal{L}}\right)=\sin \left(\alpha_{\mathcal{L}}\right) b_{2}, \quad a_{\mathcal{L}}+a_{\mathcal{L}^{*}} \cos \left(\alpha_{\mathcal{K}}-\alpha_{\mathcal{L}}\right)=\sin \left(\alpha_{\mathcal{L}}\right) b_{4} .
\end{aligned}
$$

We get

$$
\begin{aligned}
& a_{\mathcal{K}}=\sin \left(\alpha_{\mathcal{K}}\right) b_{1}, \quad a_{\mathcal{L}}=\sin \left(\alpha_{\mathcal{L}}\right) b_{4}+\cos \left(\alpha_{\mathcal{K}}-\alpha_{\mathcal{L}}\right) \sin \left(\alpha_{\mathcal{K}}\right)\left(b_{1}-b_{3}\right), \\
& a_{\mathcal{K}^{*}}=\frac{\sin \left(\alpha_{\mathcal{L}}\right)\left(b_{2}-b_{4}\right)+\cos \left(\alpha_{\mathcal{K}}-\alpha_{\mathcal{L}}\right) \sin \left(\alpha_{\mathcal{K}}\right)\left(b_{3}-b_{1}\right)}{\sin \left(\alpha_{\mathcal{K}}-\alpha_{\mathcal{L}}\right)}, \quad a_{\mathcal{L}^{*}}=\sin \left(\alpha_{\mathcal{K}}\right)\left(b_{3}-b_{1}\right) .
\end{aligned}
$$

In that case, we have that $a_{\mathcal{K}^{*}}$ blows up if the angles $\alpha_{\mathcal{K}}, \alpha_{\mathcal{L}}$ are too close. So there exists $C>0$ depending only on $\operatorname{reg}(\mathcal{T}), \overline{\mathrm{C}}_{\eta}$ and $\sin \left(\epsilon_{0}\right)$ such that

$$
\sum_{\mathcal{Q} \in \mathfrak{Q}_{\mathcal{D}}} m_{\mathcal{Q}}\left\|A_{\mathcal{Q}}\right\|_{\mathcal{F}}^{2} \leq C\left(\sum_{\mathcal{Q} \in \mathfrak{Q}_{\mathcal{D}}} m_{\mathcal{Q}}\left|\widetilde{p}_{\text {ex }}^{\mathcal{Q}}\right|^{2}+m_{\mathcal{D}}\left|\operatorname{div}^{\mathcal{D}}\left(\mathbb{P}_{\boldsymbol{c}}^{\mathcal{T}} \mathbf{u}\right)\right|^{2}+m_{\mathcal{D}}\left|\operatorname{Tr}\left({ }^{t} \bar{\delta}^{\mathcal{D}} R^{\mathcal{D}}\right)\right|^{2}\right)
$$

From (9.15) and (9.16) and applying Lemma 6.5, we obtain

$$
\left\|\widetilde{\mathbf{v}}_{\mathcal{D}}\right\|_{H^{1}(\mathcal{D})} \leq C\left(\left\|\widetilde{p}_{\mathrm{ex}}^{\mathfrak{Q}_{\mathcal{D}}}\right\|_{2}+\left\|\mathrm{D} \mathbf{u}-\mathrm{D}_{\mathcal{Q}}^{\mathcal{N}} \mathbb{P}_{\boldsymbol{c}}^{\mathcal{T}} \mathbf{u}\right\|_{\mathfrak{Q}_{\mathcal{D}}}+\sqrt{m_{\mathcal{D}}}\left|\operatorname{Tr}\left({ }^{t} \bar{\delta}^{\mathcal{D}} R^{\mathcal{D}}\right)\right|\right) .
$$

Lemma 2.2 implies that $m_{\mathcal{D}}\left\|B_{\mathcal{Q}} \bar{\delta}^{\mathcal{D}}\right\|_{\mathfrak{Q}_{\mathcal{D}}}^{2} \leq C(\operatorname{reg}(\mathcal{T}))$. Thanks to Proposition 6.3, we deduce

$$
m_{\mathcal{D}}\left|\operatorname{Tr}\left({ }^{t} \bar{\delta}^{\mathcal{D}} R^{\mathcal{D}}\right)\right|^{2} \leq C h_{\mathcal{D}}^{2} \sum_{\mathcal{Q} \in \mathfrak{Q}_{\mathcal{D}}} \int_{\mathcal{Q}}\left(\|\nabla \mathbf{u}(z)\|_{\mathcal{F}}^{2}+\left|\nabla^{2} \mathbf{u}(z)\right|^{2}+|\nabla p(z)|^{2}\right) \mathrm{d} z .
$$

We have $\left\|\mathrm{D} \widetilde{\mathbf{v}}_{\mathcal{D}}-\mathrm{D}_{\mathfrak{Q}}^{\mathcal{N}} \mathbb{P}_{\boldsymbol{c}}^{\mathcal{T}} \widetilde{\mathbf{v}}_{\mathcal{D}}\right\|_{\mathfrak{Q}_{\mathcal{D}}} \leq\left\|\widetilde{\mathbf{v}}_{\mathcal{D}}\right\|_{H^{1}(\mathcal{D})}+\left\|\mathrm{D}_{\mathfrak{Q}}^{\mathcal{N}} \mathbb{P}_{\boldsymbol{c}}^{\mathcal{T}} \widetilde{\mathbf{v}}_{\mathcal{D}}\right\|_{\mathfrak{Q}_{\mathcal{D}}}$. Proposition 4.1 and Lemma 6.2 give $\left\|\mathrm{D} \widetilde{\mathbf{v}}_{\mathcal{D}}-\mathrm{D}_{\mathfrak{Q}}^{\mathcal{N}} \mathbb{P}_{\boldsymbol{c}}^{\mathcal{T}} \widetilde{\mathbf{v}}_{\mathcal{D}}\right\|_{\mathfrak{Q}_{\mathcal{D}}} \leq\left(1+C_{18}\right)\left\|\widetilde{\mathbf{v}}_{\mathcal{D}}\right\|_{H^{1}(\mathcal{D})}$. Thanks to (9.17) and (9.18), we deduce that

$$
\left\|\mathrm{D} \widetilde{\mathbf{v}}_{\mathcal{D}}-\mathrm{D}_{\mathfrak{Q}}^{\mathcal{N}} \mathbb{P}_{\boldsymbol{c}}^{\mathcal{T}} \widetilde{\mathbf{v}}_{\mathcal{D}}\right\|_{\mathfrak{Q}_{\mathcal{D}}} \leq C\left(\left\|\widetilde{p}_{\mathrm{ex}}^{\mathfrak{Q}_{\mathcal{D}}}\right\|_{2}+\left\|\mathrm{D} \mathbf{u}-\mathrm{D}_{\mathcal{Q}}^{\mathcal{N}} \mathbb{P}_{\boldsymbol{c}}^{\mathcal{T}} \mathbf{u}\right\|_{\mathfrak{Q}_{\mathcal{D}}}+C h_{\mathcal{D}}\right)
$$

Furthermore, we have

$-\sum_{\mathcal{Q} \in \mathfrak{Q}_{\mathcal{D}}} \int_{\mathcal{Q}} \operatorname{div} \widetilde{\mathbf{v}}_{\mathcal{D}} \widetilde{p}_{\text {ex }}^{\mathcal{Q}} \mathrm{d} z \geq\left\|\widetilde{p}_{\text {ex }}^{\mathcal{Q}_{\mathcal{D}}}\right\|_{2}^{2}-\operatorname{div}^{\mathcal{D}} \mathbb{P}_{\boldsymbol{c}}^{\mathcal{T}} \mathbf{u} \sum_{\mathcal{Q} \in \mathfrak{Q}_{\mathcal{D}}} \int_{\mathcal{Q}} \eta(z) \mathrm{d} z \widetilde{p}_{\text {ex }}^{\mathcal{Q}}-m_{\mathcal{Q}_{\mathcal{K}, \mathcal{K}^{*}}} \operatorname{Tr}\left({ }^{t} \bar{\delta}^{\mathcal{D}} R^{\mathcal{D}}\right) \widetilde{p}_{\mathcal{Q}_{\mathcal{K}, \mathcal{K}^{*}}}^{\text {ex }}$.

Thanks to divu $=0$, Proposition 6.3 gives

$$
-\sum_{\mathcal{Q} \in \mathfrak{Q}_{\mathcal{D}}} \int_{\mathcal{Q}} \operatorname{div} \widetilde{\mathbf{v}}_{\mathcal{D}} \widetilde{p}_{\text {ex }}^{\mathcal{Q}} \mathrm{d} z \geq\left\|\widetilde{p}_{\text {ex }}^{\mathfrak{Q}_{\mathcal{D}}}\right\|_{2}^{2}-C\left\|\mathrm{Du}-\mathrm{D}_{\mathcal{Q}}^{\mathcal{N}} \mathbb{P}_{c}^{\mathcal{T}} \mathbf{u}\right\|_{\mathfrak{Q}_{\mathcal{D}}}\left\|\widetilde{p}_{\text {ex }}^{\mathfrak{Q} \mathcal{D}}\right\|_{2}-C \operatorname{size}(\mathcal{T})\left\|\widetilde{p}_{\text {ex }}^{\mathfrak{Q}_{\mathcal{D}}}\right\|_{2} .
$$

Young inequality concludes the proof.

\section{REFERENCES}

[1] B. Andreianov, F. Boyer, and F. Hubert. Discrete duality finite volume schemes for Leray-Lions type elliptic problems on general 2D-meshes. Num. Meth. for PDEs, 23(1):145-195, 2007. 
[2] L. Beirão da Veiga, V. Gyrya, K. Lipnikov, and G. Manzini. Mimetic finite difference method for the Stokes problem on polygonal meshes. J. Comput. Phys., 228(19):7215-7232, 2009.

[3] L. Beirão da Veiga and K. Lipnikov. A mimetic discretization of the Stokes problem with selected edge bubbles. SIAM J Sci. Comp., 32(2):875-893, 2010.

[4] F. Boyer and P. Fabrie. Eléments d'analyse pour l'étude de quelques modèles d'écoulements de fluides visqueux incompressibles, volume 52 of Mathématiques \& Applications (Berlin) [Mathematics \& Applications]. Springer-Verlag, Berlin, 2006.

[5] F. Boyer and F. Hubert. Finite volume method for $2 \mathrm{~d}$ linear and nonlinear elliptic problems with discontinuities. SIAM J. Numer. Anal., 46(6):3032-3070, 2008.

[6] F. Brezzi and M. Fortin. Mixed and hybrid finite element methods, volume 15 of Springer Series in Computational Mathematics. Springer-Verlag, New York, 1991.

[7] F. Brezzi and J. Pitkäranta. On the stabilization of finite element approximations of the Stokes equations. In Efficient solutions of elliptic systems (Kiel, 1984), volume 10 of Notes Numer. Fluid Mech., pages 11-19. Vieweg, Braunschweig, 1984.

[8] Y. Coudière, J.-P. Vila, and P. Villedieu. Convergence rate of a finite volume scheme for a two-dimensional convection-diffusion problem. M2AN Math. Model. Numer. Anal., 33(3):493-516, 1999.

[9] S. Delcourte. Développement de méthodes de volumes finis pour la mécanique des fluides. PhD thesis, http://tel.archives-ouvertes.fr/tel-00200833/fr/, Université Paul Sabatier, Toulouse, France, 2007.

[10] K. Domelevo and P. Omnes. A finite volume method for the Laplace equation on almost arbitrary twodimensional grids. M2AN Math. Model. Numer. Anal., 39(6):1203-1249, 2005.

[11] J. Droniou and R. Eymard. A mixed finite volume scheme for anisotropic diffusion problems on any grid. Numer. Math., 105(1):35-71, 2006.

[12] J. Droniou and R. Eymard. Study of the mixed finite volume method for Stokes and Navier-Stokes equations. Numerical Methods for Partial Differential Equations, 25(1):137-171, 2009.

[13] R. Eymard, T. Gallouët, and R. Herbin. Finite volume methods. In Ph Ciarlet and J.L. Lions, editors, Handbook of numerical analysis, Vol. VII, Handb. Numer. Anal., VII, pages 715-1022. North-Holland, Amsterdam, 2000.

[14] R. Eymard, T. Gallouët, and R. Herbin. A cell-centered finite-volume approximation for anisotropic diffusion operators on unstructured meshes in any space dimension. IMA J. Numer. Anal., 26(2):326-353, 2006.

[15] R. Eymard, R. Herbin, and J.-C. Latché. On a stabilized colocated finite volume scheme for the Stokes problem. M2AN Math. Model. Numer. Anal., 40(3):501-527, 2006.

[16] V. Girault and P.-A. Raviart. Finite element methods for Navier-Stokes equations, volume 5 of Springer Series in Computational Mathematics. Springer-Verlag, Berlin, 1986. Theory and algorithms.

[17] F. Harlow and J. Welch. Numerical calculation of time-dependent viscous incompressible flow of fluid with free surface. The physics of fluids, 8(12):2182-2189, 1965.

[18] R. Herbin and F. Hubert. Benchmark on discretization schemes for anisotropic diffsion problems on general grids. In R. Eymard and J. M. Herard, editors, Proceedings of Finite Volumes for Complex Applications $V$, Aussois, France, 2008. Wiley.

[19] F. Hermeline. A finite volume method for the approximation of diffusion operators on distorted meshes. $J$. Comput. Phys., 160(2):481-499, 2000.

[20] K. Ito and Z. Li. Interface conditions for Stokes equations with a discontinuous viscosity and surface sources. Appl. Math. Lett., 19(3):229-234, 2006.

[21] S. Krell. Stabilized DDFV schemes for Stokes problem with variable viscosity on general 2d meshes. 2010. to appear in Num. Meth. for PDEs. Available online at http://dx.doi.org/10.1002/num.20603.

[22] S. Krell and G. Manzini. The Discrete Duality Finite Volume method for the Stokes equation on 3D polyhedral meshes. 2010. Preprint. Available online at http://hal.archives-ouvertes.fr/hal-00448465/fr/.

[23] R. A. Nicolaides. Analysis and convergence of the MAC scheme. I. The linear problem. SIAM J. Numer. Anal., 29(6):1579-1591, 1992.

[24] K.i Ohmori and N. Saito. On the convergence of finite element solutions to the interface problem for the Stokes system. J. Comput. Appl. Math., 198(1):116-128, 2007.

[25] M. A. Olshanskii and A. Reusken. Analysis of a Stokes interface problem. Numer. Math., 103(1):129-149, 2006.

[26] P. Omnes. On the second-order convergence of finite volume methods for the Laplace equation on DelaunayVoronoi meshes. 2010. Preprint, Available online at http://hal-cea.archives-ouvertes.fr/cea-00430941/fr/.

[27] R. Temam. Navier-Stokes equations. Theory and numerical analysis. North-Holland Publishing Co., Amsterdam, 1977. Studies in Mathematics and its Applications, Vol. 2. 